\title{
Vokalharmonie und Vokalbestand im Südostjakischen ${ }^{1}$
}

1. Bei der Bearbeitung von KaRJaLAINEns südostjakischen Texten, als ich monatelang Texte aus ein und derselben Mundart vor mir hatte, musste mir unbedingt auffallen, dass es in diesen eine Vokalharmonie, sogar eine ziemlich strenge, gibt, und nicht nur einige Überreste hie und da darauf hinweisen, wie es seit Castrín bis zum heutigen Tag allgemein angenommen wurde (einschlägige Literatur s. FaLUdi, PaisEmlk. 607-8). Alle fünf Südmundarten, aus denen wir über Texte verfügen (d. h.: DN., Ts., Sav., Sotn., Kr.), haben eine Vokalharmonie, ihre Regeln sind überall völlig klar, kleinere Abweichungen zwischen den Regeln der einzelnen Lokalmundarten können jedoch festgestellt werden.

Es gibt dennoch in allen Texten Wörter mit Suffixen, ja sogar ohne Suffixe, in denen palatale und auch velare Vokale vorkommen, die Zahl dieser Wörter mit „gemischtem Vokalismus» ist aber ziemlich klein, und eine gewisse Regelmässigkeit ist auch in diesen zu finden.

Ich habe die Texte aus allen fünf Mundarten auf die Vokalharmonie gründlich und systematisch untersucht. Die Ergebnisse aus vier Mundarten will ich nun zusammengefasst mitteilen. Das Resultat aus der fünften, aus der Sotn.-Mundart steht in vollem Einklang mit dem aus den übrigen, da aber KarJalainen aus der Sotn.-Mundart nur zwei kurze Lieder, insgesamt eine Seite, aufgezeichnet hat, lohnt es sich nicht, die tabellarisch zusammengefassten Ergebnisse aus allen fünf Mundarten mitzuteilen.

Die suffixlosen Wörter sind entweder vom Anfang bis zilm

1 Gekürzt vorgetragen in Helsinki, am 1. Dez. 1967. 
Ende palatal (tillş̆ 'Mond, Monat', ădoaki 'Schneesturm') oder velar ( $\chi u l \hat{\partial} \chi$ 'Rabe', pōyâr 'Insel'); wenn nicht, dann sind es entweder Fremdwörter (DN. iàşsik < ящик, Kr. arş̌in < аршин usw.) oder zusammengesetzte Wörter (ap ăa $\chi$ 'Bruder' = 'Vaters-Sohn', Kr. òroims 'Dienerin' = 'Dienerin-Frau', DN., Ts., Kr. tèdặt 'Speise' = 'zu essendes, essbares Ding' usw.).

Bei allen Suffixen in der Deklination, in der Konjugation, bei den Possessiv- und Ableitungssuffixen gibt es palatal velare Suffixpaare; die Suffixe richten sich nach dem Grundwort bzw. bei Komposita oder Fremdwörtern nach der letzten Silbe des Wortes, z. B.: DN., 'Ts., Sav., Kr. may 'Land', yot

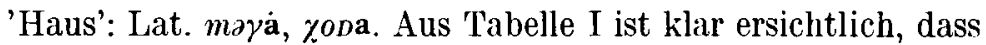
die Lat.-, Instr.-Komit.-, Abess.-, Dual- und Plural-Suffixe je nach dem Grundwort palatal oder velar sind. Auch beim Ablativ gibt es in jeder Mundart palatale und velare Suffixpaare, eine strenge und folgerichtige Vokalharmonie bei dem Ablativsuffix gibt es ja doch nur in der DN.-Yundart; aus Kr. gibt es viele Beispiele, wo velaren Wörtern ein velares Abl.Suffix angehängt wird, aber nach vielen folgt ein palatales, aus Ts. verfügen wir nur über ein Beispiel mit velarem Suffix (mit palatalem gibt es mehrere), in Ts. und in Sav. ist das Abl.-Suffix immer velar, wenn ein velares Wort im Nominativ auf 3 auslautet und dieses velare 3 mit dem anlautenden $i$ des Suffixes zu $\underset{i}{i}$ verschmolzen ist. Diese Tatsache zeugt jedoch nicht gegen die Vokalharmonie, sie weist nur darauf hin, dass zur Zeit der Jahrhundertwende aus der Postposition eus usw. in der DN.-Mundart schon ein Suffix g e w o r d e n war, in den übrigen Mundarten aber die Agglutination eben i m

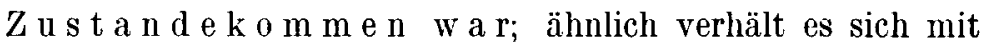
dem Ablativ der Personalpronomina (s. Verf., OstjPron. 251-3), vgl. hierzu auch einige ungarische Dialektformen, wo das Suffix (<selbständiges Wort) mit dem Grundwort nicht harmonisiert: sajáttyáve 'sajátjával; mit seinem eigenen' (Nyr I, 372); kertná' 'kertnél; bei dem Garten', kanálle 'kanállal; mit dem Löffel', pékho 'pékhez; zum Bäcker' (Nyr VII, 417); akárkiná 'akárkinél; bei wem immer auch', táncbe 'táncban; im 'Tanz', sajátjáve 'sajátjával; mit seinem eigenem' (Nyr XI, 
479); istenhoz 'istenhez; zu Gott', keznál 'kéznil; bei der Hand', szójje 'szóval; mit dem Wort', ómánek 'almának; dem Apfel', híromszer 'háromszor; dreimal' (Nyr XIII, 307) usw.

KarJalaines hat den Unterschied zwischen den velar und palatal auslautenden reduzierten 3 -Lauten nicht in Betracht trezogen (s. OL X, KT XXVI), deshalb kann der Lok. mit dem Suffix -nз bei der Untersuchung der Vokalharmonie nicht zur Lösung des Problems beitragen, und deshalb fehlt dieser Fall in Tabelle I.

In Tabelle II und III sind die palatal - velaren Suffixpaare der Possessivsuffigierung bzw. der Konjugation zu sehen, in Tabelle IV einige der in den Texten belegten Ableitungssuffixe.

Die Gesetze der Vokalharmonie im Südostjakischen weichen jedoch von denen des Finnischen und des Ungarischen in gewisser Hinsicht ab. Es wurde bisher vermieden, suffigierte Formen von solchen velaren Wörtern zu zitieren, in denen es ein inlautendes $i, n, t$ oder irgend einen anderen palatalisierten Konsonanten gibt. Wenn man die Deklination der Wörter DN.

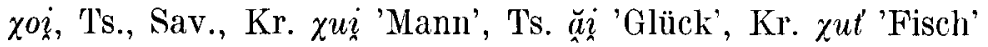
bzw. die Konjugation des Zeitwortes tặida 'haben' überprüft und Ableitungen von velaren Wörtern mit in- oder auslautendem $i, t^{\prime}, n$ usw. zusammensucht, ja sogar suffigierte Formen von vokalisch auslautenden Wörtern (z. B. oxts 'Oberfläche', tă $\gamma_{3}$ 'Ort', tunts 'Birkenrinde'), bei denen vor den vokalisch anlautenden Suffixen ein hiatusfüllender $i$-Laut erscheint, dann findet man überall nach $i, t, \dot{n}$ usw. von diesen Lauten ab bis zum Wortende in der Ts., Sav., Kr.-Mundart n u r palatale Vokale (bis auf die Wörter mit inlautendem $\chi$ nach den erwähnten Lauten), in der DN.-Mundart hinwieder entweder palatale oder velare, s. Tabelle V. Die DN.-Yundart ist also hinsichtlich dieser Erscheinung eine Übergangsmundart zwischen den Ost- und Südmundarten. In den Ostmundarten wird - wie wohlbekannt - die Velarität im allgemeinen durch keinen inlautenden Konsonanten gestört, s. jedoch V. or tä

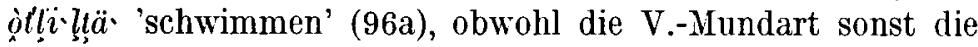
Regeln der Vokalharmonie fast wie das Finnische oder das Ungarische streng befolgt. Auch in den Tavda-wogulischen II undarten (mit strenger Vokalharmonie!) erscheint $e$ statt $\stackrel{a}{,}$ 
bzw. $i$ statt $\varepsilon(e)$ in der nichtersten silbe velarer Wörter nach einem inlautenden mouillierten Konsonanten oder einem $i ;$ wenn es aber auch eine weitere Silbe gibt, so ist diese schon wieder velar (Kannisto, FUF XIV, 55-6, vgl. Steinitz, GWogVok. 54-5).

Die 3. Pers. Sing. des Präteritums wurde bisher auch nicht erwähnt, da sie nur ein einziges Suffix hat: -ot (ohne palatales Gegenstück): DN., Ts., Sav., Kr. iăănot 'er ging', omsot 'er sass', unot 'er lebte', aber auch manot 'er ging', iauot 'er kam', tăijot 'er hatte' (vgl. PAasonen, JSFOu XXI/5, 14 mit 0 : manòt usw., wo o ein "palataaliseerattu takavokaali" ist, a. a. o.). Es sei hier daran erinnert, dass einzelne Beugungsformen ohne Vokalharmonie auch in den Ostmundarten zu finden sind, z. B. das Passiv mit - ui -, ohne palatales Gegenstück.

2. Wir können uns auch mit statistischen Untersuchungen der Frage der Vokalharmonie nähern. Ich habe aus allen Mundarten aus den Texten 10.000 Laute $^{1}$, d. h. 2098 Wörter aus der DN.-Mundart, bzw. 2088 aus der Ts.-, 2210 aus der Sav.- und 2073 aus der Kr.-Mundart statistisch registriert, also ïberall $1 / 4,1 / 3$ oder noch mehr von den uns zur Verfügung stehenden Texten (obwohl gemäss der Wahrscheinlichkeitsrechnung schon $10 \%$ einer genügend grossen Menge die statistisch erfassbaren Eigentümlichkeiten gut widerspiegeln). Die Ergebnisse meiner statistischen Untersuchungen wurden in Tabelle VI zusammengefasst. Als Resultat ergab sich, dass fast $60 \%$ der Wörter in den Texten ganz palatal sind, $30 \%$ ganz velar und ungefähr $10 \%$ der Wörter palatale und velare, Vokale enthalten. Aus Tabelle VI ist auch die Verteilung der Wörter nach ihrer Silbenzahl ersichtlich: die Zahl der Viersilber ist schon ziemlich klein, unter den Fünf- und Sechssilbern, die natürlich noch seltener sind, gibt es überhaupt keine velaren Wörter, nur palatale oder Wörter mit "gemischtem" Vokalismus. Die längsten rein velaren Wörter sind also Viersilber; sollten diese noch weitere Ableitungs-, Possessiv- oder Kasussuffixe bekommen, dann sind diese schon palatal (d.h. das

1 Ich habe das Material nach Laut- und nicht nach Worteinheiten abgegrenzt, weil ich dasselbe Material auch auf die Lautverbindungen usw. untersucht habe; darüher anderswo. 
letzte oder die letzten Suffixe). Hieraus folgt, dass die Zahl der velaren Wörter mit wachsender Silbenzahl rascher abnimmt als die der palatalen. Im allgemeinen ist das Verhältnis palatal/ velar $\approx 2$, bei den Dreisilbern ist pal./vel. $>2$, bei den Viersilbern $>>2$, vgl. Tabelle VII.

In Tabelle VI wurden die Komposita als zwei (Ts. iontdt 'zehn Klafter', Sav. teumo 3 'Schwesterssohn') bzw. als drei (DN. ¿ұa ständig nicht belegt ist, nur in anderen ähnlichen Komposita) Wörter betrachtet, in allen übrigen Fällen wurde die Palatalität, Velarität bzw. Gemischtheit des Vokalismus ganz. mechanisch behandelt. Nach dem eben skizzierten Bild über die Vokalharmonie können aber weder die 3. Pers. Prät. der palatalen Zeitwörter (manot, iza uot 'er ging'), noch Beugungsformen velarer Wörter mit inlautendem $i-, t^{\prime}-, n$-Laut und von diesem an mit palataler Fortsetzung (DN. mońtá 'erzählen',

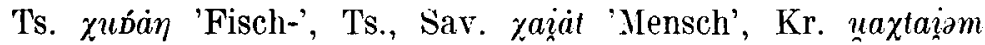
'ich werde gerufen') zu den gegen das Vorhandensein der Vokalharmonie sprechenden Wörtern gerechnet werden. Ja, ein Wort wie z. B. Ts. ăjiasòt 'er bemerkte' entspricht sogar völligr den lokalen Regeln der Vokalharmonie: nach dem $i$-Laut muss das velar beginnende Wort palatal fortgesetzt werden, darum ist ein $a$ und nicht ein $a$ zu sehen, und die Endung der 3. Pers. Sing. ist im Prät. immer -ot. Fremdwörter, wie nådo, iaș̌o,

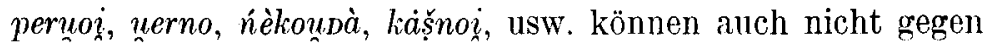
die Existenz der Vokalharmonie zeugen.

Es gibt in dem Textnachlass einige Wörter scheinbar ohne Vokalharmonie: DN., Sav. iauai 'er kam', aber mit Vokalharmonie zehnmal DN., viermal Sav. zaua ä; DN. әnдam 'nein', aber $17 \mathrm{mal}$ andám; 'Ts., Sav. iăran 'Samojede', aber im 'Ts.Text wurde auf derselben Seite, wo dieses Wort mit "ggemischtem" Vokalismus zul finden ist, 4mal und auch in der

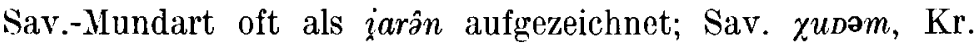
$\chi \bar{u} t ə m$ 'drei', s. jedoch $11 \mathrm{mal} \chi u D \partial \hat{m}, 26 \mathrm{mal} \chi \bar{u} t \hat{\partial} m$ usw. M. E. müssen nicht nur diese Wörter ohne Vokalharmonie unbedingt als Schreibfehler betrachtet werden, sondern auch solche wie die erste Angabe mit gemischtem Vokalismus in den folgenden Beispielen: Ts. nặməsat : năı̆âsat 'mit dem Gedanken', Kr. 
lăxren: tă̌xreu 'unser Panzerhemd', obwohl die meiner Ansicht nach korrekte Form nur einmal in den Texten vorkommt.

Wenn man die palatalen Zeitwörter mit der Endung -ot, die velar beginnenden Wörter mit inlantendem $i, t$ usw. und die sicher wegen eines Schreibfehlers nicht den lokalen Gesetzen der Vokalharmonie entsprechenden Wörter nicht zu den Wörtern zählt, die der Existenz der Vokalharmonie widersprechen, so verringert sich die Zahl der vokalharmonielosen Wörter (d. h. mit "gemischtem" Vokalismus) bedeutend: es bleiben nur DN. $3,4 \%$, Ts. $0,96 \%$, Sav. $1,6 \%$, Kr. $1,5 \%$ Wörter, die sogar auch nach den eben festgestellten "Regeln" der Vokalharmonie in den südostjakischen Mundarten als Wörter ohne Vokalharmonie zu betrachten sind. Bei allen diesen Wörtern könnte man mit Hilfe eines diakritischen Zeichens (Setzung oder Streichung) die Vokalharmonie vin Ordnung» bringen, aber eine solche Korrektur würde ich dennoch nur dann vorschlagen, wenn ein palatales Wort, vermutlich infolge eines Schreibfehlers, ein velares Suffix hat, in den übrigen Fällen nicht. Die anderen Wörter ohne Vokalharmonie weisen nämlich eine gewisse Regelmässigkeit auf: sie haben in der ersten oder in den ersten Silben velare Vokale, dann kommt ein $i$-Laut ${ }^{1}$, oft stehen nach velaren Wörtern palatale Possessivsuffixe, oder Possessiv- und Kasussuffixe, bei den Zeitwörtern sind oft nur die Personalsuffixe palatal oder das Zeichen der Optativform, oft die Partizipialendung, der noch Possessivund Kasussuffixe folgen; das Gerundium lautet immer auf -men aus, vielleicht beginnt in der Sav.-Mundart der Dual auch nur eine palatale Endung $(-\gamma \partial n)^{2}$ zu haben; die Post-

1 In wogulischen Mundarten mit Vokalharmonie kommt auch ein $i$-Laut bei velaren Wörtern in Suffixen vor, s. I.U., LM., VN., VS., VNK. (KANnisto, FUF XIV, 59, 63, 66-7, 68, 69).

2 Eine ähnliche Erscheinung gibt es auch im Wogulischen; in der LM.-Mundart treten die Verbalsuffixe -kăt, -taxt an velare und an palatale Verbalstämme, obwohl sonst in dieser Mundart die Vokalharmonie gut zu beobachten ist (auch die erwähnten Verbalsuffixe weisen ein palatales Gegenstück in der LU.-Mundart auf, vgl. KaNNisto, FUF XIV, 64). Einige Suffixe hatten um die Jahrhundertwende in der VS.- und VNK.-Mundart schon ihre velare Entsprechung verdrängt (KANNISTo, op. cit. 68-9, 70-1); einige andere hinwieder 
positionsartigkeit des Ablativsuffixes in der 'Ts.-, Sav.- und Kr.-Mundart wurde schon erwähnt. Ausserdem sind die Wörter ohne Vokalharmonie zumeist längere Wörter. Ebendeshalb dürfte es wohl nicht nur dem reinen Zufall zuzuschreiben sein, dass Suffixe velarer Wörter in der vierten Silbe of $\mathrm{t}$, in der fünften Silbe i $m$ m e $r$ palatal sind. Dass dem so ist, und dass diese letzte oder letzten palatalen silben nicht immer Schreibfehler sind, ist daraus ersichtlich, dass Kar.IALAINEN ab und zu diese palatalen Vokale der letzten Silben in velar beginnenden Wörtern zweimal unterstrichen hat (z. B. Sav. uăttâpá, '[in seine Feldhütte]', S. 19, zwischen den Zeilen

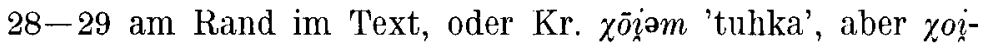
$m \hat{\eta} \eta$ adj. in der Aufzeichnung der Wörtersammlung, Spalte IV 11b).

Es ist möglich, dass vielleicht noch irgendwelche Zusammenhänge zwischen der phonetischen Umgebung und dem "gemischten" Vokalismus festgestellt werden könnten, die ich bisher nicht bemerkt habe. Es gibt ja auch im Ungarischen schwer einreihbare Fälle der Harmonie, z. B. ist der Vokalismus in den ungarischen Wörtern kerék 'Rad' und derék 'Rumpf' ganz derselbe, auch die phonetische Umgebung ist ziemlich die gleiche; bei der Suffigierung werden an das erste Wort jedoch ausschliesslich palatale, an das zweite Substantiv hingegen nur velare Suffixe gehängt (kerekek, kereket, kerekem, kerekes bzw. derekak, derekat, derekam, derekas usw.). Das ungarische Wort fiu 'Knabe' ist ein velares Wort, mit dem alle Suffixe nur in ihrer velaren Form verbunden werden: fiaim, fiak, fiúkat usw. Das verblasste oder verblassende Kompositum férfi( $u)$ 'Mann' $<$ férj 'Gatte' + $f i(u)$ ist in der Form férfiú auch ein velares Wort (férfiúnak, férfiúhoz), aber in der kürzeren Form férfi beginnt es zur Palatalität überzugehen. Der Plural wird noch immer mit velaren Suffixen versehen: férfiak, férfiaknak (die Formen férfik, férfiknek existieren zwar, sind aber noch keine

erscheinen in VNK. nur hintervokalisch (ebd. 71). Auch in den ungarischen Dialekten haben einige Suffixe ab und zu nur eine Form, die dann mit dem Grundwort nicht harmonisiert, z.B. kicsika 'kicsike; kleinwinzig', nénika 'nénike; Tantchen' (Nyr XIII, 307). 
schriftsprachlichen Formen), neben férfias 'männlich', férfia 'sein Mann', gibt es sogar keine Formen mit palatalen Suffixen; aber s. férfinak $\sim$ férfinek, férfiban férfiben usw., im Singular gibt es also neben den velaren auch schon rein palatale Formen (vgl. A magyar nyelv történeti-etimológiai szótára). Vielleicht verhält es sich ähnlich im südostjakischen, wo einige velare Wörter mit velaren und palatalen Suffixen belegt sind und wo bisher keine "Regelmässigkeit» entdeckt worden ist ${ }^{1}$.

3. Es gibt noch einen weiteren Grund, weshalb ich diese wenigen Wörter ohne Vokalharmonie $(0,96 \%-3,4 \%) \mathrm{n} \mathrm{i} \mathrm{c} \mathrm{h} \mathrm{t}$ a $11 \mathrm{e}$ korrigieren möchte: in KaRJALaINENs Wörterbuch gibt es bei den velaren Zeitwörtern oft fakultativ angegebene velare und palatale Endungen (s. Faludi, op. cit. 608, 609). Bisher habe ich a us chliesslich die 'Texte in Betracht gezogen; um aber zu einem möglichst endgültigen Ergebnis in der Frage der Vokalharmonie zu gelangen, muss man natürlich nicht nur die Texte, sondern unbedingt auch KarJalainens Wörterbuch gründlich untersuchen. Dazu soll aber erst genau der Vokalwert der einzelnen Vokalzeichen im Wörterbuch und das Verhältnis der Zeichen in der Originaltranskription der Texte zu dem in der Ausgabe des Wörterbuches festgestellt werden.

Wenn ich behaupte, dass die Texte eine strenge Vokalharmonie aufweisen, habe ich alle Vokalzeichen als Merkmale von palatalen bzw. von velaren Vokalen aufgefasst, je nachdem, wie ich es mir aus Karjalarnens Definitionen der Vokalzeichen (OL S. VIII-X, bzw. KT S. XXV-XXVI) auslegen konnte. Bei $a, o, u$, bzw. $e, i$ und bei den reduzierten

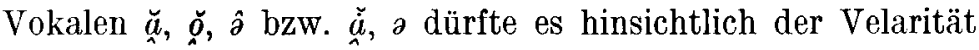
bzw. Palatalität wohl keine Probleme geben. Alle Vokale, unter denen, steht, oder über denen ' ist, habe ich als palatalc Vokale betrachtet, ich habe also $\dot{a}, o, u$ und die in den Texten nur vor, nach oder zwischen palatalisierten Konsonanten ver-

1 Es gibt auch im Wogulischen scheinbar irreguläre harmonielose Wortformen in Mundarten mit Vokalharmonie; hier begründet KANNisto sprachgeschichtlich ihr Zustandekommen (TJ., TC., LU. s. FUF XIV, 54, 60). 
wendeten Vokalzeichen $\dot{o}, \dot{u}$ zu den Merkmalen der palatalen Vokale gerechnet ${ }^{1}$, diejenigen hingegen, unter denen , oder

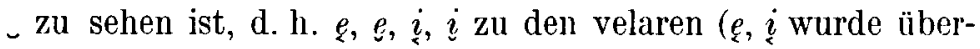
wiegend in den Aufzeichnungen an der Demjanka und an der Konda, $e$, ? hingegen am Irtysch benutzt).

Es ist wohlbekannt, dass K.ARJALAINEN seine Transkription bei der Bearbeitung seiner umfangreichen Sammlung in mancher Hinsicht verändert hat. Auch Torvonen hat KarJaLainens grosses Wörterbuch nicht in der Originalaufzeichnungsweise, sondern mit derselben Transkription publiziert, die KaRJalaINEN in seinen Publikationen (OL, Wie e g 0, usw.) benutzt hat. Ebendeshalb habe ich alle Wörter der Texte (die natürlich im grossen und ganzen in derselben Transkription aufgezeichnet wurden wie die Wörtersammlungen) mit den betreffenden Wörtern des Wörterbuches verglichen, um die Transkription der V o k a le zur Publikation der Texte gemäss der des Wörterbuches zu verändern. ${ }^{2}$ Dabei erwiesen sich viele Vokalzeichen ( $a, \dot{a}, o, u, e, i, \dot{u}, \partial, \hat{\partial})$ als ganz problemfrei; bei den reduzierten Vokalen $\underset{a}{\breve{a}}, \breve{a}, \stackrel{o}{a}$ fehlt in den Texten ziemlich oft (aber wie aus dem Vergleich der verschiedenen Aufzeichnungen der sich wiederholenden Wörter ersichtlich, nicht immer) das Zeichen der Reduziertheit. Den grössten Schwierigkeiten bin ich in der Kr.-Mundart begegnet, wo [ü] und [ô̆], genauer gesagt ihre Bezeichnungsweisen ganz durcheinandergeraten sind, was aus Abbildung 1 zu ersehen ist.

Wie aus Abbildung 1 ersichtlich, gibt es hier Vokalzeichen, die in den Originalaufzeichnungen und im wörterbuch vorkommen $\left(\dot{u}, u, u^{3}\right)$, weiterhin solche, die nur in den Originalaufzeichnungen $(\underset{b}{\sim}, \dot{u})$ oder nur im Wörterbuch $(u, \underset{o}{\breve{o}}, \dot{g})$ verwendet worden ${ }^{\delta}$ sind. In einer Hinsicht dürfte jedoch kein Zweifel bestehen: alle Zeichen der Abbildung kommen nur in palatalen Wörtern vor ( bis auf das selte n e Zeichen $u$, wo die übrigen Silben in

1 In den Texten kommt $\underset{0}{\breve{O}}$, $\check{O}$ usw. nicht vor, s. unten S. 26--7.

2 In der Originalaufzeichnung wären heute nicht alle Vokalzeichen setzbar, vgl. MSFOu 128: $\mathrm{X}$, ff.

${ }^{3} u$ kommt im KT zwar vor, aber nur in der Ts., Koš., und T'š.Mundart, in Kr. nicht. 


\section{Abbildung 1}

Kr.--Iundart

KT. [ii]

Mser.

KT. [ọ̈]

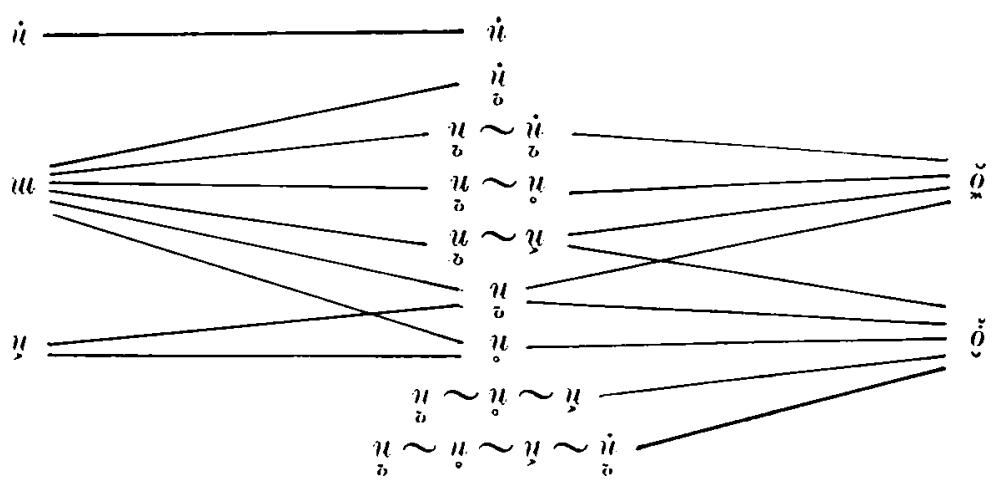

den Texten aus Krasnojarsk abwechselnd als palatal oder velar aufgezeichnet wurden; die entsprechenden Wörter im Wörterbuch sind aber in der Kr.-Mundart und sogar auch in den übrigen Mundarten immer palatal.

Meine Auffassung hinsichtlich der Palatalität oder Velarität der einzelnen Vokalzeichen steht also in vollem Einklang mit der Karjalanness, der die Vokale in OL in der folgenden Reihenfolge behandelt hat: $\dot{a}(1,243), \stackrel{a}{a}(24,245), a(28,245)$, $\breve{a}(44,247) ; o(70,249), \stackrel{o}{o}(78,250), o(87,250), \stackrel{o}{o}(109,254)$; y $(124,255), u(136,256) ; e(155,257), e(177,260)$, , 。 $261 ; 205,262) ; i(208,263)$; d. h. bei einer jeden Lautgruppe (bis auf die $e$-Laute) beginnt er mit dem palatalen vollen Laut, dem folgt der palatale reduzierte (wenn es einen solchen gibt), dann kommen die entsprechenden velaren vollen und reduzierten Laute (bei den $e$-Lauten ist $e, e, a\left({ }_{o}\right)$ die Reihenfolge, d. h. voll - palatal, velar —; reduziert); $\hat{\partial}$ kommt in der ersten Silbe nicht vor, dem entspricht $\breve{a}$, das wiederum fast nur in der ersten Silbe belegt ist. Schematisch dargestellt können, um KARJALAINExs Verfahren zu veranschaulichen, die Laute untereinander in Kolumnen geordnet werden (s. Abbildung 2.). 


\section{Abbildung 2.}

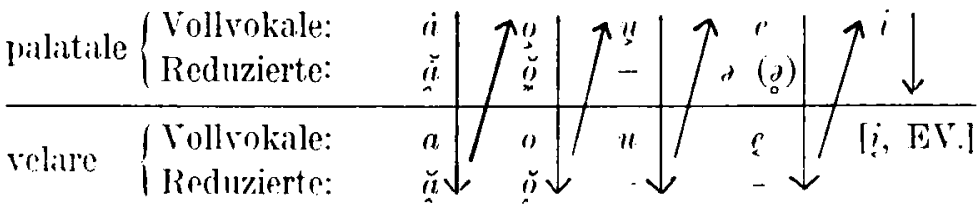

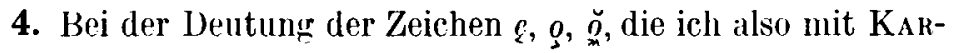
JALAINEN für velares $e$ bzw. für palatale volle und reduzierte $\ddot{o}$-Laute $([\ddot{o}],[\ddot{o}])$ halte, widerspreche ich Steisitz, der diese für Varianten von $[e],[o],[\check{]}]$ hält. Dieses Problem muss nun unbedingt gründlich überprüft werden; es handelt sich einerseits darum, ob es in den südostjakischen Dialekten so viele Laute gibt, wie KarJalaines festgestellt hat, oder um drei $(\varrho, o, \underset{\infty}{)})$ weniger, wie Stresnitz meint; andrerseits muss die Frage beantwortet werden, ob in einem System, wo Vokalharmonie herrscht, velare Laute palatale und palatale Laute velare Varianten haben können oder nicht.

4.1. Fangen wir mit dem velaren $e$ an. Ich zitiere die Meinung von Steinitz: w o r $\chi$, in welcher Stellung $e$ etwas weiter hinten artikuliert wird, schreibt er [d. h. KARJALAINEN] ę" (Geschichte des ostjakischen Vokalismus, im weiteren GOstjVok., 23). Wenn man aber das ganze einschlägige Material aus KT zusammenstellt, muss man diese Behauptung schon etwas ergänzen, $e$ kommt nämlich in der ersten Silbe ganz sicher vor $-\eta$, vielleicht sogar vor $-r$ vor (s. Tab. VIII ${ }^{1}$ ); s. noch DT. ę:

Als Steinitz seine Geschichte des ostjakischen Vokalismus verfasste, ja als er die Korrekturen las, konnte er aus KARJALAINENS reichem Material nur OL gut kennen, das Wörterbuch konnte er, wie aus seiner Korrekturnote (Vorwort, Anm. 2) ersichtlich, nicht einmal beim Korrekturlesen in die Hand bekommen. Wie wohlbekannt, sind in OL die Nomina immer im Nom. angegeben, von den Zeitwörtern nur der Stamm

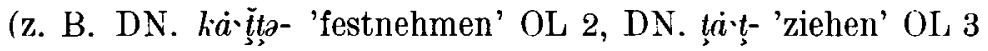

1 Die Belegstellen zu den 'l'abellen VIII-XII, XV, XVIII-XXIII s. bei den Tabellen, S. 46-70. 
usw.). Es muss noch nachdrücklich darauf hingewiesen werden, dass KarJalainen und Steinitz in ihren zitierten Werken nur den Vokalismus der ersten Silbe behandeln und sich demgemäss für suffigierte Formen nicht interessierten. Wenn man aber aus dem Wörterbuch die phonetische Umgebung der e-Zeichen in der 2. Silbe (Tab. IX-XI), ja sogar der 3. Silbe (Tab. XII) zusammensucht, kommt man zu dem Resultat, dass $e$ vor und $\mathrm{nach}$ allen Konsonanten vorkommt. (In den Tabellen weist ein Kreuz auf $\underline{\text {, ein Kreis }}$ auf $e$ hin, und schliesslich ein Kreis mit eingeschriebenem Kreuz auf $e$ und auch auf $e$ ). Die Tabellen enthalten natürlich nur die konsonantische Umgebung, es muss aber auch noch, sogar mit grossem Nachdruck, darauf hingewiesen werden, dass a lle Wörter, die in der 2. oder 3 . Silbe einen $e(e)-L a u t$ a u f w e isen, in der 1 . bzw. in den beiden ersten Silben auch nur velare L a u te haben (s. im Anhang die Beispiele zu den Tabellen $(\mathrm{X}-\mathrm{XII})^{1}$, in de $\mathrm{n}$ Wörtern hingegen, wo $e$ (e) in der ersten Silbe $\mathrm{zu}$ finden ist, gibt es mindestens in der zweiten, oder auch in den weiteren Silben, wenn solche vorhanden sind, nur velare L a u t e (s. OL 177-8 $\hat{\partial}, e, a$ in der zweiten Silbe bzw. das "neutrale" -3 im Auslaut der zweiten Silbe, weiterhin s. im Anhang unter den Beispielen zu der Tabelle VIII die Mehrsilber bzw. die suffigierten Wortformen).

STEINITz meint, dass "die Qualität der nichtersten Vokale im Fiugr. bei der herrschenden Vokalharmonie ${ }^{1}$ [1...] wesentlich durch die Qualität des ersten Vokals bestimmt» wurde (GFgrVok. 3). Das Ostostjakische - m. E. auch das Südostjakische - ist »eine Sprache mit streng durchgeführter Vokalharmonie ..., in der der Vokalismus der nichtersten Silben vom Vokalismus der ersten Silbe bestimmt» wird (GOstjVok. 11). Ausserdem muss eine andere Behauptung von STEINitz auch

1 Im Wörterbuch werden mehrere Wörter so angegeben, als könnten sie in der nichtersten Silbe ein $e_{(q)}$ und weitere velare Silben haben, o d e r $e+$ weitere palatale Silben; verschiedene, hauptsächlich zweisilbige Suffixe treten oft auch nach $\underset{\varsigma}{e}(e)$ in ihrer palatalen Form an die Wörter. 
unbedingt anerkannt werden. "In den fiugr. Sprachen kommen - oder kamen ... nur in der ersten Silbe alle in der gegebenen Sprache vorhandenen Vokale vor) (GFgrVok. 3), und genau so im Ostjakischen: "in der ersten Silbe kommen alle Vokalphoneme vor, in den nichtersten Silben nur ein Teil" (GOstj. Vok. 11). Da nun im Südostjakischen $e$ in der nichtersten Silbe nur in Wörtern mit velarer erster Silbe vorkommt, und wo $e$ in der ersten Silbe steht, die weiteren Silben auch ve l a r si n d, ist $e$ - wie aus den übrigen Silben ersichtlich - das Zeichen eines velaren Lautes. Weiterhin ist $e$ in der nichtersten Silbe sicher nicht positionsbedingt, es kann vor und nach verschiedenen Konsonanten erscheinen: so ist es sicher keine Variante (sogar eines palatalen Lautes), sondern ein selbständiges, velares Phonem.

Nach den hier zusammengefassten Ergebnissen scheint es mir logisch, die Personal- und Possessivsuffixe -ęm, -ęn usw. bei velaren Wörtern, bzw. -em, -en usw., $\mathrm{n}$ i c h t als fakultative Varianten, sondern als velare - palatale Suffixpaare zu brtrachten, die an velare bzw. palatale Wörter gehängt werden.

Um ganz deutlich, unanfechtbar zu beweisen, dass $e$ bzw. e (e) zwei voneinander verschiedene Phoneme sind, müssten zwei verschiedene Wörter angeführt werden, in denen die Phoneme $e: e$ "Träger des Unterschiedes" wären (wie in den ungarischen Wörtern bab 'Bohne': báb 'Puppe' die Phoneme $a$ : $\dot{a}$, oder im Finnischen bei kuu 'Mond, Monat': kyy 'Kreuzotter' $\bar{u}: \bar{u})$. Die Zahl der Einsilber mit $\ell$ ist aber im Südostjakischen sehr beschränkt, wir kennen nur die in Tabelle XIII aufgeführten. In allen Beispielen in Tabelle XIII deutet die Schreibweise der dem Vokal folgenden Konsonanten $(-\chi,-\eta \eta \chi)$ auch unmissverständlich darauf hin, dass die Wörter velar sind. Ein genaues palatales Gegenstück in derselben Mundart kann man ganz sicher nur bei dem zweiten der zitierten Wörter finden, die Bedeutung der palatalen und der velaren Wörter ist aber genau dieselbe, sie beweisen also hinsichtlich der Zeichen $\ell$ : $e$ nichts, sind nur velare - palatale expressive Wortpaare. Bei DN. rę\% (263b), welches unter eigenem Stichwort als DN. rè $\chi(795 \mathrm{~b})$ erscheint, kann man etwa an einen Druckfehler denken. 


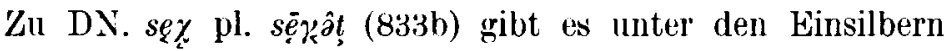
kein palatales "Gegenstück", aber unter den Mehrsilbern gibt

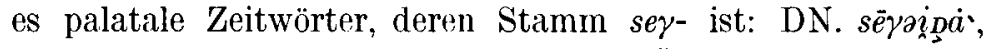

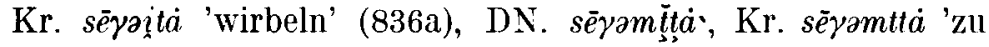

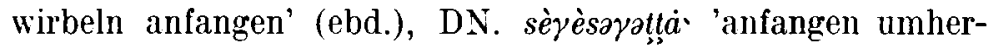

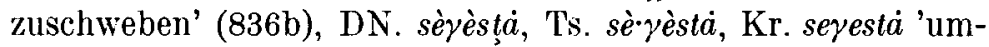
herschweben' (ebd.).

Zu den übrigen Beispielen gibt es keine pallatalen Gegenstücke. Bei der nur oberflächlich bekannten DT.- und Koš.Mundart ist dies fast selbstverständlich. Zu unserem letzten Beispiel könnten nur solche mit elk-beginnenden Wörter als Gegenstücke gestellt werden, wo e $k^{k-}$ nur ein Teil des Wortstammes ist: DN. e'narọa', Kr. enkarta '(ver)fluchen' (37b) usw., die aber genau so gut (oder genau so schlecht) dem

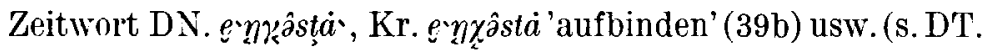
e'nkita 'losmachen' 39a) gegenüber beweisen können, dass man palatale - velare Wortpaare finden kann, deren erste Silben nur in der Palatalität - Velarität voneinander abweichen.

Die Zwei-, bzw. Dreisilber können, wie aus dem oben (s. S. 12) Gesagten ersichtlich, nicht geeignet sein, zu entscheiden, ob ein Vokal ein Phonem ist oder nicht; die Wörter, in denen ein $e$ in der ersten Silbe steht, weisen in den übrigen Silben nur palatale Vokale auf, wo sich aber in der ersten, zweiten oder dritten Silbe ein $\ell(e)$ befindet, gibt es in den übrigen nur velare Selbstlaute. So gäbe es hier überall, falls die Konsonanten dieselben wären, ausser dem Unterschied $e$ : $\ell$ mindestens noch eine Abweichung im anderen Vokal (oder in den anderen Vokalen). Zu den auf fast "neutrales" -3 auslautenden Zweisilbern - DN. $i \bar{e} \gamma_{\alpha}^{3}$ 'Fluss' (OL 177, 146b), Kam. $t^{\prime} \chi_{x 3}$ '... Wurst' (899b) sind keine palatalen "Gegenwörter» bekamnt.

Schliesslich bleibt noch ein Wort, wo in der ersten und auch

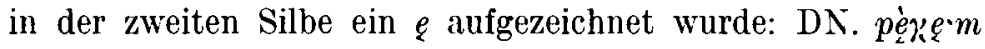
'verfault' (OL 177), dieses hat aber vielleiclit eine palatale

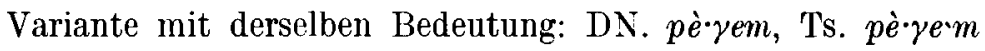
'sauer geworden', s. noch DN. pèyem (668a); es ist aber auffallend, dass in dem von Torvones publizierten Wörterbuch eben die in OL belegte Variante nicht $\mathrm{zu}$ finden ist! Wir haben hier wahrscheinlich ein ursprünglich velares Wort vor 
uns (V. pilii $m$;, vgl. Vj., Trj.), welches in einigen der Südmundarten palatal geworden ist, oder noch eine palatale und eine velare Variante hat.

Mit der konventionellen Methode, d. h. mit einem Wortpaar, dessen "Unterschiedsträger» die Phoneme $e$ : $e$ wären, kann also nicht bewiesen werden, dass das in der ersten Silbe schwach belastete Vokalzeichen $e$ ein Phonem ist. Da wir aber aus der DN.-Mundart nur 5-6, aus der Kr.-Mundart 3, aus der DT.-, Koš.-, Kam.--Ilundart je ein Wort kennen, wo als Vokal nur $e$ (bzw. - - - und -3) zu finden ist (und es unter diesen auch expressive Wörter gibt, wo palatale - velare Wortpaare möglich sind), so wäre es wirklich ein Zufall, wenn dieselben Konsonanten mit $e$ in einem anderen Worte auch vorkämen. Dass aber $\&$ keine Variante von $e$ sein kann, ergibt sich daraus, dass $e$ nur in velaren, $e$ hinwieder (besonders in den ersten Silben) nur in palatalen Wörtern erscheint.

Deskriptiv hat die Tatsache keine Beweiskraft, aber in historischer Hinsicht ist es dennoch nicht belanglos, darauf hinzuweisen, dass den "velaren" südostjakischen Wörtern mit einem $\varrho(e)$ im Wortstamm, in den Ostmundarten auch immer nur velare Wörter entsprechen (OL 177-8) und dass die südostjakischen Wörter mit palatalem $e$ hingegen zumeist (bis auf die mit ze- anlautenden Wörter) ursprüngliche palatale Wörter sind (s. die Entsprechungen in den Ostmundarten, OL $155-69,174-6)$.

4.2. Karjalainen und Steinitz erwähnen nicht einmal das Problem des velaren $i$-Lautes. M. E. gibt es, genauer gesagt g a b e s das noch zur Zeit der Jahrhundertwende und früher. In der ersten Silbe ist dieser Laut äusserst selten, in OL kommt er überhaupt nicht vor, aus dem Wörterbuch kann ich auch nur die Beispiele in Tabelle XIV zitieren.

Ableitungen von den Angaben in der Tabelle XIV sind nur

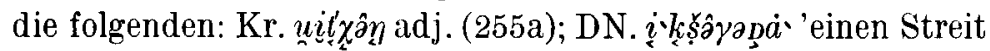
anfangen' (31a); DN. ìrâsțà̀, îroṣ̆ta' 'einen Streit anfangen' (78b).

KarJalainex und Steinitz erschlossen für das Urostjakische ein velares $i$, aus welchem im Südostjakischen zumeist $i$ geworden ist (OL 283, GOstjVok. 78-9, 96). Die ersten drei zitierten Beispiele in Tabelle XIV könnten etwa als 
archaische Relikte betrachtet werden, wo neben $*_{i}>i$ noch $*_{i}>\underline{i} \sim i$ zu finden ist, ein vermutlich entsprechendes Wort aus den Ostmundarten kann nur zum ersten gestellt werden, wo

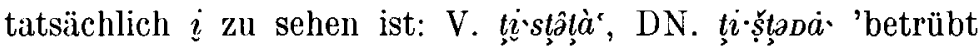
sein' (1028b; 'Ts., Kr. $i \sim$ Trj. i); m. E. dürfte nämlich Kr. tiṣ̆tam, tiṣ̆tam 'schroff ablehnende Erwiderung auf einen Befehl' (1029a) vielleicht hierher gehören.

Bei den zwei Beispielen mit DN. $i \sim i$ widersprechen die Entsprechungen in Kr. bzw. auch in Ts. weder einer Erschliessung von $*_{i}$ noch der von $*_{i}$ (OL 283, GOstjVok. 77,78); der Beleg mit $\ell$ in DT. kann aber nach KarJalaineN (OL 284) nur aus $*_{i}$ hergeleitet werden; nach dem oben Dargelegten (S. 11-5) teile ich in dieser Frage unbedingt seine Meinung. Im vierten der in der Tabelle befindlichen Beispiele ist das Schwanken $i \sim i\left(<^{*} i\right)$ interdialektal bewahrt (vgl. Kam., Sogom mit i). Es sei noch bemerkt, dass dieses Wort etwa wegen seiner Expressivität den velaren $i$-Laut in $\mathrm{Kr}$. bewahrt haben kann, wir kennen nämlich ein anderes Beispiel, wo in der Kr.-Mundart in genall derselben phonetischen Umgebung dem ïstlichen $i$ ein $i$ entspricht: Vj. uit's' 'Fischdärme' $\sim$ Kr. ui.t': u. serkita 'Fischdärme 11.a. zerhacken' (253b, Trj $i$ ebd., V. $i 25+\mathrm{a} ;$ V. $u \dot{u} \cdot t^{\prime} 253 \mathrm{~b}$ s: $u \underline{i} \cdot t^{\prime} \mathrm{s}$. OL 220 ).

In einem unserer Beispiele entspricht dem südostjakischen ? im Osten ein $i$; Kam. rịm $\chi_{z}, \mathrm{~V}$., Vj. rimak, Trj. ri merung' (804a). Da wir alls KarJalainess Aufzeichnungen über die Kam.-Mundart nur sehr spärliche Kenntnisse haben, kann ich mir über dieses Wort nur dann eventuell eine Meinung bilden, nachdem ich PaAsoness Textnachlass durchstudiert habe, vgl. KarJalainex (OL 168).

Ich würde noch ein Wort hier erwähnen: bei Ts. nìr muss man vermutlich auch von einem urostjakischen palatalen Laut ausgehen: ${ }^{*} \dot{u}$ (OL 281) bzw. in der Transkription von Steinitz $*_{\ddot{u}}$ (op.cit. $79-80,96$ ). Im Wörterbuch fehlen hier $(590 \mathrm{~b})$ Angaben aus anderen Südmundarten. Nach den zu derselben Vokalentsprechungsgruppe gehörenden Beispielen bei KARJALAINen (OL 213) wäre in der Ts.-Mundart auch ein $i$ zu erwarten, vgl. KT 465a, 485a, 520a, 675a, 780a, 829a, 973b, 1047a usw. (sogar bei V., Vj., Trj. $\underline{i} \sim \mathrm{DN}$. $i$ ist Ts. $i$ zu finden 
s. ()L 217-8 b'ww. K'l 112a, 489b, 722a, 812a, 948a; 181b, 189b-190a, 629a, 649a, 625a). Ich würde Ts. nịr jedoch zu DN. ni.r 'Latte, Stange' (590b) stellen, da es der Bedeutung nach - i [ŭ̆Dâm] nî̀rât 'nuotan ahdepuut' (197b) - gut hierher passt: es könnte vielleicht angenommen werden, dass die Velarität des zweiten Wortes dem Einfluss des ersten Gliedes des Syntagmas zuzuschreiben ist.

Schliesslich sei noch erwähnt, dass zwei DN.-Lehnwörter (oder Fremdwörter) nicht mit $i$ aufgezeichnet wären, wenn dieser Laut der DN.-Mundart ganz fremd wäre; dann wäre das russische bu durch einen anderen Laut ersetzt.

In der zweiten Silbe kommt $\underset{i}{i}(\underset{)}{)})$ häufiger vor, und zwar vor und nach allerlei verschiedenen Konsonanten (s. Tabelle XV), aber nur in Wörtern mit einem velaren Vokil in der ersten Silbe.

Beispiele mit $i$ in der dritten Silbe gibt es auch, aber nur

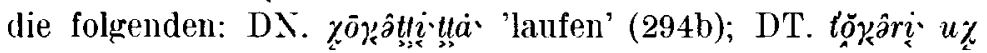
'ein Gerät zum Glätten' (902a); Kr. nūmânįı, -it 'ylempänä asuvat' (567b). Die ersten zwei Silben sind überall unanfechtbar velar. In der vierten Silbe kommt $\underset{i}{i}$ auch lediglich nach vorhergehenden velaren Vokalen vor: DN. ī öffnen' ( $66 \mathrm{~b})$.

Ich glaube, als KarJaLainen unter den Ostjaken sein Material sammelte, könute es noch einen velaren $i$-Laut als $V$ a r i a n t e des $i$-Phonems gegeben haben; es könnte jedoch ein Vokal gewesen sein, der wegen seines äusserst seltenen Vorkommens bereits im Begriff war, gänzlich aus dem Vokalsystem zu verschwinden. Stand er ursprünglich in der ersten Silbe, dann entstanden palatale - velare Wortpaarvarianten (s. DN., Kr.), stand der $i$-Laut in der nichtersten Silbe, dann konnte das betreffende Wort entweder velar oder palatal enden (s. Anhang zu Tabelle XV). Der Lautwandel $i>i$, d. h. das Aussterben, Verschwinden des velaren $i$-Lautes könnte sich Anfang unseres Jahrhunderts vollzogen haben, und als STEINitz um mehr als drei Jahrzehnte später als KakJaLAINex das Südostjakische hörte, dürfte es einen solchen Laul wohl nicht mehr gegeben haben.

Da heutzutage weder das Ungarische noch das Finnische einen velaren $i$-Laut hahen, obwohl in beiden sprachen eine 
strenge Vokalharmonie zu finden ist, und in beiden Sprachen der $i$-Laut in palatalen und velaren Wörtern oft anzutreffen ist, so kann der Lautwandel $*_{i}>i$ im Südostjakischen mit dieser Analogie gestützt werden. Es ist selbstverständlich einerseits, dass in velaren Wörtern nach einem $* i>i(\sim i)$, falls ein $i$ vorkommt, velare oder palatale, falls ein $i$, nur palatale Vokale folgen konnten, andrerseits, dass dadurch die Wörter mit ${ }^{*} i>$ $i$ zum Übergang des Wortendes ins Palatale beitragen konnten.

4.3. Wie wohlbekannt, stellt STEIxitz die Existenz irgendeines vollen oder reduzierten $\ddot{o}$-Lautes im Südostjakischen in Abrede (s. oben S. 11, GOstjVok. 22, 1, 24, 96-7, GFgrVok. $66,68,70-1$ ). Über KarJalainens o (genauer gesagt DN., Ts. $\stackrel{o}{,}, \mathrm{Kr} . \dot{g}$; neben palatalisierten Konsonanten, hauptsächlich in Kr., $\dot{0}$ ) ist er der folgenden Meinung: "Es handelt sich bei Karj. DN o klar um eine kombinatorische Variante, ein etwas palatalisiertes $o$, das neben dem allgemein palatalisierenden $k$ und vor mouillierten Konsonanten sowie dem dem $k$ entsprechenden Nasal $\eta$ vorkommt. - Dasselbe gilt von Paas. Kond. $\overline{\dot{o}}^{1}$, das ebenfalls neben $k$, vor $\eta$ und mouillierten Konsonanten erscheint, ausserdem noch vor $i$, in welchen Stellungen Paas. ó nicht vorkommt» (GOst.jVok. 24, GFgrVok. 68, Anm. 11). Dasselbe behauptet er anderswo auch über KarJalainens "Varianten", wo er meint: KarJalaines unterscheide "in DN ein hinteres $o\left(<^{*} a\right)$ und ein vorderes $g$; das letztere kommt aber nur neben $k$ und vor mouillierten Konsonanten und $\eta$ vor, in welchen Stellungen wiederum $o$ nicht erscheint; Karj. DN $g$ ist also eine klare kombinatorische Variante" (GOstjVok.1), ausserdem: "Bisweilen schreibt Karjalainen jedoch DN $o$ (70 f.), und zwar nur neben $k \ldots$ und vor $\eta \ldots$ oder mouillierten Konsonanten $(n, t: 8 \times)$, in welchen Stellungen Karj. o nicht rorkommt» (op. cit. $23-4$ ).

Die Behauptung von Steivirz, dass o nicht vor denselben Konsonanten vorkomme wie $o$, kann schon aus dem Material der OL' selbst widerlegt werden ${ }^{2}$ (s. die Tabellen XVI, XVII).

1 PaAsonens o bezeichnet einen o-Laut, kein [ö] (vgl. oben S. 4, JSFOu XXI/5,14, PD XIV).

2 Neben $k$ kommt $o$ tatsächlich nicht vor. Näheres hierüber s. unten, S. 34 . 
Um aber zu beweisen, dass ein [ö] bei KaRJALAINen auch in anderen Umgebungen erscheint, als STEINiTz schreibt, soll auch das Wortmaterial des Wörterbuches herangezogen werden (s. Tabellen XVIII-XX).

Tabelle XVI gibt eine Übersicht über die interdialektalen Entsprechungen im Süden der Lautgruppen DN. -oi, -on und -ot', die Steiniz'z aus OL und aus Paasonens Wörterbuch schon kennen konnte, als er seine Geschichte des ostjakischen Vokalismus verfasste. Aus KarJaLainess Wörterbuch wurden $\mathrm{n} \mathrm{u} \mathrm{r}$ die in OL oder in PD befindlichen Angaben aus weiteren Mundarten ergä $\mathrm{nzt}$ (die weder in OL noch in PD befindlichen Beispiele des KT wurden nicht herangezogen). Dem $o$ vor $i$ in den Einsilbern und Mehrsilbern der Gruppe A) und B), weiterhin vor $\dot{n}$ und $t$ entspricht in allen Südmundarten $o$ (oder in den in Klammern gesetzten Wörtern ein anderer velarer Laut); bei den Einsilbern der (Gruppe C) ist DN., Irt. oi D'T. oi $\sim$ Kond. - $\not i j$ die Entsprechung, bei den Mehrsilbern der Gruppe D) entšpricht dem DN. -oi- $\sim$ DT. $-0 i-\sim$ Kond. oi- am Irtysch mit Schwankungen entweder -oj- oder -oi-, - $u \underset{j}{i}$, ja sogar $a i$. KarJalainens und PaAsonens Aufzeichnungen an der Konda stimmen völlig miteinander überein: dem Kr., Kam. -oi entspricht in PD -oi, dem $o$ vor $n$, $t$ auch ein $o(o)$, den $\mathrm{Kr}$., Kam. - $-\dot{a} i$ hingegen i $\mathrm{m} \mathrm{m} \mathrm{e} \mathrm{r}-\dot{o} \dot{z}-$ !

Hierzu sei noch bemerkt: KARJALAINEN schreibt zwar DN. uōis 'Tier' mit o (OL 94), es wird im Wörterbuch als Stichwort auch genau so angegeben (204b), es kommt in Syntagmen $(67 \mathrm{~b}, 206 \mathrm{a})$ und in Komposita manchmal (206a, b, 575a, 806a) auch mit $o$ vor; in allen übrigen Fällen aber - in Komposita, in Beispielsätzen - ohne Suffix oder suffigiert, mit $o$ (s. 49b, 69a, 135b, 152a, 163a, 239a, 264a, 284a, 284b, 369a, $371 \mathrm{a}, 374 \mathrm{~b}, 417 \mathrm{~b}, 475 \mathrm{~b}, 505 \mathrm{~b}, 539 \mathrm{a}, 566 \mathrm{a}, 575 \mathrm{a}, 612 \mathrm{a}, 623 \mathrm{~b}$, 636b, 702b, 707b, 728b, 815a, 931a, 985a). In der DT.-Mundart hingegen steht dem $u \bar{g} i_{n} 3(204 \mathrm{~b}, 49 \mathrm{~b}, 505 \mathrm{~b}, 633 \mathrm{a}, 717 \mathrm{a})$ nur einmal eine mit $o$ gedruckte Wortform (806a) gegenüber. Aus der Ts.Mundart gibt es Angaben nur mit o (204b, 206b, 417b, 136a, 379a, 505b, 539a, 799b), ebenso aus der Koš.-Mundart (204b, $417 \mathrm{~b}, 806 \mathrm{a})$; in der Sogom-Mundart kann schon ein Schwanken zwischen $o(204 \mathrm{~b}, 806 \mathrm{a})$ und $o(417 \mathrm{~b})$ konstatiert werden; s. noch 


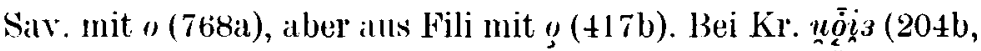
$206 \mathrm{a}, \mathrm{b}, 52 \mathrm{~b}, 67 \mathrm{~b}, 83 \mathrm{a}, 167 \mathrm{a}, 239 \mathrm{a}, 244 \mathrm{a}, 327 \mathrm{~b}, 417 \mathrm{~b}, 539 \mathrm{a}$, $544 a, 575 a, ? 590 a, 632 b, 702 b, 707 b, 806 b, 869 a, 1046 b, 1051 a)$ muss, ebenso wie bei den genau so publizierten Belegen aus der Kam.- (633a) und Boltš.-Mundart (539a) festgestellt werden, dass die Mitteilungen von der Konda ${ }^{1}$ folgerichtig sind (bis auf Tš., $o$ 204b $\sim 417 \mathrm{~b}$ ). Wie viele Abweichungen in den Angaben vom Stichwort (204b) in der DN.-Mundart zu finden sind, so viele Schreib- oder Druckfehler können sicherlich nicht angenommen werden; man könnte eher daran denken, dass - mindestens in der DN.-Mundart -. - Parallelformen mit $o \sim o$ doch existieren. Es verhält sich ähnlich mit dem Wort DN. moi 'Hochzeit', welches und seine Ableitungen mit o (DN. 499a, b, 500a, 871b, Ts. 499a, b) und mit o (DN. 58a, 500a, 737a, Ts. $8 \overline{7} 1 \mathrm{~b}, 1068 \mathrm{a})$ aber Kr. nur mit $\dot{g}(499 \mathrm{a}$, $499 \mathrm{~b}, 500 \mathrm{a}, \mathrm{PD} 1275)$ publiziert worden sind. Vgl. noch $-0 i \sim$ in der nichtersten Silbe, s. weiter unten S. 24.

Tabelle XVI vermittelt den Eindruck, dass sich die Lautverbindung $-o i$ etwa in Einsilbern etwas länger halten kann (wenigstens in den .Jurten am lrtysch) als in Mehrsilbern, wo dem $i$ am Irtysch und an der Konda immer nur palatale Vokale folgen und so durch eine Assimilation aus dem -oi- in Mehrsilbern leicht $>-\ddot{o} i$ - werden konnte.

Wie aus 'Tabelle XVII ersichtlich, ist in den Südmundarten der $o$-Laut vor $\eta$ gut belegt. Der Lautverbindung DN. -o $\chi_{2}(-)$ entspricht in allen Lokalmundarten an der Demjanka, am Irtysch und an der Konda dieselbe velare Lautgruppe, der Lautverbindung DN. -oy- hingegen DT. -oy-, Kond. - $\dot{p} \eta-$ - Auf den palatalen-velaren Gegensatz - $\ddot{\eta} \eta$ : -on weist übrigens KARJALAINEN selbst expressis verbis hin, wenn er bei dem Wort DN. $o_{\supset}^{\prime} \eta$ 'Öffnung der Flasche' bemerkt: "Je nach seiner Stellung tritt dasselbe Wort auf ... mit einem $h$ i n t e r e n Vokal

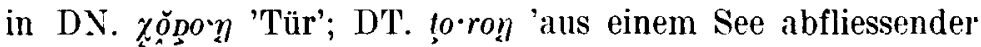
kleiner Bach'» (OL 72).

Ich möchte noch nachdrücklich darauf hinweisen, dass in allen Mehrsilbern mit -ogy- bei KarJaLainex und sogar in fast

1 Vgl. wasion (PD) 3059). 
allen bei PaAsonen in der $\mathrm{z}$ we it e n Silbe a u ch ein velarer Vokal zu finden ist. (Nach den mouillierten Lauten ist, nach dem oben Ausgeführten - s. oben S. 3, Tabelle $\mathrm{V}$ - ein palataler Laut zu erwarten). Ferner sind sämtliche zitierten Wörter - wie aus den Entsprechungen in den Ostmundarten ersichtlich (s. OL 87-109, 276-7, 279-280, 281, STEINITz, GOstjVok. 96) - aus velaren urostjakischen Wörtern ableitbar.

Die Zahl der Wörter mit $o$ in Stellungen, wo nach STEINITZ nur die palatalisierte Variante o erscheinen dürfte, ist in OL und genau in denselben Wörtern bei PaAsonen gross genug (über 20), so kann sie auch STEINITz nicht alle übersehen haben. Er weist auf diese seiner Theorie widersprechenden $o$-Zeichen in einer Anmerkung hin. "Vor $i$ bezeichnet Karjalainen den o-Laut in DN gewöhnlich durch $o$, z. B. Karj. 94 DN $u \overline{o i z}\left(8^{1} \times\right)$, einmal schreibt er aber auch die vordere Variante, also oi (Karj. 74 DN poipe*k 'Schneehuhn')." (GOstjVok. 24, Anm. 45.) Darin hat Steinitz vollkommen recht, dass die Wörter mit DN. -oí(-) (OL 94) dieselben interdialektal entsprechenden Vokale aufweisen wie DN. poipek und die übrigen Beispiele mit DN. $o+$ mouillierter Konsonant (OL 74); hierauf hat übrigens schon Wicirmaxn (FUF A IV 11) hingewiesen, ohne zu bezweifeln, dass KarJa LaINen -oi(-) bzw. -oit(-) richtig geschrieben hat. Wic aus dem Wörterbuch nachweisbar ist (s. oben S. 19-20), liegt wahrscheinlich bei KARJALAINEN bei mehreren Wörtern ein Schwanken DN. -oij(-) $\sim-0 i(-)$ in den Aufzeichnungen vor, es wäre m. E. jedoch allzu gewagt, alle Wörter mit DN. -oin(-), - - ja sogar mit DN., Ts., Koš., Fil., Kr., Kam. -o $i \sim$ PD -o $i$ als "gelegentliche Abweichungen und In k on se qu en z e n in der Bezeichnung kombinatorischer Varianten" (GOstjVok. Anm. 32, vgl. Anm. 45) zu betrachten. Kar.JALainen hatte ja ein phonetisch gut geschultes Ohr, in OL stand gerade der Vokalismus der ersten Silbe im Mittelpunkt seines Interesses. Ausserdem handelt es sich um Wörter, die aus velaren urostjakischen Wörtern herleitbar sind $(* a)$, ferner gibt es vor mouillierten

1 Genauer: $o$ ist OL S. $945 \mathrm{mal}$ in Wörtern aus DN. (s. jedoch noch je ein DN. -oi OL S. 88, 99, 107), 3mal von der Konda und einmal (x̧oi- 'Ireffen') aus DT., Kond. belegt. 
Konsonanten Aufzeichnungen mit DN. o, ohne dass dieselben Wörter auch mit o zu finden wären. Bei den Wörtern mit -oi- $\sim$ -oi- können wir nicht beweisen, dass sie genau aufgezeichnet worden sind, wir können nur stets im Beispielmaterial des Wörterbuches ein Schwanken oi $\sim \underset{\sim}{i}$ in der ersten und nichtersten Silbe konstatieren (s. S. 19-20, 24) und KaRJaLAINEN entweder Vertrauen schenken oder nicht (in den nach -oi- folgenden Silben sind palatale Vokale zu erwarten, s. oben S. 3). Bei den Wörtern, wo vor $\eta o$, und nicht $g$ steht, $\mathrm{k}$ a n $\mathrm{n}$ j e d o c h b e$\mathrm{w}$ i e s e $\mathrm{n}$ werden, dass das o $\mathrm{r}$ i $\mathrm{c}$ t $\mathrm{i}$ g ist. STEINitz meint: "In zwei Wörtern steht Karj. 108-9 DN on, aber schon DT regelmässig on; es handelt sich zweifellos nur darum, dass Karjalainen die kombinatorische Variante $o$, die i. A. vor $\eta$ erscheint, hier gerade in DN nicht beobachtet hat (weshalb er dann auch $\eta$ schreibt; ...)» (GOstjVok. Anm. 45.). In diesen Fällen bezeugt aber der velare Laut der zweiten Silbe die Velarität der ersten Silbe (DN. pōnâm-, DN. ōnân't'-) bzw. in den übrigen Dialekten die Palatalität der zweiten Silbe die Palatalität der ersten Silbe (Kr.

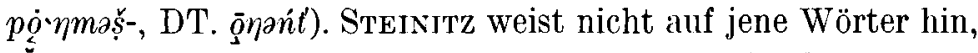
die in OL nur aus der DN.-Mundart (ohne palatale Entsprechungen in anderen Südmundarten) mitgeteilt wurden. Wie aus dem Wörterbuch und Tabelle XVII ersichtlich, haben auch die übrigen Südmundarten in der Lautverbindung -on $\left.\chi_{-}^{-} \sim-o \eta\right)_{-}^{-}$ den velaren $o$-Laut bewahrt (vgl. Kond. mo $\eta \psi_{\kappa} \hat{\partial} s^{-}$velar, aber

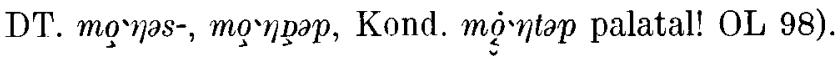

Einerseits konnte also bewiesen werden, dass $o$ vor $\eta(\eta \chi \chi)$, $n^{\prime}, t^{\prime}$ und $i$ vorkommt, andrerseits sollen jetzt Beispiele angeführt werden, wo [ö] nicht vor den von STEINITz erwähnten Konsonanten erscheint, sondern vor anderen.

Aus OL kann nur e i n Beispiel zitiert werden, wo o nicht neben $k$ oder vor mouilliertem Konsonanten oder $\eta$ steht: DT. sōlak 'mit dem Strome treibendes Eis', vgl. Kond. sū l̨ ebd. (OL 75, 78, 854a). ${ }^{1}$

Zwei der im Wörterbuch befindlichen Beispiele mit $g$ aus der DN.-Mundart scheinen auf einem Druckfehler zu beruhen:

1 In DT. sol'Gan Adj. (854b) ist $l$ sicherlich gleichwertig mit $l$, vgl. Tolvonen, FÚF XX, 80. 


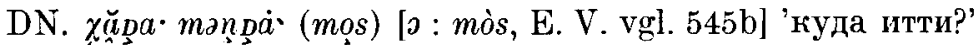
(366b); DN. sòtpây k[ơ sōpấp 'Schote (des Pfeffers)' (885b), woraus mit dem Ablciter $-\eta$ * sòtpâ $\eta \mathrm{zu}$ erwarten ist (vor $\hat{a}$ der zweiten Silbe ist auch in der ersten ein velarer Laut zu erwarten). Ein unanfechtbares Beispiel

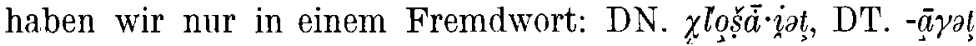
'клещи | Zange' (383b).

An der Konda erscheint $o$ jedoch oft auch in anderen phonetischen Umgebungen als in den von STEIxitz umschriebenen:

Kr. $\stackrel{i}{\sim} u$ 'was? hallo! (fragt man, wenn der Redende weit weg ist und ruft)' (15a);

Kond. $p \underset{z}{u} u$ 'Zapfen (ausser der Zeder)' (OL 15; 663a, PD 1914);

Kr. pg̣in ùtṣ̆ uarät tēkant 'wenn (ein) Kleid voll Blut wird' (978b);

Kr. $p \grave{g} m p \bar{u} \chi \hat{\partial} t$ 'Sargatische Jurten (dort wohnte früher $p[\overline{\grave{s}} m 3]$ = russ. Фома, Name)' (704b);

Kr. klipppan '(Eisen)blech' (469a, PD 778);

Kr. pọps, Loc. pọ panз 'Bär (zu den Kindern, Kinderspr.)' (718b);

Kr. tăx $2 \hat{\partial} t$ pist rat (unübersetzt, etwa Druckfehler?) (814a);

Kam. ¿̨pt[o·rnikns] 'am Dienstag' (20b).

Die hier mitgeteilten i-Zeichen kommen also in der Kr.Mundart bis auf eine Interjektion neben $p$ vor, vgl. noch bei PaAsonen: pòlà 'Acker' (1907), pómats 'Arbeitsfest' (1908),

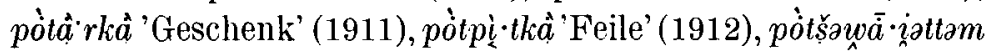
'bewirten' (1913); anderswo, auch in einem Worte fremden Ursprungs: ’̀trüup 'Kleie' (1587); in einer Aufzeichnung aus einer anderen Lokalmundart: Ts. $s \overline{\bar{o}} \cdot-p \dot{u} t$ 'tönerner Topf' (PD 2256).

Die mit $\dot{o}$ geschriebenen Varianten des [ö]-Lautes im KT erfüllen alle die von STEINiTz festgestellten Bedingungen; zwischen zwei mouillierten Lauten:

Koš., Sogom tơnt', Kr. tónț ${ }^{1}$, aber DN. tơnt', Ts. tơnt' 'Schnee' (1117b, PD 973) DN. tón't' (894a, mit Suffixen 512b, 680a, 728b, 1024b, DN., Kr. $\dot{o}$ 822a, 936b, Kr. 488a, 654b), Ts.

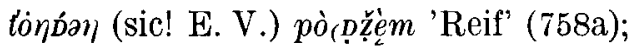

1 Aber auch Kr. tọnt $93 a$. 


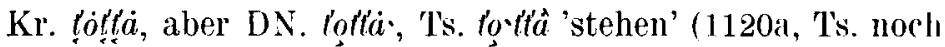
802b, 866b; PD 971, mit $\dot{0}$ : DN. 462a, 636b, 815a, 931a, 944a, D. 899b, DN., Kr. 254b, Kr. 11a, 28a, 137b, 569b, 748a, 944b. 1018b, 1043b, 1068b, Sav. tót 327a);

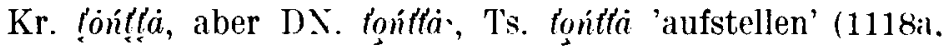
PD 974), s. Ableitungen 1118b, auch Ts. mit $\dot{o}$ (379a); vgl.

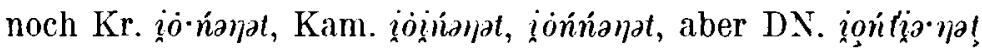
'altertümlicher "Näh»korb der Frauen' (176a).

Nicht nur zwischen zwei mouillierten Lauten:

Ts, Kr. tón, DN. tơn, Kam. tọn 'Ort, wo das Netz allsgeworfen wird' < тоня (1006a, PD 2635);

Kr. tón, DN. toń, T's. toń, DT. ton 'Lein, Flachs' (1077b, PI) 2634) aber DN. tòn (731b);

DN. lak 'tie' (155b) aber sonst log $\%$ ( $42 \mathrm{~b}$ und noch cin dutzendmial mit o, s. unten S. 65).

Auch in der nichtersten Silbe kann man in den Südmundirten einem [ö] begegnen, hier unter den von STELNiTz festgelegten Umständen und neben $p$. Es ist nur in einem oft gebrauchten Wort belegt: DN. mopōiz, Fil., Ts. maDōiz, Kr.

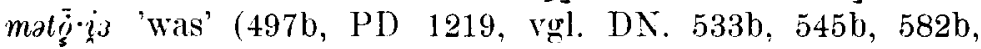
DN., Kr. 763a, 1030b, Kr. 216a, 235a, 454b, 580a, 825a, Kr.,

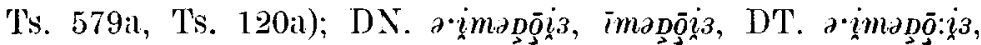

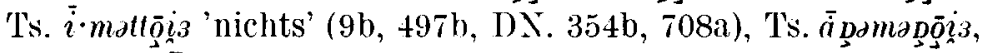
Kr. ăhtâmatịiga 'etwas' (102a), Kr. matịịa 'warum' (497b); bei

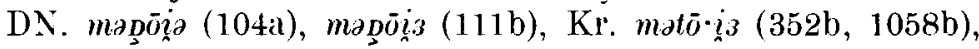
matơ $(547 \mathrm{~b})$ und wahrscheinlich auch bei Sar. meooi (883a) sind die $o$, bzw. $\dot{\dot{g}}$-Zeichen wohl Druckfehler statt $o$,

In den meisten Beispielen folgt dem [ö] auch in der zweiten Silbe ein $k$ oder $i$ :

Tš. ikig u 'Kumt' (24a, PD 190);

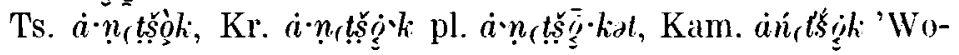
gule' (55a, PD 81);

Kr. $u \dot{a} \cdot l, j_{\xi}$ 'Wäschbleuel' (223b, PD 2892);

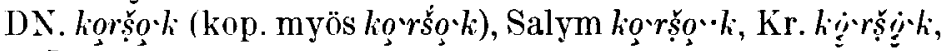
kijers

DN. nasmò ka, nasmọká < насморка (597b);

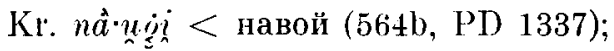

DN. si-rig? < синій (863a); 


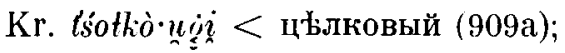

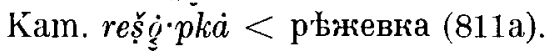

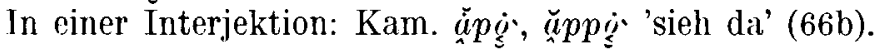

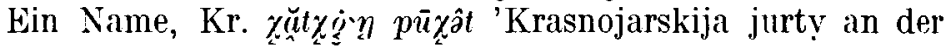
llündung des $\chi\left[a \cdot \chi_{z}\right]$ i[ $\left[e \chi_{3}\right]^{\prime}$ (363a), scheint ein Kompositum aus $\chi a t \chi 3$ und Kr. zu sein. Wahrscheinlich ist Ts. tṣ̆òožò skija' (961a) auch so zu beurteilen: 'țşoțtş-Mündung', obwohl ein

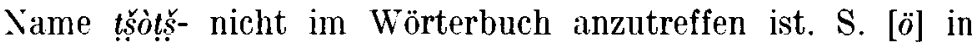

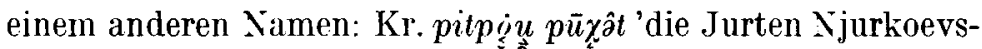
kija' (769b).

Ts. pŏrgo'n 'Schnur der Laufangel' (731b) scheint ein Kompositum zu sein, obwohl weder das erste noch das zweite Glied allein belegt ist.

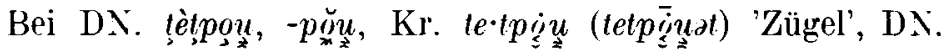
tètpoup Laut auch in der DN.-Mundart neben $p$ und vor $u$ zu sehen (s. jedoch Ts. tètpou ebd.).

Vgl. noch unten S. 30, $\sim \sim[\ddot{o}] \sim[\ddot{o}]$ in der zweiten Silbe bei DN. tŏgoi usw.

Da Kr. lọgrạpia entscheiden, ob hier ein Kompositum oder eine Ableitung in der zweiten Silbe einen [ö]-Laut hat.

In einem fremden Namen, Kr. iaraugid 'Jurovskoe' (182b), steht nach zwei velaren Silben in der dritten Silbe ein [ö].

Aus PaAsoness Aufzeichmungen kömnen noch die folgenden Beispiele hierhergezählt werden, neben $k$ in Fremd- oder Lehnwörtern: rè̀tkò. 'selten' (1994); sâmò่k 'Hahn (an der Flinte)' (2112); vgl. noch vor $i$ : rèmò่ $i$ 'vielleicht' (1990).

Aus meiner Zusammenstellung ergibt sich, dass [ö], hauptsächlich an der Konda, auch neben $p$ zu finden ist, ausserdem ist je in einem Beispiel DT. sol-, bzw. DN. DT. -loşs-, Kond. ôt-, Ts. sio $\bar{o}$ zu sehen. Wichtig ist, dass die [ö] enthaltenden Wörter (ausser einer Interjektionsvariante und einem fremden Namen) in den übrigen Silben auch n u palatale La ute aufweisen, vgl. hierzu oben $\mathrm{S} .22-5$ und s. auch noch die angegebenen Belegstellen zu den Tabellen XVIII- XX. Es gibt sogar pa-

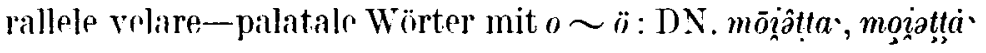


'zu Besuch sein' (499b) usw. Eigentlich wurde zu KarJalainens Zusammenfassung nur sehr wenig hinzugefügt, er meint nämlich $» \mathrm{DN}$. o [begegnet] in relativ wenigen Wörtern, von denen die grössere Hälfte noch fremden Ursprungs ist. In e i $\mathrm{n} \mathrm{h} \mathrm{e} \mathrm{i-}$ $\mathrm{m}$ i s c h e $\mathrm{n}$ Wörtern folgt auf das $g$ stets ein $k$ oder $\eta$ (ausser in dem DT. Worte sōlak), in einigen Fällen ein mouillierter Konsonant (in einem Worte $i$ ); in den L e h n w ö r t e r n geht gewöhnlich ein $k$ voran, das in den südlichen Dialekten... $\mathrm{n} u \mathrm{r}$ vor vordere $\mathrm{n}$ okale $\mathbf{n}^{\mathbf{1}}$ angetroffen wird. Es leuchtet folglich ein, dass das Auftreten des $o$ von den ihm benachbarten Konsonanten abhängig ist” (OL 78). KARJALAINEN hat in seinem Werk die Mundarten an der Konda nicht besonders beachtet, so blieb bei ihm Kond. ig neben $p$ unerwähnt.

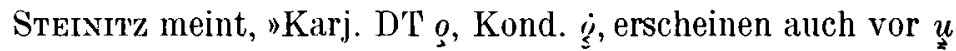
$[=w]$ (Karj. 14, 15), vor dem o nicht vorkommt" (GOstjVok. 24). Hierzu sei bemert: bei D'T. kouram 'heiss' (OL 14) steht [ö] neben $k$, wo es auch sonst häufig vorkommt, bei Kond. pigu 'Zapfen' (OL 15) neben $p$, wo es, wie aus den Tabellen XVIII $-\mathrm{XX}$ ersichtlich, auch nicht selten ist. Die Lautverbindung DT., Kond. -ow- ist zwar nicht häufig, sie existiert jedoch: Kr. owto•rńiknз 'am Dienstag' (20b), Kr. touñärâta 'ausspannen' (973a); aus der nur mangelhaft bekannten DT.-Mundart kenne ich kein Beispiel.

4.4. Den reduzierten $\ddot{o}$-Laut $[\tilde{o}]$ finden wir im Wörterbuch von Karjalaines folgendermassen: DN., Ts. $\check{o}, \mathrm{Kr}$, Kam. $\stackrel{\check{g}}{\text {, }}$ manchmal DN. $\check{\rho}, \mathrm{Kr} . \breve{\rho}^{2}, \stackrel{\breve{g}}{g}$. In den Beispielsätzen des Wörterbuches erscheinen einigemal Wörter mit [ü] (d.h. mit DN. $u, \dot{u}, \dot{u}$, Ts. $u$, Kam. $u$ ), die als Stichwörter mit [ö] mitgeteilt wurden; z. B. DN. kŏgr, Kr. kŏr 'Fuss', DN. kŏr re $\cdot m, \mathrm{Kr}$. kŏrem '[mein Fuss]' (418b), aber Kr. kur (169b), DN. kurè’m, Kr. kurem (736a). Interessanterweise ist in den Texten überhaupt

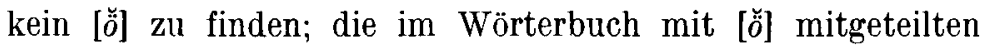

1 Sperrung von mir, E.V.

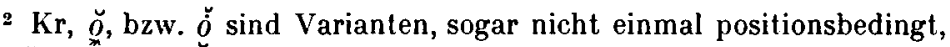
s. kŏlapit, kợla $p\left[i^{\cdot} t\right]$ ] '[Gefährte]' (400a, 779a); kŏ́rnik < курникъ (433a),

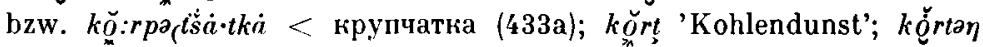
Adj. (433b) usw. 
Wörter wurden in den Textaufzeichnungen mit DN. $u, \dot{u}, \dot{u}$, Ts. $u$ geschrieben, über die unterschiedlichen Bezeichnungsweisen des [ö] in der Kr.-Mundart s. Abbildung 1; vgl. sogar im Wörterbuch Kr. nŏglat bzw. numàt '[dich]' (240a, 516b, 714b, bzw. 553b, 912a; 556a, 610a). Das Verhältnis der [ö] : [ï]-Laute (oder der Bezeichnungsweisen) erfordert noch weitere Untersuchung. Da aber KaRJalainex selbst über DN. .̆ , Kond. ŏ schreibt (OL 78-87), muss es unbedingt einen solchen Laut geben, obwohl Stersitz seine Meinung darüber so zusammenfasst: "Der reduzierte $\breve{o}$-Laut wird von $\mathrm{K}$ a r j a $\mathrm{l}$ a i n e $\mathrm{n} \check{o}$ geschrieben ..., ausser neben den Palatalen $k, \eta$, in welchen Stel-

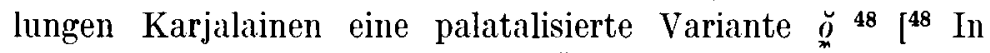
Kond. schreibt Karjalainen auch $\stackrel{0}{0 .]}$ bezeichnet... Das Verhältnis Karj. $\stackrel{o}{o}: \stackrel{o}{o}$ entspricht also genau dem Verhältnis Karj. $o: o$. P a a s o n e n schreibt $o$, die palatalisierte Variante neben $k, \eta, w$ bezeichnet er aber mit $\dot{o}$. . Paas. Kond. $o-\dot{q}$ entspricht also genau Karj. DN $\check{o}-\breve{o}$, nur dass in DN die palatalisierte Variante nicht vor $w$ erscheint» (GOstjVok. 24, vgl. GFgrVok. 68, Anm. 12); "Paas. Kond. o kommt ... vor $w$ nicht vor ${ }^{52}$ [ 52 ausser nach $\chi$ : Paas. $\chi o u$, 'lang' 1 Nach $\chi$ kommt a nicht vor.]" meint STEINiTz (GOstjVok. 26).

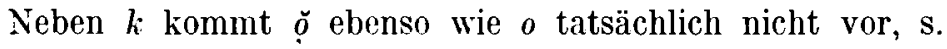
unten S. 34; vor $\eta$, wo $\underset{m}{\circ}$ gut belegt ist, ist der $\check{o}$-Laut sehr

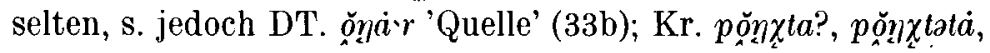
Kam. pŏnxtatá 'im Wasser aufschwellen' (693a); Kam. sŏnxरr 'Suppe' (851b, PD 2230); DT. tó. $\eta \chi_{\alpha} e m$ 'am Boden angebrannt'

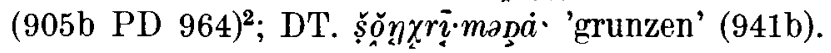

Die Schreibweise - ŏ $u$ - ist bei KarJaLaInes in OL und auch im Wörterbuch in der DN.-Nundart und in den übrigen Südmundarten keine Seltenheit:

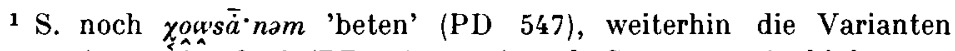
iowan, iaowan 'nachts' (PD 359, 334), vgl. STEINITz, "Archiphonem"

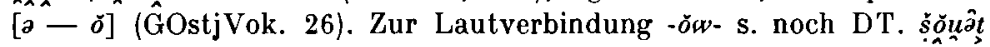

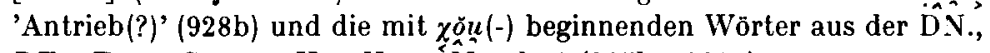
DT., Ts., ? Sogom, Kr., Kam.- M̂undart (285b-290a).

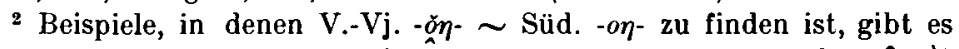

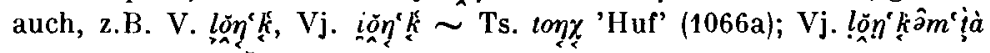
$\sim$ DT. to'ngG ìmațtiv 'klopfen' (482a) usw. 


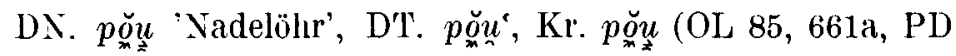
1915, vgl. 1920);

DN. pŏ

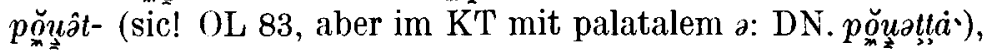

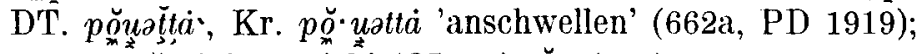

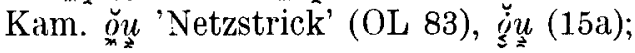

s. noch D'T. pŏual 'Hechtdarm' (661b, ?PD 1916);

Kr., Kam. ל厶ukamtta 'eine Ohrfeige geben' (20a);

S. bei PaAsonex pojw 'Zapfen' (1914), pojworà $k$ 'Eierkuchen' (1918).

Steinitz, der sonst die bei KarJalainen mit $\underset{\sim}{\not}$ bezeichneten Laute als neben $k$ und $\eta$ vorkommende palatalisierte Varianten des $\check{o}$-Lautes betrachtet, meint, obwohl die aus OL eben zitierten Wörter, und zwar alle drei, Entsprechungen mit $\breve{o}$ in den ()stmundarten haben, dass "zwischen $p$ und $u$, wo also dic Labialisierung des reduzierten Vokals besond̄ers stark ist" (GOstjVok. 25), ŏ w wnur eine kombinatorische Variante von [aw]» (Anm. 51) sei; seine Begründung hierfür ist, dass bei KarJalaninex vor $w$ auch DN. ŏ zu finden sei (ebd.). Darin hat S'lEINITz unbedingt recht, dass $\underset{x}{\not{o}}$ hier nicht die Variante von $\breve{o}$ ist (meiner Ansicht nach nirgends), ob aber -ŏ $u$ - immer als [-au-] aufzufassen ist, soll näher untersucht werden.

Die Lilutverbindung påu- kommt bei KaRJaLAINex tatsächlich nicht vor; $-a ̊ u$ ist aber auch im Wörterbuch, nicht nur in OL $(205-8)$ zu finden:

DN. reuattia 'mischen' (793b)', wo dem DN. 口 Fil., T's., Kr. d, aber V., Vj., VK. бo, 'Trj., Mj. a entspricht; eine andere Auf-

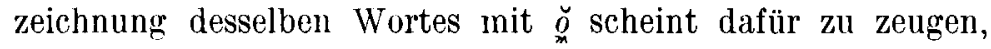
dass man hinsichtlich dieser Lautverbindung die Meinung von

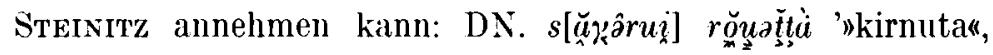
pieksää voita' (839a, PD 2034).

Vgl. noch Kam. tŏuar 'morsch' (972b, PD $2654 \sim \mathrm{Kr} . ə \sim$

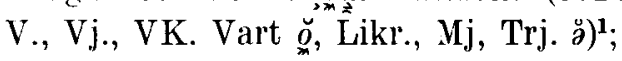

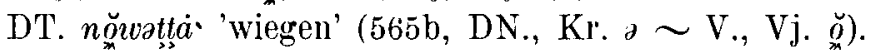

DN. tşo ợ (928a) zengt auch hierfür (obwohl in der V.-Mundart ein ä steht).

1 Vgl. Steinitz, op. cit. S. 95 und Anm. 236, 
Eigentlich äussert sich gewissermassen KarJaLaIneN selbst im Sinne der Meinung von Steinitz, indem er schreibt: »dass PaAs. aow hier [in "Kond. se ow whä̈n ${ }^{1}\left[{ }^{1}\right.$ Wahrscheinlich Druckfehler für *soowkmằn $\left.{ }^{1}\right]$ mein $*_{\breve{o}}$ repräsentiert." (OL 86); "PAASONEN schreibt $\ddot{\theta}$, in einigen Wörtern əou" (OL 78, Anm.).

Weitere Beispiele mit $\stackrel{\circ}{⿲ ㇒}$ in einer labialisierenden Umgebung sind: DT. pŏ Wörterbuch DT. puвs, ривз [718b]).

Bei DT. pờm, DN. pam 'Dampf' (OL 189) meint KarJALAINEN: "Sicher muss wohl der Labialvokal... auf den Einfluss der ihn umgebenden labialen Konsonanten zurückgeführt werden" (ebd.), eben damit erklärt er das Zustandekommen des [ั̆] aus a und behauptet, dass das $\check{\theta}$ keine Variante von $a$ ist (rgl. Steinitz, GOstjVok. 114).

Vor $m$ s. noch $\underset{\sim}{\circ}$ in DT. tơmmbì! 'Kelle von Birkenrinde' (OL 69, 123, s. 911a), hierzu gibt es eine bis zum Ende velare Variante: DT. tŏmba? (911a), bei Kr. tŏmpal (911a) scheint die Pluralform, Kr. tợmpä.lât darauf zu deuten, dass im Sing. das Kr. ŏ zu korrigieren ist.

Wenn wir auch annehmen, dass -ŏu- nach $p^{-}$, oder sogar überall eine Variante von - $\jmath_{o} u$ - ist und dass etwa a neben $p$ oder $m$ auch labialisiert werden kann und $\breve{o}$ in diesen Fällen auch nur eine Variante zu a darstellt, so bleiben jedoch noch einige Aufzeichnungen mit $\underset{x}{\breve{x}}$ übrig, wo dies gewiss nicht der Fall sein kann, nur bei DN. uox $\gamma$ - 'schreien' (OL 83, 217ab) könnte vielleicht noch dem Einfluss des anlautenden $w$ Rech-

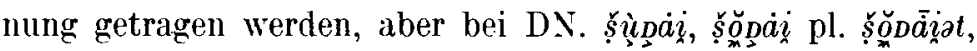
şapga'i 'Haselhuhn' (964a) kann die Labialisation keinem der benachbarten Konsonanten zugeschrieben werden. S. noch den $\breve{o}$-Laut in einer nicht labialisierenden Umgebung:

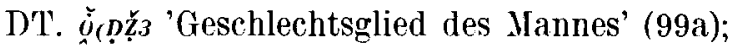

D'T.

I'T. ŏ

Kam. koll 'kupferner Knopf' (907a);

Kam. เ⿳̆口口 rop 'Schrot' < дробь (1039a) zeugt dafür, dass die Kam.-Mundart einen ŏo-Laut kennt, durch den sogar Konsonan-

1 S. PD 2180 mit $\partial_{6}$. 
tenhäufungen aufgehoben werden können. Kond. sọmat 'Birke' (2252) ist bei PaAsonen zu sehen.

Vor $i, \dot{n}$ und t's kommt $\underset{x}{o}$ zwar auch vor (STEIsitz weist nicht auf diese Umgebung hin), aber nur nach $k$, so konnten diese Stellungen bei Steisitz doch mit Recht unerwähnt bleiben; auch vor $t$ ist es im Wörterbuch belegt (in OL nicht): DT. ŏtl'au 'Pulvermass' (133b). Bei PaAsonen: $o k_{\alpha}^{\prime}-m \bar{o} \cdot \chi^{\hat{3}}$ 'Tochter der älteren Schwester meines Vaters' (1578). Es sei nebenbei bemerkt, dass vor $i$ und vor palatalisierten Konsonanten auch ŏ erscheint, um Beispiele n u $\mathrm{r}$ aus OL zu zitieren: DN. xŏjam- 'fallen, sich vermindern (vom Wasser)' (109), DN.

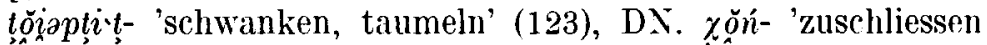
(die Augen)' (115).

In einigen Fällen ist das Zeichen $\underset{x}{\circ}$, bzw. fehler:

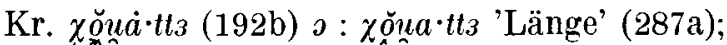

DT. sớttta 'sich abspiegeln' (891b), aber mit o: sòDo ! (ebd.);

DN. tŏm $(109 \mathrm{~b}, 697 \mathrm{~b})$ ॰ : tŏm 'jener' $(975 \mathrm{~b})$;

Kr. ńärə-tŏxmât 'bloss, nackt' (631b) ə : Kr. nära-tŏmât id. (1072b, wo $n$ - in n- zu korrigieren ist); diese dürfen also nicht zu unserem Beweismaterial gerechnet werden. ${ }^{1}$

In einem Fall müssten die Originalaufzeichnungen des Wörterbuches untersucht werden, bevor man irgend etwas über Schreib- oder Druckfehler oder Schwankung in der Aussprache aussagen könnte; hier erscheint [ö] als eventuelle Variante in

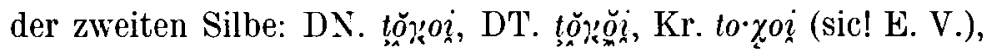
tơxgi (sic! E. V.), Kam. toxei 'weg; hinaus' (975ab, vgl. Kr. to భ0o 476a, Kr. tơxị 152a, 526a, 526b, 527a, 528a, 639a, 801a, 961b, 1047a); rom Stichwort abweichende Mitteilungsweisen sind noch: DN. to 526a, 526b), ja sogar DN. tơ $\gamma_{\alpha}^{\circ} i(19 \mathrm{a} ; 151 \mathrm{~b}, 423 \mathrm{a}, 527 \mathrm{~b}, 662 \mathrm{~b}$, 731b, 732a, 961b, 1024b, 1029a) und DN. to $7 \gamma_{3}^{\circ \cdot i}$ (595b); s. noch

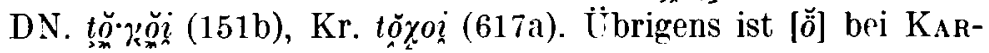

1 DN. sŏk 'eine Art Zierat aus Blei' (OL 82) ist hinwieder in sŏk zu korrigieren, wie es auch im Wörterbuch (833b) aufgenommen wurde; hierfür spricht, dass es bei $\mathrm{DN} . \breve{o}$ in $\mathrm{OL}$ eingereiht wurde, weiterhin kommt $k$ nur neben palatalen Vokalen vor (s. unten, S. 34). 
JALAINEN in der zweiten Silbe nur noch einmal, als Variante von [ö] belegt: DN. tètpou, -pŏu 'Zügel' (1032b, s. PD 2496), bei PAASONEN auch noch in Wörtern fremden Ursprungs: lokơn

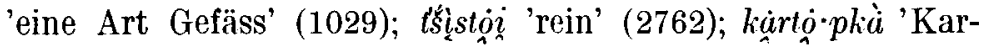
toffel' (667).

Wie aus den angeführten Beispielen ersichtlich, erscheint $\stackrel{0}{x}$ nicht nur neben $k, \eta$ und neben $p$ und $w$ (wo es etwa als Variante von a betrachtet werden kann), sondern auch vor $l, m, r, t s, l$ und $t$ in Wörtern, wo vor dem [ö] kein $k$ oder $p$ steht. Aus den Beispielen ist ferner ersichtlich, dass alle Wörter in der ersten Silbe mit $\underset{\sim}{\circ}$ in den übrigen Silben $n u r \quad p$ a lat a le Vokale haben, s. Tabellen XXI-XXIII und die Belegstellen im Anhang.

M. E. ist die Frage, ob die ziemlich seltenen Vokalzeichen $\stackrel{\emptyset}{\varrho}$ bzw. $\breve{x}$ selbständige Laute oder Varianten der Laute $o$, $\check{~}$ repräsentieren, nicht ausschliesslich mit Hilfe der umstehenden Konsonanten, sondern eher, ebenso wie bei dem $q$, durch die nachfolgende Silbe zu entscheiden. In den nichtersten Silben der Wörter, die in der ersten Silbe $\stackrel{0}{\circ}, \stackrel{0}{o}$ haben, sind immer nur palatale Laute zu sehen, in denen, wo aber in der ersten Silbe $o$ oder $\check{o}$ steht, stehen velare (bis auf die Fälle, wo ein inlautender palatalisierender Konsonant die palatale Fortsetzung hervorruft). Nach meiner Auffassung müssen diese Wörter nicht, sie dürfen sogar nicht als Druckfehler betrachtet werden, die STEINITz (GOstjVok. S. 24-5, Anm. 32, 45, 51, 69) als Wörter mit "gelegentlichen Abweichungen . . . in der Bezeichnung der kombinatorischen Varianten" (bei KarJalainen und bei PaAsonen oft in denselben Wörtern!) betrachtet, - weil diese nur so in seine Theorie hineinpassen. Es sind keine Druckfehler, sie passen mit ihrem Vokalismus ja ohne Zwang in das System der südostjakischen Vokalharmonie gut hinein. Hätte STEINitz nicht nur die konsonantische Umgebung der Vokale, sondern auch das Verhältnis der Vokale der ersten und der übrigen Silben ins Auge gefasst, dann hätte er sicherlich nicht die Existenz der

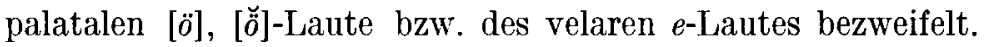
Dazu hätte er aber von KaRJALAINes mehr als nur das schon damals erschienene Werk, OL, g u t kennen müssen.

4.5. Angenommen, dass das urostjakische Vokalsystem genau 
dieselben Laute enthalten hat, wio sie KaRJaLAINEx um dir Jahrhundertwende am Vach und am Vasjugan noch angetroffen hat (()L 276-89, jedoch unbegründet und überflüssig mit $\varepsilon$, $\dot{e}$ und $\check{a}, 1$ Wichmaxx, FUF A IV, 11 Anm.; Steisitz, GFgrVok. 64; GOstjVok. 2, 28, 48, 96-7), dann können die @, [ö], [ö]-Laute des Südostjakischen zwanglos, ganz logisch aus diesem urostjakischen Vokalsystem direkt abgeleitet werden, mit wenig Ergänzungen fast genauso, wie KaRJaLAINex es getan hat, natürlich ohne das von STEs.stz rekonstruierte Vukalsystem der Westdialekte (GOstjVok. 52, 53, 55).

Der velare $i$-Laut ist in der Mehrzahl der finnisch-ugrischen Sprachen verschwunden, demgegenüber hat das $i$ im Ungarischen und auch im Finnischen die Möglichkeit, in palatalen und in velaren Wörtern vorzukommen, ohne dass dadurch die Vokalharmonie gestört wäre. Es ist mun wirklich kein Wunder, dass das urostj. *i nur in einem Teil des ostjakischen Sprachgebietes, nur im Osten erhalten geblieben ist; im Westen ist es in der ersten Silbe mit den aus urostj. ${ }^{*} i$ herleitbaren Lauten zusammengefallen, bis auf in Wörtern mit $\%, \eta, \eta$ im Auslant der ersten Silbe. Diese Laute konnten nämlich die Velarität der ersten Silbe bewahren, und so entstand vor diesen Lauten aus dem * $i$ nach dem Aussterben dieses Lantes ein anderer velarer Laut $\xi$; stand das $*_{i}$ nach einem anlautenden $\%$, dann entstand ein $e$, welches wetwas diphthongisiert ausgesprochen wird" (OL 174, Anm., vgl. PD 460-- 473 Wörter mit zèęe-). Für die erste Silbe hat KarJalainex die Entwicklung der aus urostj. ${ }^{*} i$ herleitbaren Vokale höchstwahrscheinlich richtig dargestellt (OI, 283-4).

In der nichtersten Silbe konnte aus $*_{i}$ auch ein $i$ werden, und wenn das Wort noch weitere Silben hat, so folgt nach dem $i$, ebenso wie nach gewissen "palatalisierenden" Konsonanten, ein palataler Laut; das in nichterster Silbe stehende $i$ der velaren Wörter kann jedoch noch zur Zeit der Jahrhundertwende seine Velarität bewahrt haben (in ganz seltenen Fällen sogar in der ersten Silbe, s. oben S. 15-8), aber auch in diesem

1 Urostj. $\check{\emptyset}$ und $\check{\emptyset}$, bzw. $\breve{o}$ und $\check{o}$ stellen eigentlich auch bei $\mathrm{K}_{\mathrm{ARJA}}$ LAINEN nur bedingt zwei verschiedene Laute dar (OL 286, 287-8). 
Fall komnte das Wort, falls die $i$-Variante des $i$-Lalltes in der nichtletzten Silbe stand, palatal fortgesetzt werden.

Mit den [ö]- bzw. [ö]-Lauten verhält es sich gewissermassen ähnlich. "Ein urostjakisches $\dot{\imath}$ lässt sich nur in einigen bestimmten Fällen - in der Nachbarschaft von $k, \gamma$ und $\eta-$ feststellen" (OL 278), vgl. "Urostj. *ö ist nur neben den Palatovelaren ${ }^{*} k,{ }^{*} \eta,{ }^{*} \gamma$ nachweisbar» (STEINIT' GOstjVok. 73, vgl. 75), und nach Meinung beider wurde im Südostjakischen ein $e$ daraus (ebd.). Bei urostj. * $: ;$ und $*_{\tilde{o}}$ wird keine phonetische Umgebung angegeben (OL 278, 286, Steinitz, op. cit. 62, 94), aber in der Geschichte des finnisch-ugrischen Vokalismus steht:

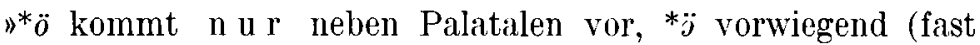
ausschliesslich) neben solchen" (75). "Dass dic Labialität des Vokals [urostj. $\dot{g}(o)$ ] in einigen Fällen auch in den westlichen Dialekten bewahrt ist, ist dem Einfluss der $u \mathrm{~m} g$ e b e $n \mathrm{~d}$ e $n$ Konsonanten zuzuschreiben" meint KarJaLaines (OL 278), und auch Stersitz ist der Meinung, dass meben $k$ und $\eta \ldots$ es [*ä] seine Labialität bisweilen ... erhalten» hat (GOstjVok. 62); ich kann ihm aber darin nicht mehr beistimmen, dass es mit den "hinteren labialen Vokalen zusammengefallen" sei (ebd.), weiterhin meint STEINiTz: "neben $k$ und $\eta^{237}\left[{ }^{237}\right.$ In den Belegen kommt fast nur *ö̀ neben $\eta k$ (nicht neben $\eta$ ) vor.] hat urostj. * $\breve{a}$ auch in den west. Dialekten seine Labialität beibehalten" (GOstjVok. 98, vgl. GFgrVok. 26), was aus den Beispielen s. S. 28-31, 62-70 klar ersichtlich ist, aber, dass "es in Irt. mit dem entsprechenden hinteren labialen Vokal $\check{o} . .$. zusammengefallen» wäre (GOstjVok. 98, GFgrVok. 26), kann man nicht mehr bejahen.

Nach KarJalamess Meinung sind nicht alle heutigen südostjakischen vollen und reduzierten $\ddot{o}$-Laute aus palatalen urostjakischen Lauten (OL 278, 287) ableitbar: vor $\eta$ ist, seiner Meinung nach, aus ${ }^{*} a,{ }^{*},{ }^{*}{ }_{a}$ bzw. ${ }^{*} \check{g}$ ein volles bzw. reduziertes $\ddot{o}$ geworden (OL 277, 280, 281, 287). Hierzu sei bemerkt, dass $\eta{ }^{\prime}$ die Velarität des davor stehenden Vokals in allen Mundarten bewahrt hat, s. oben S. 20. Aus urostj. ${ }^{*} a$ wurde auch vor mouillierten Konsonanten DN., DT. o, Kond. $\dot{g}$ (OL 277). Diese. Vokalentwicklung kann mit Hilfe der Regel der südostjakischen Vokalharmonie begründet werden: nach dem 
mouillierten Konsonanten wird das Wort palatal fortgesetat; zur Zeit, als diese Regel noch neu war, wollte das Sprachgefühl wohl die alte, bis zu den heutigen Tagen in den V.-Vj.-Mundarten geltende Regel der Vokalharmonie befolgen, und mit einer regressiven Assimilation die erste, velare Silbe des Wortes den übrigen palatalen Silben assimilieren. Eine ähnliche Erklärung kann auch bei den Fällen gegeben werden, wo nach dem urostj. ${ }^{*} a$ ein $i$ gefolgt war, in diesen Fällen sei, nach KARJ LAINEN nur in D'T. und Kond. - bis auf Wörter, die mit $\chi$ oder $s$ - anlauten (OL 94) $-g, \dot{q}$ zustande gekommen, in DN. nicht (OL 277). Aus dem Belegmaterial der OL ist die Entwicklungsregel gewiss so zu formulieren, wenn man aber das ganze südostjakische Material des Wörterbuches durchsieht, muss unbedingt die Frage gestellt werden, ob sich nicht auch in der DN.-Mundart dieselbe Entwicklung abgespielt hat, oder ob man nicht mindestens einem Schwanken $o \sim o$ vor $i$ in DN. Rechnung tragen muss.

4.6. Es wurde schon einigemal darauf hingewiesen (s. oben S. $18,27,30$ ), dass im Südostjakischen neben $k$ keine velaren Vokale vorkommen ${ }^{1}$ (nur in einigen ganz jungen Lehn- oder Fremdwörtern, wie z. B. DN. toloknà $\cdot$, Kr. tōlâkna < толокно

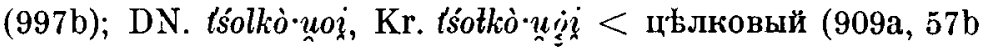
usw., aber s. OL 26 , vgl. OL 35,42 ). Neben $\chi$ sind hingegen die palatalen Vokale ziemlich selten, nach anlautendem $\%$ kommt ein diphthongiert ausgesprochener $e$-Laut $\left(<*_{i}\right)$ jedoch vor (s. oben S. 32). Da neben $k$ keine velaren Vokale anzutreffen sind, können vor $k$ die dort nicht vorhandenen velaren Vokale keineswegs palatalisierte Varianten aufweisen, sondern eben im Gegenteil, $k$ bzw. $\chi$ weisen in alten Wörtern eben auf die Palatalität bzw. Velarität der Wörter hin. ${ }^{2}$ Wieviel Jahre

1 Einigemal hat man den Eindruck, dass alle diakritischen Zeichen in der Feder geblieben sind, z.B. Kr. tukta 'anbeissen' (989b). Fil.,

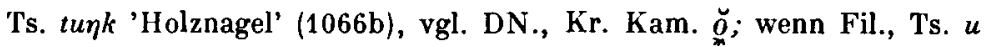
(und nicht $u$ ) richtig ist, müsste diesem $\eta k_{k}$ folgen!

a Vgl. Verf., NyK LXI, 251. Kansisto meint (FUF XIV, 74), dass im Wogulischen die Suffixe KU. $-k \ddot{a} t$ - (nach palatalen Verbalstämmen) $\sim-\chi \overline{\ddot{a} t}$ - (nach velaren) in ihrem Konsonantenelement auf die verschwundéne Vokalharmonie hindeuten. 
oder Jahrhunderte hier das Wort malt» bedeuten soll, erfordert noch eine weitere Untersuchung der jüngeren Lehnwörter, da in diesen $k$ in velaren und $\chi$ in palatalen Wörtern manchmal schon erscheint, und dadurch die Spaltung dieser Laute in zwei selbständige Phoneme, die in den Ostdialekten auch heute noch Varianten sind $(k, k)^{1}$, zustande kommen konnte, vgl. das anlautende $k$ - : $h$-im Ungarischen, Steinitz, GFgrVok. 22, Anm. 16; Verf., NyK LXII, 7-21 (ferner über den Zusammenhang zwischen Palatalität und $k$-Lauten Mikola, Népr. és nyelvtud. VIII, $23 \mathrm{ff}$.). Im Südostjakischen kann das Verhältnis $k: \chi$ um 1900 ungefähr so geartet gewesen sein, wie das Verhältnis $k$ - : $h$ - im Ungarischen vor der Landnahme war, als sich palatale mit $h$ anlautende und velare mit $k$ anlautende Fremdwörter bzw. Lehnwörter bereits in das Ungarische einzubürgern begonnen hatten.

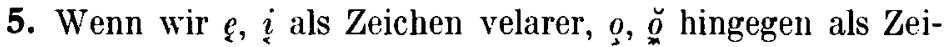
chen palataler Vokale betrachten, dann ist die Vokalharmonie in den Wörtern und in den Beispielsätzen des Wörterbuches im grossen und ganzen genau so geartet wie in den Texten. Der einzige Unterschied dürfte nur darin bestehen, dass in den Texten ein velares Wort entweder ein velares oder ein palatales Suffix bekommen kann - einmal etwa ein velares, ein anderes Mal ein palatales -, im Wörterbuch aber die beiden Möglichkeiten als fakultative Varianten angegeben werden können, was bei den Infinitivformen der Zeitwörter sofort in den Stichwörtern auffällt und gegen die Existenz der Vokalharmonie zu zeugen scheint. Ich hatte in Helsinki die Gelegenheit, KarJALAINENS Originalaufzeichnungen zu studieren. Da musste ich bemerken, dass er weder in der DN.-Mundart, noch in der $\mathrm{Kr}$.Mundart einen einzigen Infinitiv mit zwei verschiedenen Endungen aufgezeichnet hat. Ich habe bei beiden Mundarten Stichproben gemacht und die Originalaufzeichnung mit dem betreffenden Artikel des Wörterbuches verglichen. So konnte ich feststellen, dass im Wörterbuch das Wort entweder in der Form zu finden ist, wie es von Narygin an der Demjanka bzw. von Ivan

1 Oder ist auch hier die Spaltung, durch Eindringen der neuesten russischen Lehnwörter, eben im Entstehen begriffen (Verf., NyK LXI, 251)? 
Petrov und Vasilij Grigorjev in Krasmojarsk aufgezeichnet worden ist, oder wie es von Tailakov bzw. Matvej Kukyšev "verbessert» wurde; die Originalaufzeichnung wurde mit Bleistift geschrieben, die "Verbesserungen" wurden mit Tinte vorgenommen. Ab und zu wird die "Verbesserung" als Formvariante angegeben, aber hingewiesen wird nur dann darauf, dass die Gewährsleute, die die Korrekturen rorgeschlagen haben, eine andere Lokalmundart sprachen (DT. ist doch keine ober-, sondern eine unterdemjanische Mundart, und $\mathrm{Kr}$. ist auch nicht mit Kam. identisch), wenn durch die Korrektur eine A bweichung in der ersten Silbe entstanden ist. Genaueres hierüber könnte man nur damı aussagen, wenn man dis ganze Material der Originalaufzeichnung genau verzetteln "ürde. "Genau" soll hier die gewissenhafte und folgerichtige Angabe der Umänderung des korrigierenden Sprachmeisters bedeuten. Viele Wörter kommen in der Originalaufzeichnung öfters vor, und diese müssten nach einer solchen Verzettelung zusammengebracht und miteinander verglichen werden. Solange dies nicht geschieht, kann ich nur darauf hinweisen, dass es im Wörterbuch auch Formen gibt, die Übergangsformen zwischen der Originalaufzeichnung und der "verbesserten" Form darzustellen scheinen (aber etwal nur nach dem Wortartikel, mit welchem ich sie verorleichen konnte, woanders kann es vielleicht genau solche geben wie die Angabe, welche mir bei der stichprobenartigen Kontrolle im Wörterbuch als "Übergangsform» erschien).

Ich glaube, die Texte, die immer nur von einer Person aufgezeichnet wurden, spiegeln sicherlich einen existierenden Zustand wider, bei dem Wörterbuch muss aber unbedingt kontrolliert werden, ob das eine oder andere nicht aus zwei einander nahestrhenden Lokalmundarten kontrahiert worden ist.

6. Wenn wir die Regeln der südostjakischen Vokalharmonie so feststellen, dass den palatalen Wörtern palatale Suffixe angehängt werden, den velaren velare, bis auf Wörter mit inlautendem $i, t, n$ usw. oder einem $\underset{i}{i}(>i)$, oder einem hiatusfüllenden $i$-Laut, dann ist der Keim des Verschwindens der Vokalharmonie vermutlich schon in dieser Regel selbst zu finden. An oft gebrauchte velare Zeitwörter mit einem die 
palatale Fortsetzung erfordernden Inlaut wie z. B. lặid 'haben', zặida 'zurücklassen' usw. können verschiedene Ableitungrssuf-

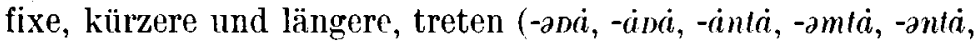

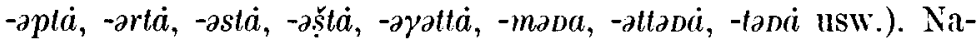
türlich sind bei diesen Zeitwörtern nicht nur die Infinitivzeichen, sondern alle Personalsuffixe palatal. Von diesen Zeitwörtern können damn die palatalen Suffixe analog auf velare Zeitwörter übertragen werden, da ja die Zahl der palatalen Wörter mit palatalen Suffixen, ja sogar ohne die velar brginnenden Wörter $2 / 3$ des Wortschatzes ausmacht (s. oben S. 4. Tabelle VI), wo eigentlich nur velare suffixe stehen könnten, wo kein inlautender Laut die palatale Fortsetzung beansprucht; so können die velaren Suffixe sogar langsam verdrängt werden, z. B. DN. tṣ̆ó $\chi \chi_{\text {ta }}$ 'schneien' (936b). Wenn es tatsächlich auch so eine Regel gibt, dass von der fünften Silbe an in Suffixen nur palatale Vokale stehen können (bis auf -ot, s. oben S. 4, Tabelle VI), dann begünstigt auch diese Regel die Verallgemeinerung der palatalen Suffixe und die Verdrängung der velaren. ${ }^{1}$

In der Ts.-, Sav.- und Kr.-Nundart ist in den Texten ziemlich häufig in der dritten, noch ofter in der vierten oder in der fünften Silbe statt -ən(-), -ân(-) eine "neutrale" Silbe -n̊n(-) zu finden; dadurch verschwinden genauso die letzten velaren Silben, wie bei den auf -3 auslautenden Wörtern, wo KARJALAINEN selbst bemerkt: "Streng genommen hätte man zwischen einer hinteren und einer vorderen Variante zu unterscheiden" (OL X, KT XXVI). Bei velaren Wörtern kommt sogar die Aufzeichnungsweise -ñn- vor, sie dürfte wohl als eine velare Variante gelten. ${ }^{2}$

Fremdwörter und Lehnwörter russischen Ursprungs komnten hauptsächlich in den dem russischen Einfluss stark ausgesetzten Süddialekten die Verbreitung der velar beginnenden und von den mouillierten Lauten ab palatal fortgesetzten Wörter för-

1 Interessanterweise sind auch im Wogulischen in den Mundarten ohne Vokalharmonie überwiegend die palatalen Suffixe bewahrt worden, s. z. B. in P. (KANnisto, FUF XIV, 72-3).

2 Nach Kannisto (FUF XIV, 43) verhalten sich die reduzierten Vokale in den Tavda-Dialekten des Wogulischen in der nichterstem Silbe als "neutrale» Vokale, in der ersten Silbe als palatale. 
dern, und da gibt es auch einen circulus vitiosus, das Vorhandensein dieser Wörter mit palatalisiertem, palatale Fortsetzung beanspruchendem Inlaut konnte das Eindringen der russischen Fremd- und Lehnwörter erleichtern. Ferner konnten die aus Postpositionen durch Agglutination entstehenden Kasusendungen zu einer Zeit, als das Kasussuffix noch in statu nascendi war (s. den Abl., S. 2), die Zahl der Wörter ohne Vokalharmonie vermehren und dadurch die uralten strengen Regeln der Vokalharmonie ein bisschen lockern.

Es ist leicht möglich, dass es zu der Zeit, als Streinitz das Südostjakische hören konnte, tatsächlich keine Vokalharmonie mehr in den ron ihm gehörten Mundarten gegeben hat. Wenn in den 35 Jahren, die zwischen den Forschungsreisen von KARJALAINEN und STEINITz vergingen, aus den lautlich konservativen V.-Vj.-Mundarten die,,$\ddot{\partial}$-Laute tatsächlich verschwinden konnten, konnte auch in den nichtkonservativen Südmundarten die den Keim des Verblassens auch sonst in sich tragende Vokalharmonie verschwinden.

Ich glaube, PATkaxov hat kein genügend geschultes Oh. gehabt, $1 \mathrm{~m}$ die Vokalharmonie entdecken zu können. KarJALainex und Paasonen hegten den Verdacht, dass es eine gegeben hat, da sie aber ihr Material nicht selbst bearbeiten konnten, sind sie nicht so weit gekommen, um die genaueren, vom Finnischen und vom Ungarischen abweichenden Regeln entdecken zu können, und STEINITz geriet schon allzu spät unter Südostjaken, um sie zu hören; da er sie im Ostostjakischen noch hö re n konnte, konnte er sie in KarJalainens Aufzeichnungen in den Ostmundarten auch s e h e n, aber im Süden, wo er sie nicht $\mathrm{g}$ e hö $\mathrm{rt}$ hat, konnte er sie durch Sehe $\mathrm{n}$ in KarJalainens Aufzeichnungen nicht entdecken;

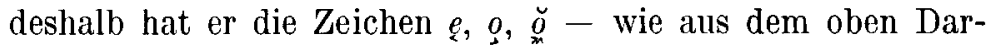
gelegten ersichtlich - irrtümlich als Varianten betrachtet. Da er in seiner Theorie ganz sicher war, hat er seine aus OL gezogenen Schlüsse anhand des viel reicheren Materials des Wörterbuches (wo auch suffigierte Wortformen zu finden sind) nicht kontrolliert und revidiert.

Ich wollte hier nur die Frage des Südostjakischen deskriptiv behandeln, auf die erschlossenen urostjakischen Laute habe ich 
nur gelegentlich hingedeutet, um zu zeigen, dass meine Auffassung, die in vielem mit der KaRJaLAINexs übereinstimmt, auch sprachgeschichtlich untermauert werden kann. Ich möchte in zwei Beziehungen jedoch auf die Wichtigkeit dieser Tatsachen für den finnisch-ugrischen Vokalismus hinweisen: Wenn die südostjakischen Verhältnisse hier richtig ausgewertet worden sind, dann folgt daraus, dass man bei der Untersuchung des Vokalismus nicht nur die unmittelbare Umgebung betrachten muss, sondern i m m e r d a s g a n z e W o r t, wie E. ItKonen es bei seinen Untersuchungen immer tut. Ferner unterstützen die südostjakischen Verhältnisse die Behauptung, dass die ostostjakischen Mundarten in ihrem Vokalismus tatsächlich archaisch sind und dem Urostjakischen sicherlich noch nahe stehen, wie KarJaLAINEN und Wichmaxw bemerkten und Steinitz es expressis verbis ausgesprochen hat (GFgrVok. 5, 63 ff., GOstjVok. 48 ff.). 
Tabelle I

Deklination

\begin{tabular}{|c|c|c|}
\hline & der Palatalen & der Velaren \\
\hline & $\begin{array}{lll}\text { DN. } & & \\
& & \\
& \text { Ts. } & \\
& \text { Sav. } & \\
& & \mathrm{Kr} .\end{array}$ & $\begin{array}{lll}\text { DN. } & & \\
& \text { Ts. } & \\
& \text { Silv. } \\
& & \text { Kr. } \\
& & \end{array}$ \\
\hline 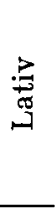 & 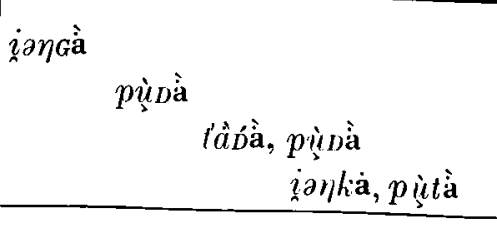 & 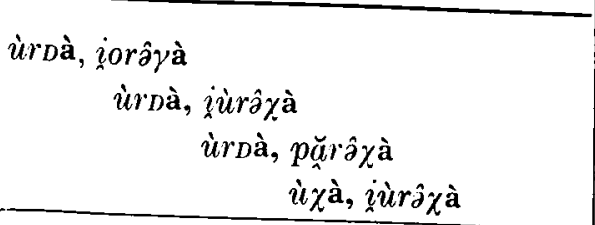 \\
\hline & 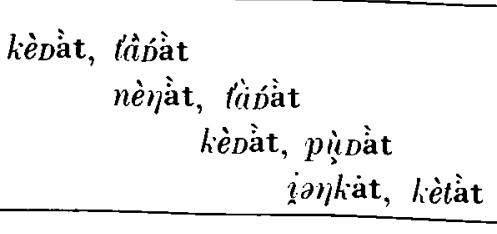 & 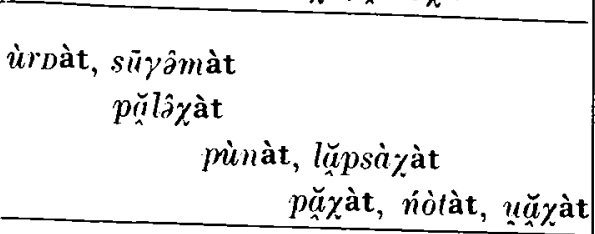 \\
\hline & 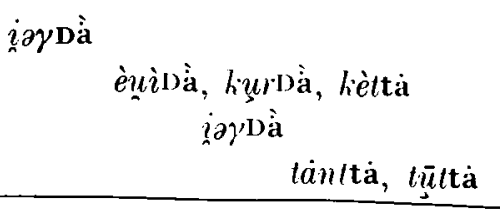 & 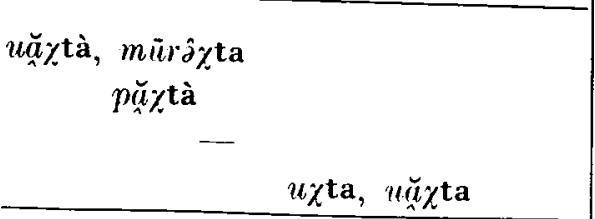 \\
\hline & 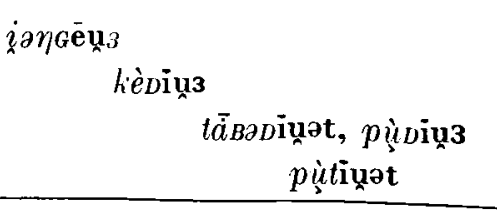 & 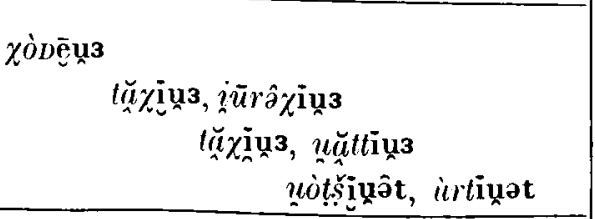 \\
\hline$\stackrel{\widetilde{\Phi}}{\stackrel{乛}{\Xi}}$ & 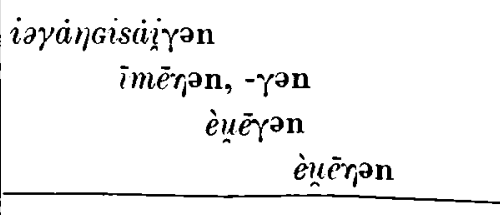 & 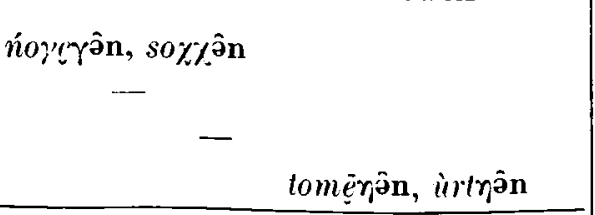 \\
\hline $\begin{array}{l}\vec{\Phi} \\
\stackrel{\Xi}{\Xi}\end{array}$ & 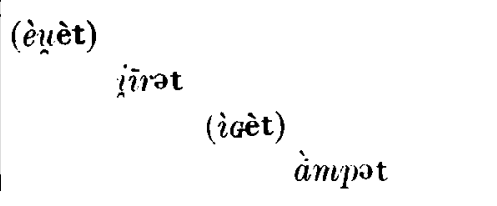 & 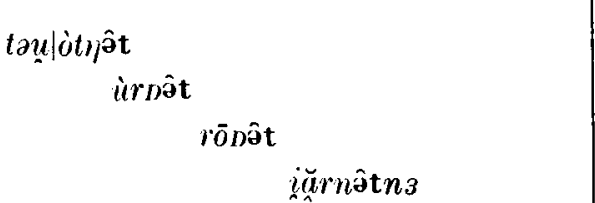 \\
\hline
\end{tabular}


'Tabelle II

Possessivsuffigierung

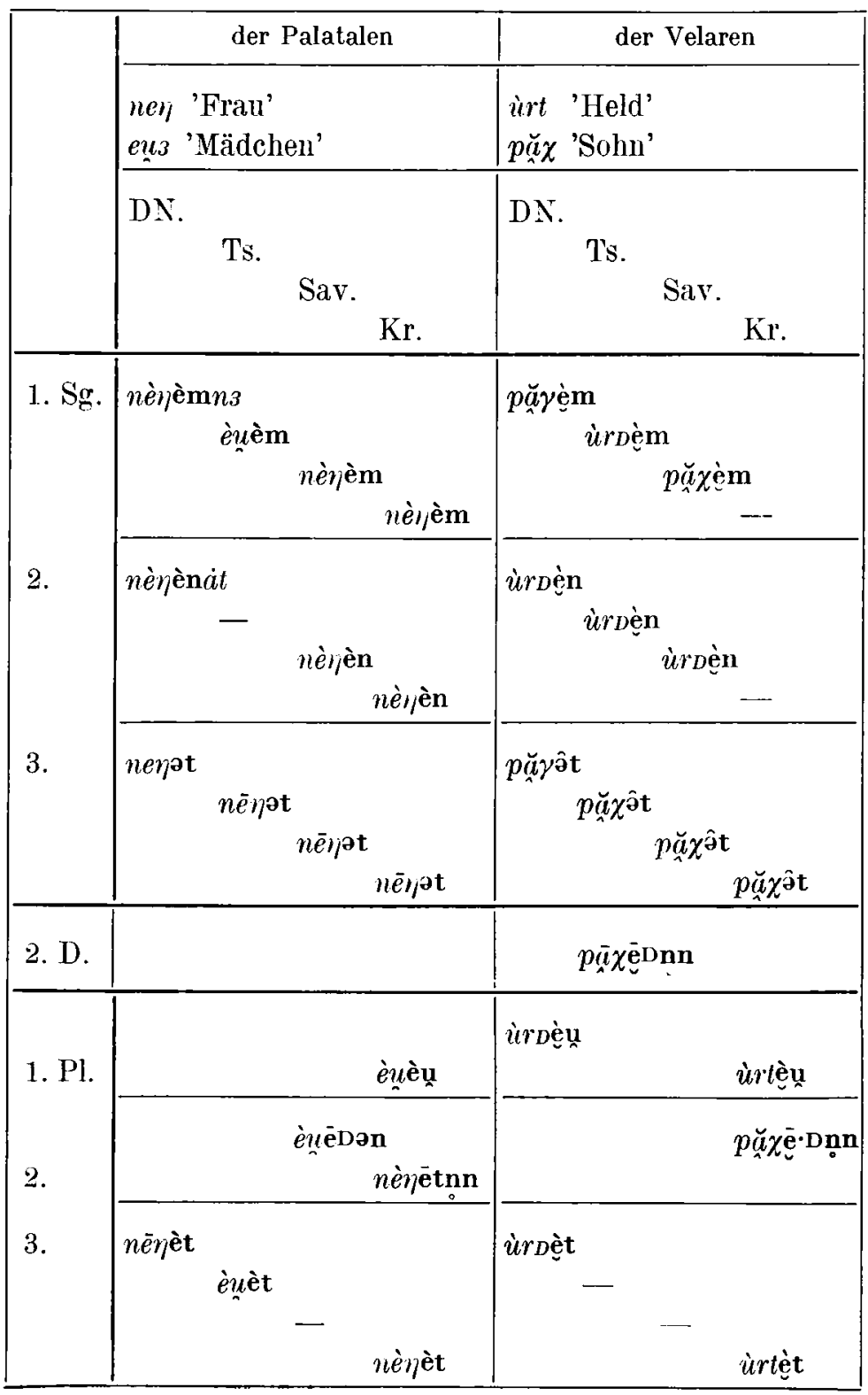


Tabelle III

Konjugation

\begin{tabular}{|c|c|c|c|}
\hline & der Palatalen & der Velaren \\
\hline & & 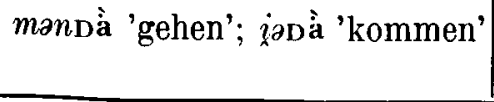 & $\begin{array}{l}\text { iăxtà 'gehen'; tặta 'kriechen'; } \\
\text { uttà 'leben' }\end{array}$ \\
\hline 总 & $\begin{array}{l}\text { 1. Sg. } \\
\text { 2. } \\
\text { 1. D. } \\
\text { 2. } \\
\text { 3. } \\
\text { 1. PI. } \\
\text { 2. } \\
3 .\end{array}$ & 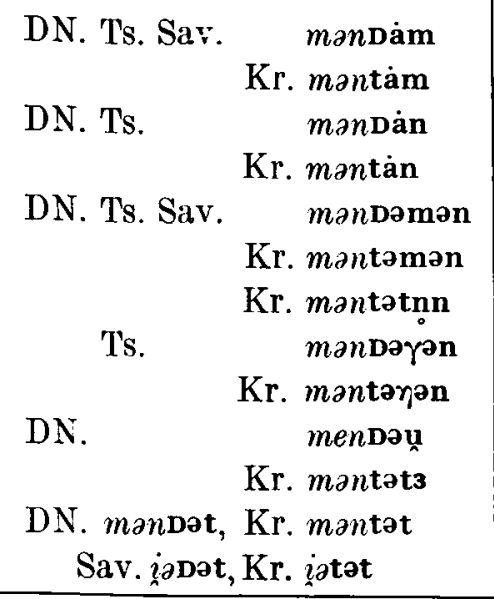 & 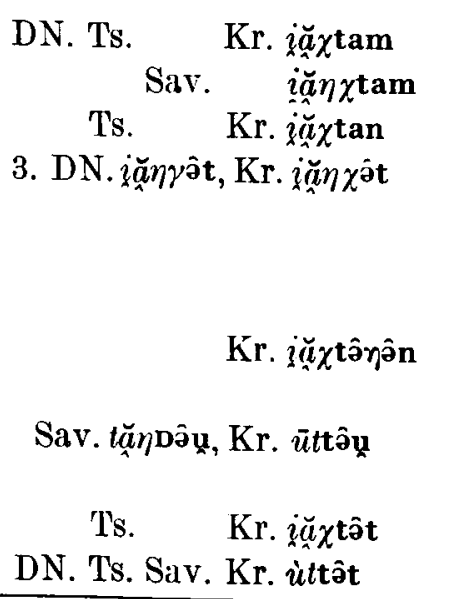 \\
\hline 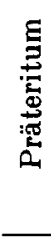 & $\begin{array}{l}1 .{ }^{1} \mathrm{Sg} \text {. } \\
\text { 2. } \\
\text { 3. D. } \\
\text { 3. Pl. }\end{array}$ & 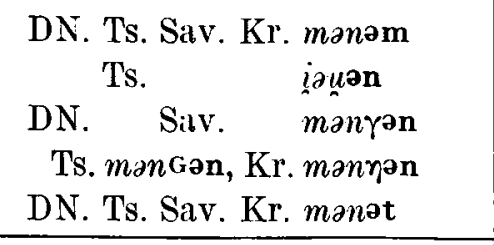 & 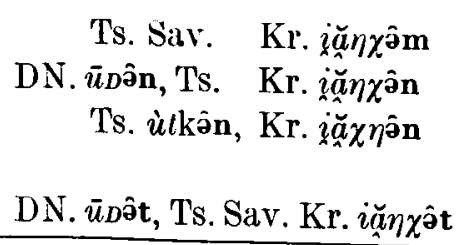 \\
\hline 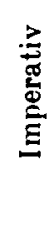 & $\begin{array}{l}\text { 2. } \mathrm{Sg} \text {. } \\
\text { 2. } \mathrm{Pl} \text {. }\end{array}$ & 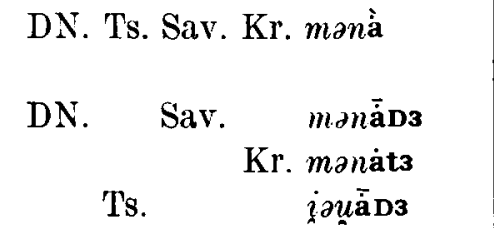 & 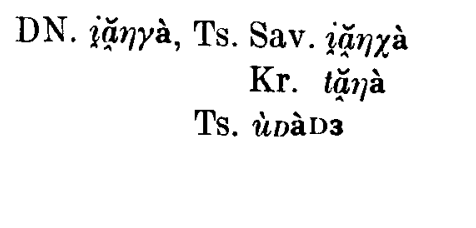 \\
\hline
\end{tabular}

I Oder Participium perfectum. 
Tabelle IV

Ableitungssuffixe

\begin{tabular}{|c|c|}
\hline der Palatalen & der Velaren \\
\hline 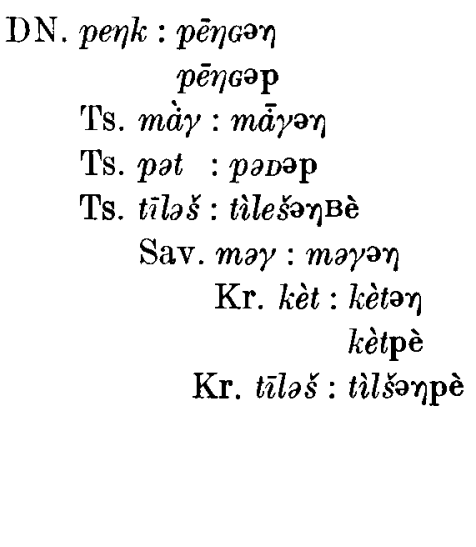 & 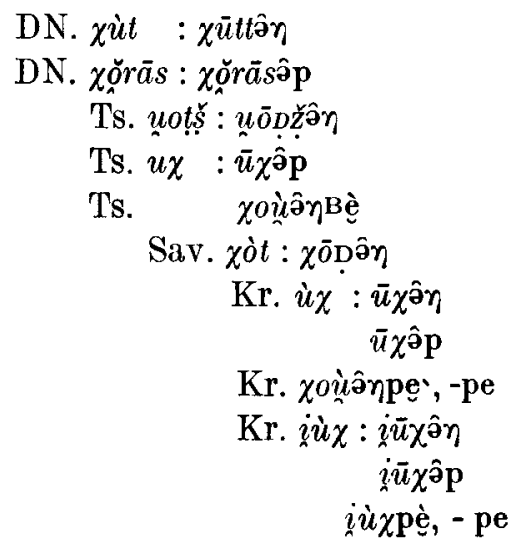 \\
\hline
\end{tabular}


Tabelle V

Deklination, Konjugation und Ableitungen velarer Wörter mit inlautendem $i, \dot{n}, t$ usw.

\begin{tabular}{|c|c|c|c|c|}
\hline & DN. & Ts. & Sav. & Kr. \\
\hline Lat. & 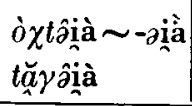 & 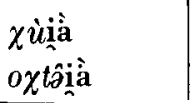 & $\begin{array}{l}\chi u \dot{i} \hat{a} \\
o \chi t a ̂ i a ́ a\end{array}$ & 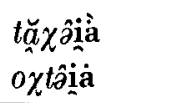 \\
\hline Instr. & $\begin{array}{l}\text { zòiàt } \\
\text { pùssàiààt }\end{array}$ & $\chi u \grave{i a ̀ t}$ & & $\begin{array}{l}\text { tuntâiàt } \\
\text { tăpâîàit }\end{array}$ \\
\hline Abess. & & 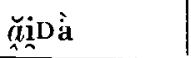 & & $\chi u t^{\prime} \mathbf{t a}$ \\
\hline $\begin{array}{l}\text { D. } \\
\text { Pl. }\end{array}$ & 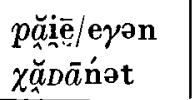 & $\chi u \mathbf{i}$ igon & $\chi u \mathbf{i} \gamma \partial \mathbf{n}$ & $\chi u$ injon \\
\hline $\begin{array}{l}\text { 1. Sg. } \\
2 . \\
3 . \\
1 . \mathrm{D} . \\
3 . \\
1 . \mathrm{Pl} . \\
3 .\end{array}$ & 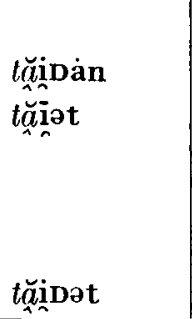 & $\begin{array}{l}\text { tặidàm } \\
\text { tặìàn }\end{array}$ & 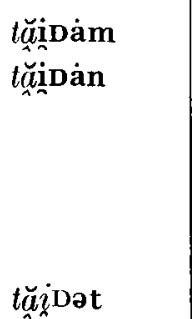 & 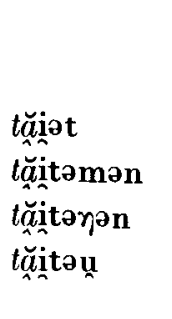 \\
\hline $\begin{array}{l}\text { 1. }{ }^{1} \mathrm{Sg} . \\
3 . \mathrm{D} .\end{array}$ & tặìm & 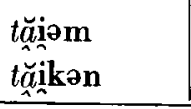 & tăরiam & tăiam \\
\hline $\begin{array}{l}\text { Impe- } \\
\text { rativ }\end{array}$ & & 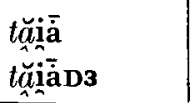 & & tặ̆iè \\
\hline $\begin{array}{l}\text { Ablei- } \\
\text { tungen }\end{array}$ & 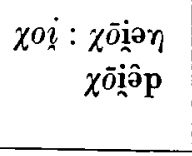 & 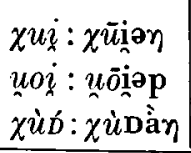 & 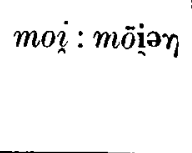 & 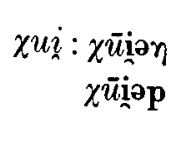 \\
\hline
\end{tabular}

1 Oder Participium perfectum. 


\section{'Tabelle VI}

Verhältnis der palatalen, velaren und vokalharmonielosen Wörter

\begin{tabular}{|c|c|c|c|c|c|c|c|c|c|c|c|c|c|c|c|c|}
\hline \multirow{2}{*}{ 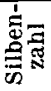 } & \multicolumn{4}{|c|}{ palatale } & \multicolumn{4}{|c|}{ velare } & \multicolumn{4}{|c|}{ vokalharmonielose } & \multicolumn{4}{|c|}{ insgesamt } \\
\hline & DN. & Ts. & av. & Kr. & & Ts. & av. & $\mathbf{K r}$. & DN. & Ts. & Sav. & $\mathrm{Kr}$. & DN. & Ts. & Sav. & Kr. \\
\hline 1 & 21,4 & 19,3 & 21,4 & 19,9 & 12,2 & $9,-$ & 12,4 & 11,3 & & & & & 33,6 & 28,3 & 33 & 31,2 \\
\hline 2 & 25,4 & 28,6 & 25,9 & 27,8 & $13,-$ & 18,3 & 16,1 & 16,3 & 6,6 & 7,3 & 5,7 & 4,3 & $45,-$ & 54,2 & 47,7 & 48,4 \\
\hline 3 & 8,7 & 8,6 & 9,6 & 9,1 & 3,9 & 4,1 & 3,5 & 4,1 & 4,1 & 2,7 & 3,7 & 3,1 & 16,7 & 15,4 & 16,8 & 16,3 \\
\hline 4 & 2,1 & 1,3 & 0,7 & 1,9 & 0,2 & 0,3 & 0,1 & 0,6 & 1,6 & 0,5 & 0,7 & 1,3 & 3 & 2,1 & 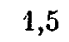 & 3,8 \\
\hline 5 & 0,35 & 0,1 & 0,2 & 0,2 & - & - & - & - & 0,35 & 0,1 & - & 0,05 & 0,7 & 0,2 & 0,2 & 0,25 \\
\hline 6 & 0,05 & - & - & - & - & - & - & - & - & - & - & 0,05 & 0,05 & - & - & 0,05 \\
\hline G. & $58,-$ & 57,9 & 57,8 & 58,9 & 29,3 & 31,7 & 32,1 & 32,3 & 12,00 & 10,0 & 10,1 & 8,8 & $\sigma, J$ & & $=0$ & 0, \\
\hline
\end{tabular}

Tabelle VII

Verhältnis der gleichlangen Palatalen/gleichlangen Velaren

\begin{tabular}{|c|c|l|l|l|}
\hline $\begin{array}{c}\text { Silben- } \\
\text { zahl }\end{array}$ & DN. & Ts. & Sav. & Kr. \\
\hline 1 & 1,75 & 2,15 & 1,73 & 1,76 \\
2 & 1,95 & 1,56 & 1,61 & 1,7 \\
3 & 2,22 & 2,1 & 2,74 & 2,22 \\
4 & 10,5 & 4,33 & 7, & 3,17 \\
\hline alle Palatalen & 1,98 & 1,83 & 1,8 & 1,82 \\
\hline alle Velaren & & & & \\
\hline
\end{tabular}




\section{Tabelle VIII}

Konsonantenumgebung von $e(e)$ in der ersten Silbe

\begin{tabular}{|c|c|c|c|c|c|c|c|c|}
\hline & \multicolumn{4}{|c|}{ DN. + DT. } & \multicolumn{2}{|c|}{ Irt. } & \multicolumn{2}{|c|}{$\begin{array}{c}\text { Kr. (Kam., } \\
\text { Ts.) }\end{array}$} \\
\hline & $-\gamma / \chi$ & $-k$ & $-\eta$ & $-r$ & $-\gamma / x$ & $-\eta$ & $-x$ & $-\eta$ \\
\hline$\underset{e}{e-}$ & $\oplus$ & + & 0 & & 0 & 0 & $\oplus$ & 0 \\
\hline$i e$ & + & & & & + & & t & \\
\hline ue- & + & & & 2 & & & $c$ & \\
\hline $\begin{array}{l}m e ̨- \\
p e-\end{array}$ & + & & & $i \uparrow$ & & & C & \\
\hline$r e-$ & + & & & & & & & \\
\hline$s e-$ & $\oplus$ & & & & & & + & \\
\hline tẹ- & + & & + & & 0 & & + & \\
\hline tẹ- & + & & & & $\oplus$ & & + & \\
\hline
\end{tabular}

Anhang. Belegstellen zu Tabelle VIII

(Die in OL $177-8$ nicht belegten Mehrsilber werden angegeben, um zu zeigen, dass die zweite Silbe immer velar ist.)

ę/Y-: DN., Fil., Kr. 11b; DN., Kr. 28a, 488a, DN. 27b, 188a, 266a, 438b, Ts. 220b | ęk̨-: DT. 31a | ęn-: DN., Koš., Kr., DN. plur. $e^{\vee} \eta \gamma_{\nless} \hat{t}, 36 \mathrm{a}, \mathrm{DT}$. 39a, DN., Kr. 39b, Ts. 40a.

ie $\chi / \gamma-:$ DN., Koš, Fil., Sogom, Tš., Kr. 146b, DN. 286b, 721a, 728a, 966a, Kr. 897a.

unę $/ \gamma$-: DT. 214a, 391a, Tš. $214 a$.

?męr-: D. męràièu (pl. l p. ps.) aber nur palatal: DN. mèràịa,

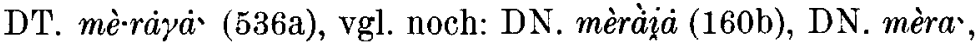
'Mass' (OL 157).

pę $/$ /-: DN. 668a, DN., Kr. 679b.

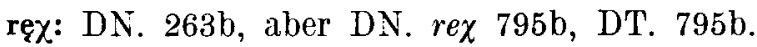

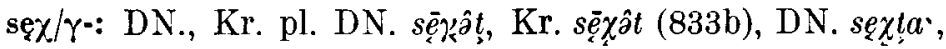

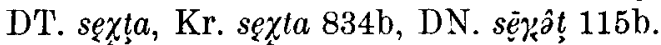

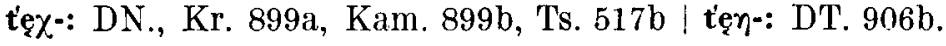
(DN. s. OL 177).

tę $\chi / \gamma$-: DN., Fil., Ts., Sogom, Kr. 985b, DN., Koš., Ts.,

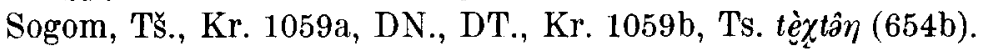


Belege zu den Tabellen IX-XI

Die Belege zu Tabelle IX - XII, XV sind nur selten Grundwörter, die meisten sind Ableitungen oder Wörter mit Kasusund Personalsuffixen. Da für unser Thema nur die phonetische Umgebung eine Bedeutung haben kann, sind die Belege alphabetisch nach dem vor $\varepsilon(e)$ bzw. $i(i)$ stehenden Konsonanten geordnet, d.h., wie sie der Reihe nach in die Tabellen hineinpassen. Wenn eine phonetische Umgebung in mehreren Beispielen vorkommt, werden mehrere zitiert, um gleichzeitig auch die Belastung zu illustrieren. Die Verbindungen -em, $-e ̨ n$, bzw. $-e \eta,-e p$ sind in Possessiv- bzw. in Ableitungssuffixen sehr häufig.

Im Stamm der Wörter ebenso wie vor Ableitungs-, Kasusund Possessivsuffixen ist es keine Seltenheit, dass $e(e)$ in der zweiten Silbe parallel in mehreren Mundarten erscheint. Ebendeshalb wird dadurch Raum erspart, wenn das Belegmaterial zu den Tabellen IX-XI zusammengefasst wird. Auf diese

Tabelle IX

Konsonantenumgebung von DN. $e$ in der zweiten Silbe*

\begin{tabular}{|c|c|c|c|c|c|c|c|c|c|c|c|c|c|c|}
\hline & $\cdot \emptyset$ & $-i$ & $-\underline{u}$ & $-k$ & $-\gamma$ & $=x$ & $-\eta$ & $-m$ & $-n$ & $-p$ & $-s$ & $-t$ & $-\dot{s}$ & $-t$ \\
\hline$i-$ & & & & & + & & + & & & & & & & \\
\hline$\underline{u}-$ & & & + & & & & + & & & & & & & + \\
\hline $\mid \gamma-$ & & & & & & & + & + & + & + & + & $\oplus$ & + & + \\
\hline $\mid x$ & & & & & + & & + & + & & + & & & & + \\
\hline$l-$ & + & & + & & & & + & + & & + & & + & & \\
\hline$m-$ & & & & & & & & + & + & & + & & & + \\
\hline$n-$ & + & & + & & & & & + & & & & & & \\
\hline $\begin{array}{l}n- \\
p-\end{array}$ & & + & & + & & & + & + & & & & + & & \\
\hline$r$ & + & & & & & & + & + & & + & + & + & & + \\
\hline $\begin{array}{l}s- \\
\check{s}-\end{array}$ & & & & & & & + & $\begin{array}{l}+ \\
+\end{array}$ & & & + & & & + \\
\hline ťs & + & & & & & & + & + & & & & & & + \\
\hline$\ddot{t}-$ & + & & + & + & + & + & + & + & + & + & + & + & & + \\
\hline
\end{tabular}

* Vgl. noch DT. -n ex/y-. 
T'abelle X

Konsonantenumgebung von Ts. $\ell(e)$ in der zweiten Silbe

\begin{tabular}{|c|c|c|c|c|c|c|c|c|c|}
\hline & $-\mathscr{O}$ & $-\underline{\mu}$ & $-k$ & $-\eta$ & $-m$ & $-n$ & $-p$ & $-t$ & $-t$ \\
\hline $\mid \gamma-$ & & & & 0 & $\oplus$ & & 0 & & \\
\hline $\mid x^{-}$ & & & & + & $\oplus$ & & + & & + \\
\hline$l-$ & & 0 & & & 0 & & & + & 0 \\
\hline$m-$ & & & & & 0 & & & & \\
\hline$n-$ & 0 & & & 0 & $\oplus$ & & & & \\
\hline$p-$ & + & & & & 0 & & & & \\
\hline$r$ & + & & & & 0 & & 0 & $\oplus$ & \\
\hline$\stackrel{t s}{s}-_{t^{-}}$ & & & $\begin{array}{l}+ \\
0\end{array}$ & & $\begin{array}{l}\oplus \\
\oplus\end{array}$ & $\oplus$ & 0 & & $\oplus$ \\
\hline
\end{tabular}

Tabelle XI

Konsonantenumgebung von $\mathrm{Kr} . \ell(e)$ in der zweiten Silbe*

\begin{tabular}{|c|c|c|c|c|c|c|c|c|c|c|}
\hline & $-\emptyset$ & $-u$ & $-k$ & $-\eta$ & $-m$ & $-n$ & $-p$ & $-s$ & $-t$ & $-t$ \\
\hline & & & & & & & & & & \\
$u-$ & & & & + & & & & & & \\
$\chi x-$ & + & & & + & + & + & & & + & \\
$l-$ & & & & + & & & & & + & \\
$m-$ & & & & & + & & & + & & \\
$n-$ & + & + & & 0 & + & + & & + & & \\
$\dot{n}-$ & & & & + & & & & + & + & \\
$p-$ & & & + & & & & & + & + & + \\
$r-$ & + & & & + & + & & & + & + & + \\
$s-$ & & & & & + & & & & & \\
$t-$ & & & & & + & & & & & \\
$t \underline{s}-$ & & & & & + & & & & & 0 \\
$t-$ & + & & + & + & + & + & + & + & & 0 \\
\hline
\end{tabular}

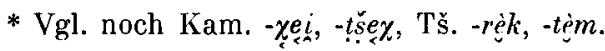

Weise ist es sogar möglich, nicht nur die drei am besten bekannten Mundarten (DN., Ts., Kr.) zu veranschaulichen (wie es tabellarisch geschah), sondern auch auf die übrigen, tabellarisch nicht dargestellten Mundarten hinzuweisen. Dem $e(e)$ der zweiten Silbe entspricht ab und $z u$ in anderen Sïdmund- 
arten ein $i(i)$, deshalb vgl. Belege zu 'Tabelle XV. Zu beachten: die erste Silbe ist immer velar (oft auch die dritte), s. jedoch $e \sim e$.

-ieץ: DN. păi păiè 'von Bodenfrost durchsetzt' (657a).

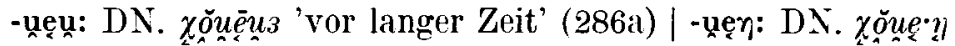

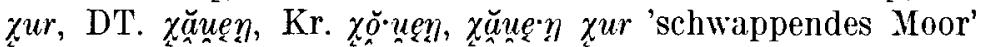

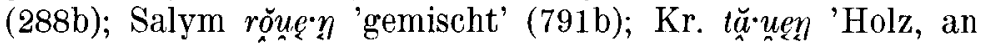
dem eine durch einen Ast entstandene Biegung auftritt' (1045b)

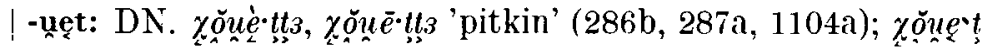
['Moorlachen'] (288a).

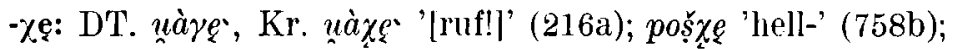

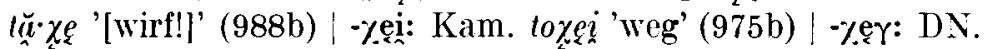

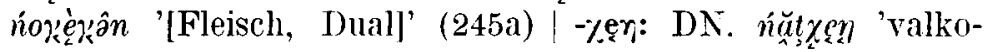

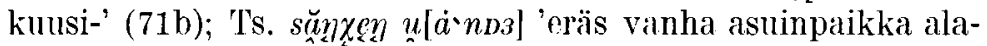
Kondalla' (229a); Kr. mo*๕!l, Kam. moxe!l 'Karauschen-'

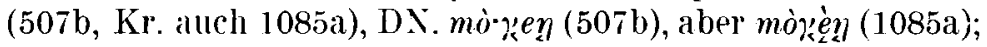

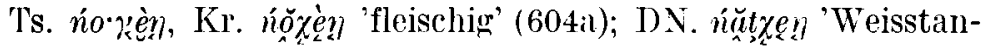

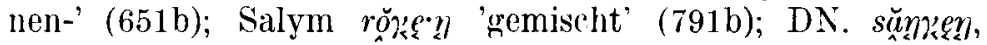
Kr. să ’nxen 'sandgemischt' (850b); Salym tăyjèn '[wogend]'

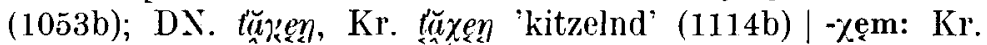

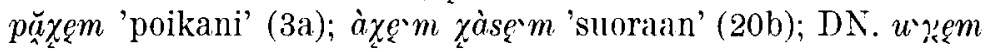

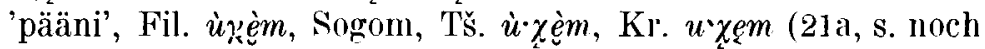
DN., Kr. 447a, 821b, DN. 409a, 512h, 523b, Ts. ù ұ̀̀m $821 \mathrm{~b}, \grave{e}$ 239a, Kr. 284a, 361 b, 383a); DN. u’ 832b); Kr. uӑұgm 'rahani' (209a, 1019b); 'Ts. ùą̀èm, Kr.

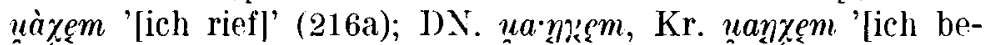
haue]' (220a); T's. lặmeẹmonad 'sich spalten' (479b); DN. mònęm 'lapseni' (503a); DN. măx $\bar{e} \cdot m a p \dot{a}$ 'sich losreissen' (511a), DN.

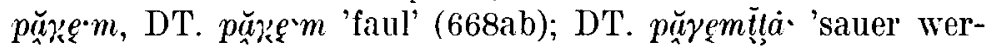
den' (669a); DN. poryem, Ts. pòrzèm, Kr. porzem 'vatsani' (726a, DN. 423b, 883a); Ts. rò 'રxèm, Kr. ròzem '[mein Kragen]'

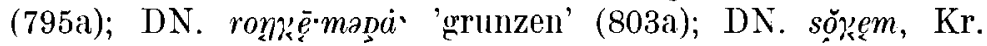

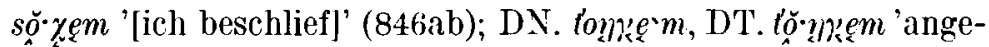

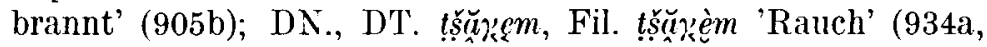

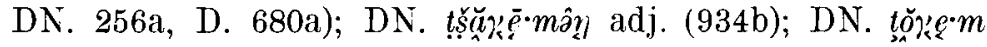

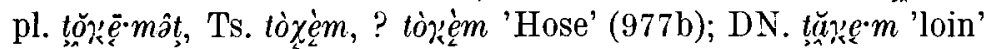

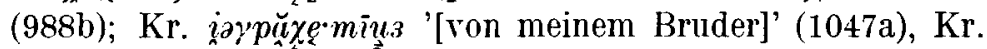




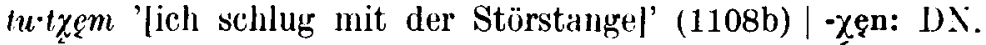

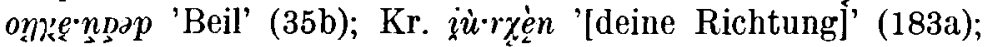

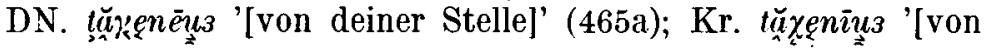
deiner Stelle]' (470a); DN. moxe ${ }^{-} n 3$ tor 'Karauschentümpel' (507b); Kr. jenatiù $\chi e \cdot n \dot{a}$ ' '[auf deinem Holz]' (717b); DN.

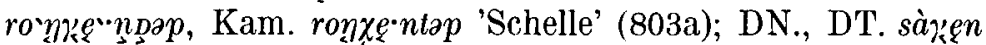

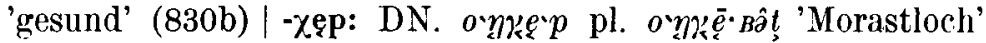

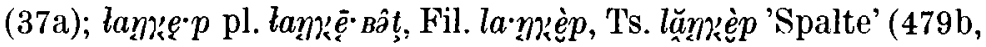

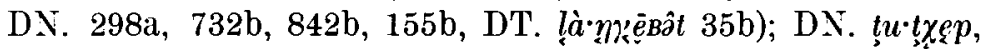

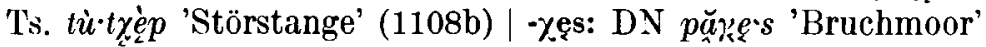

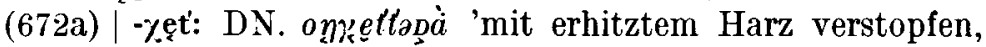

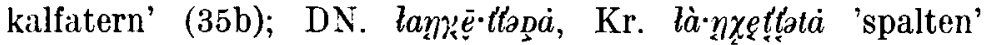

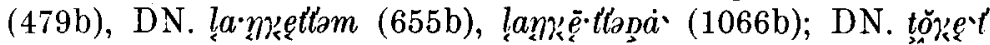

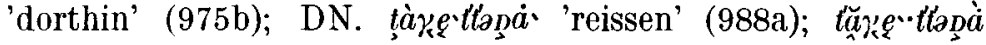

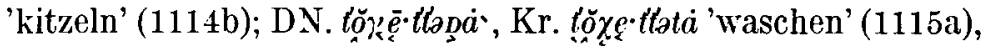

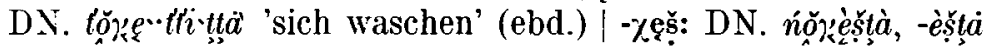

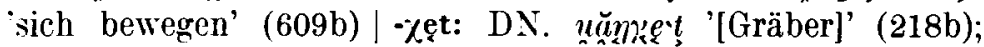

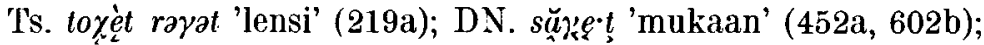
mòxet, '[Karauschen]' (507a); riă.txęt '[Weisstannen]' (651a);

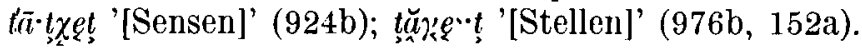

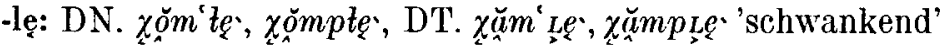

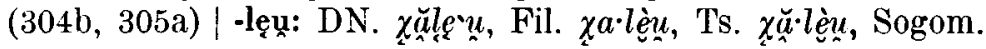

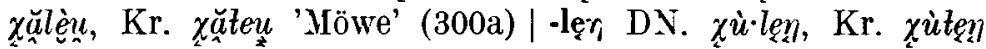
'schmutzig' (299b) | -lęm: DN. mă

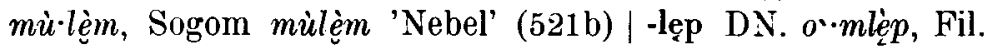

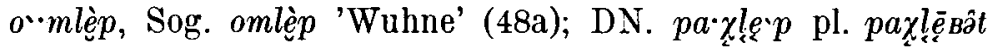

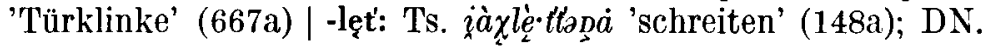

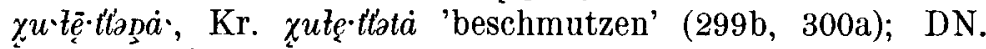

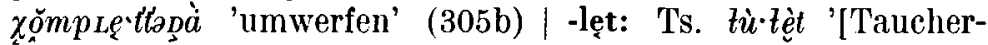
vögel]' (482a).

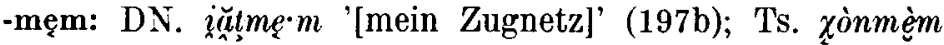
'[ich nahm es auf den Rücken]' (319a); Kr. nù męm '[ich erinnerte mich]' (581b); DN. sặ.rmęm 'surmani' (878b); șàmęm 'meine Kerze' (942b); Ts. tṣ̆oṣ̆mèm '[ich goss es aus]' (961b); Kr. tùrmęm '[mein Himmel]' (1015b)|-męn: DN. xִă.ţmęn 'kuollen' (373b); D. sărmęn 'kuolemasi' (878b)|-męs: Kr. ămęsta 'muhen' (44a); DN. to.mèstà 'lauern' (1071b, 371a)। -męt: D. ùtmèțuş 'so' (106a). 


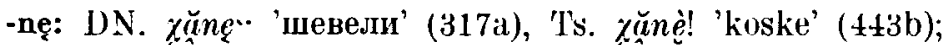

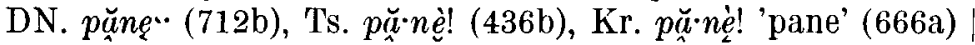

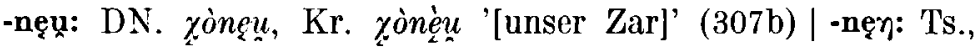

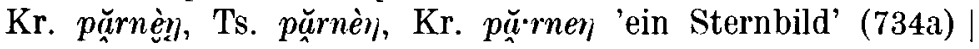
-nęm: DN. uònęm '[meine Schulter]' (226b); Ts., Sogom ұ̨onèm, Kr. xŏnęm '[mein Bauch]' (308b, Ts. ̀̀ 435b, 750b); Ts. pănèm '[ich legte es hin]' (712b)|-nęn: Kr. pănęn 'panet' (470a)| -nęs: Kr. jăănę'sta 'schwingen' (174a).

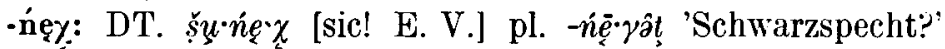

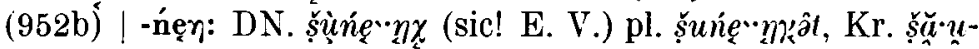

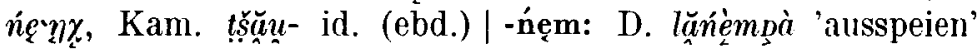
(486a).

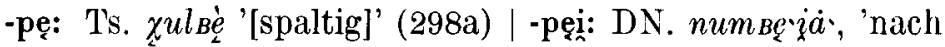

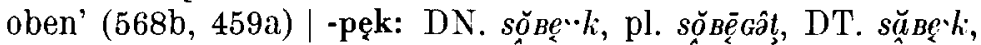
Kr. so.pe $e^{\cdot k}$ sope $\bar{e} \cdot k \hat{t} t$ (sic! E. V.) < сапокъ $(864 \mathrm{~b}, \mathrm{DN} .201 \mathrm{a}$, 242b, 293a, 1073b, Kr. 243a) |-pęm: Ts. untpèm 'meine Schwiegermutter' (60a) | -pęs: Kr. tşsùpèsta, -esta 'zerspalten' (954a)|

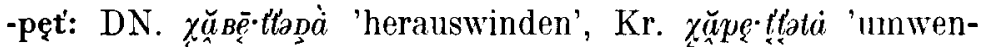
den' (326ab).

-rę: DN. iŏurę", DT., Koš. iŭurę, Fil. iơ urę, Ts. iŏ urè, 'schief' (137b); Kr. nărè̀ 'abschüssig' (591a); nooxyrę 'glätte'

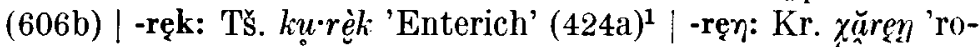

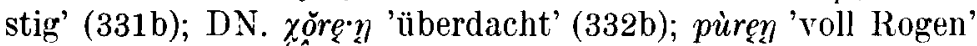

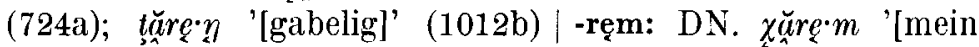

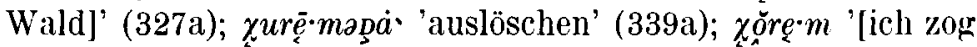
es ab]' (340b); morę $\cdot m a d \dot{a}$ ' 'zerbrechen' (536b); ńóxrç.m '[ich hobelte]' (606b); 'Ts. nonžèm '[ich nagte ab]' (614b); Kr., Kam. ńànem pòręm '[mein Brot als Wegzehrung]' (624b, 720a); DN. săx (921a); şoręm pl. şorẹ̄mât [0: şoręemât E. V.] 'Rinnsal' (958a, 160b); tòxrèm '[ich schloss zu]' (981b); tarę-mapă 'до Черемкойскихъ' (1031a); țăę̄-məpà 'schneiden' (1093b)|-ręp: DN.

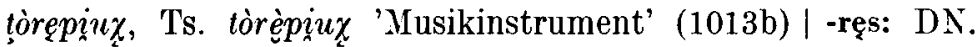

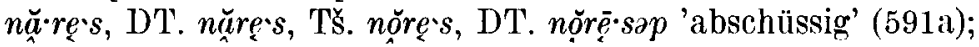

1 S. oben S. 9 über $u_{\circ}$; das Wort ist in den DN., Fil., Ts., Kr., Kam.Mundarten ganz palatal, mit $[\ddot{o}] \sim[\ddot{u}]$ in der ersten, mit $e$ in der zweiten Silbe; Sogom kurèk ist wahrscheinlich ein Druckfehler. 
DN. poręsța 'bewirten [die Götter]' (723b); Kr. lărèsta, -esta 'schnauben' (1025b)|-ręt': DN. ò rętt'apà, Kr. òręttota 'abtra-

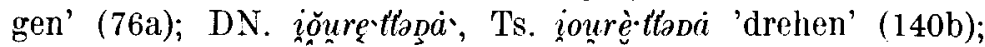
DN. mòrę"ttapà', Ts. mùrè

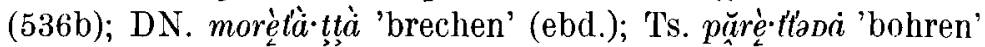

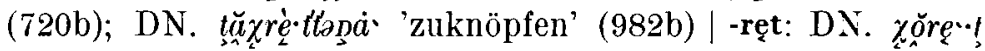
'Lockvögel' (331a); xŏrępà 'tuppeen' (332a); karanò̀ręt ' 'See-

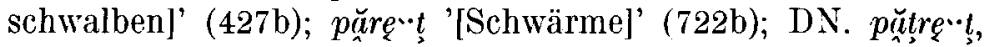
Kr. pătręt 'heidän keskustelunsa' (766b); DN. tòrę̨t '[Kraniche]' (1013a, 722b).

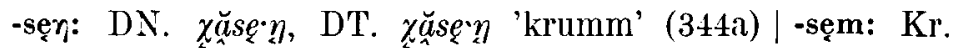

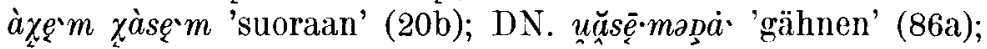

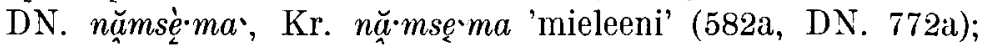
DN. pù-sęm 'ich melkte' (744b); toұsęm 'мой другъ' (1056b)| -sęs: DN. uăsę"sța 'gähnen' (86b); uă sęsitțà id. (ebd.)| -sęt: [? DN.] uăsę`t '[Ruten]' (250a); DN. zăàè̀! '[Biegungen]' (343b).

-t'ęm: Kr. soțt'em '[ich fing]' [sic! E. V.] (883b).

-šęm: DN. òšęm '[mein Schaf]' (97a).

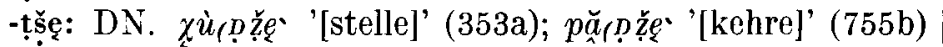

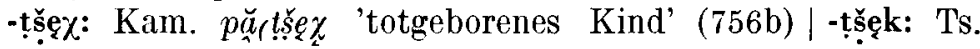

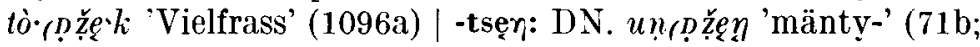

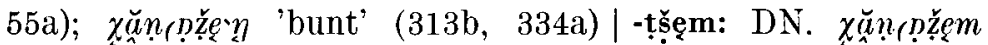

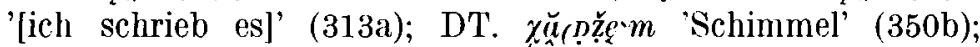

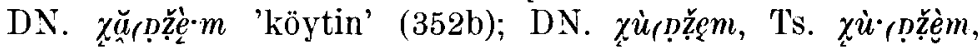

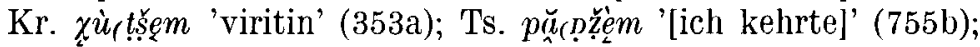

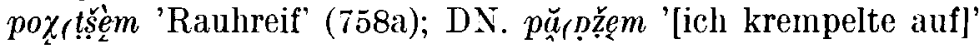

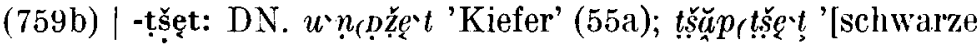
Johannisbeeren]' (957b).

-tę: DN. u“ntev '[lern!]' (62b); ńóxtę '[bewege!]' (67b, 1086b);

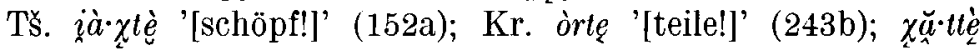

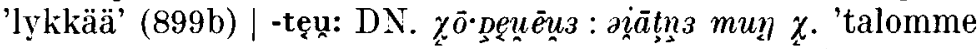

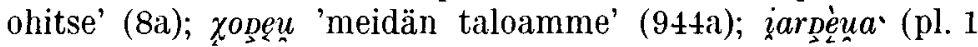

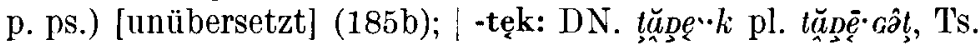

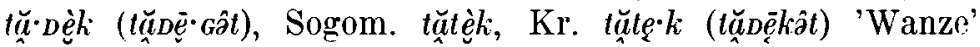

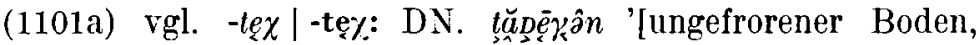

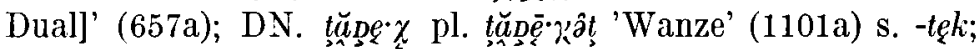

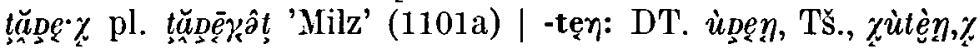




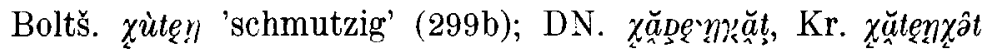

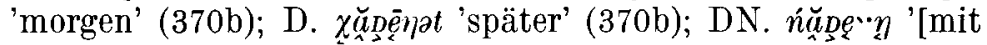
Jahresring versehen]' (642a); DN. ńắpen, Kr. náătèn '[schleimbedeckt]' (646b); Tš. rùtèn 'blatternarbig' (812a)|-tęm: Ts. nu.ptę'mnз 'in meinem Leben' (4b); DN. mán|ăttem '[von mir]' (8a, 650a), Kr. mä|ăttęm (650a); Ts. omvèm '[ich stellte hin]' (46b); DN. w•nittem 'ich lernte' (62b); Kr. òt tèt ătèm 'Gedächtnisfeier (nach meinem Tode)' (100b); DN. ottem '[ich fügte hinzul' (130b), Ts. ioxtèm '[ich kam]' (152b); DN. io ‘n npèm, Fil. ‘̇ò'ndèm '[ich nähte]' (175a); Tš. i̊o tè̀m '[ich flickte]' (199a); Kr. uaxtęm '[ich rufe]' (216a); DN. òrọèm, Ts. òroèm, Kr. ortęm '[ich teilte]' (243b); Ts. [uòro] èm '[ich schob]' (245a); DN. uònęm '[ich sehe, weiss]' (268a, 280b, 281a), Kr. ù.tèm (183a, 766b); DN. joxtèmnз '[von meinem Schiessbogen]' (284a,

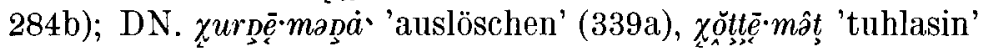

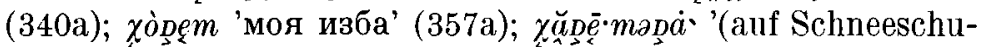

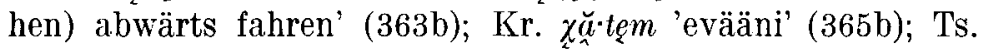

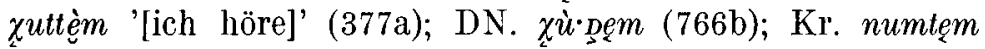
'muistan' (581b), DN. nùptem 'eläissäni' (585b); Ts. nóóxtèm '[ich rückte beiseite]' (610b); DN. noxtem '[ich jagte]' (611b); ńắpē-məsà 'schnell ablecken' (650b); Kam. paștem, -ęm (?) 'schwach'

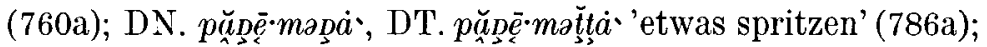
Kr. soxtęm ?' '[ich beschlafe]' (846b); DN. sùvèm '[mein Darm]' (888a); Kr. tùrmèm xăttèm ùtęm 'elin ikäni' (1015b); DN. tù'Dèm, Kr. tù.tèm 'ich bringe' (1031b); Ts. to ‘xtèm '[ich biss]' (1057b);

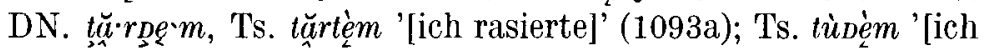
kaufte]' (1101b) |-tęn: Ts. oxtęns, Kr. oxtęeñ 'sinua vastaan' (21b); DN. o“De`n '[dein Jahr]' (109b, 496a); Sogom ùdèn 'du weisst es' (183a); Ts. ùrDèn '[dein Herr]' (546b); DN. šă

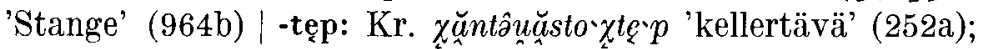

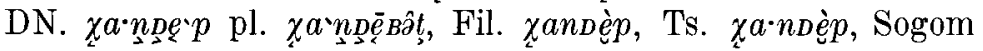

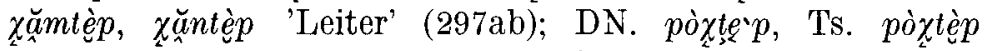
'Fischgabel' (677b)|-tęs: DT. uorpè sța' 'zu kommen befehlen' (84a); DN. $\chi \breve{a ̆ D} \dot{e} \cdot \operatorname{sta} \dot{a}$ 'auf Schneeschuhen, einem Rutscher

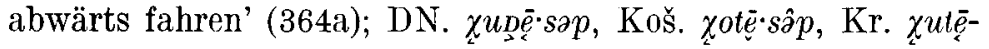

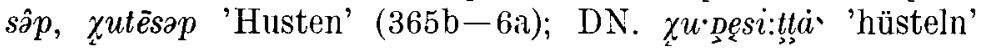

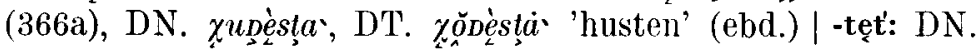

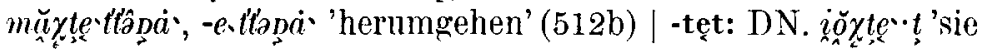




\section{Tabelle XII}

Konsonantenumgebung von $\rho(e)$ in der dritten Silbe

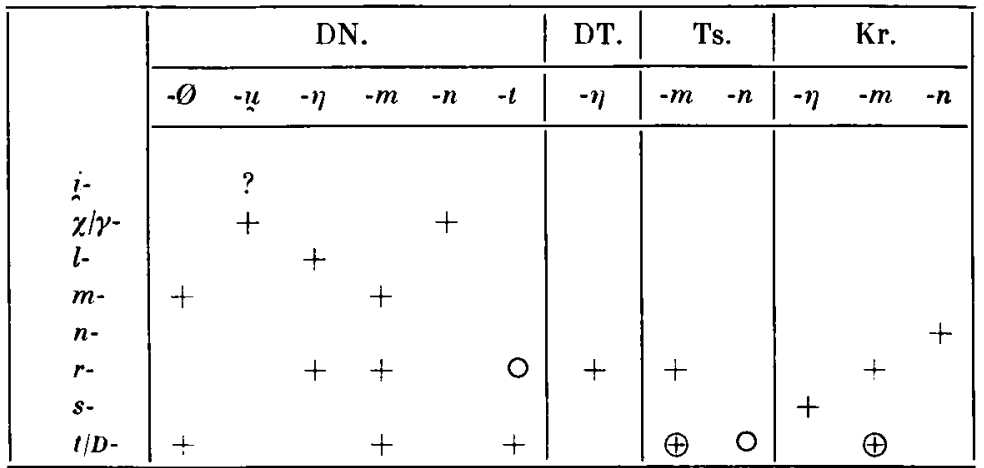

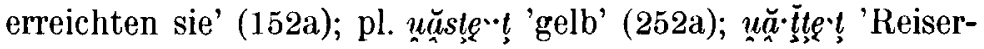

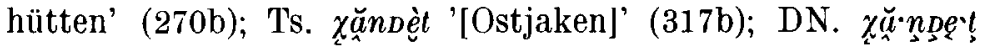

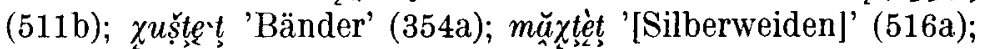

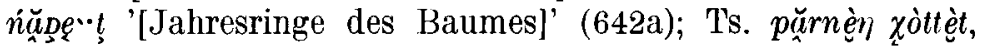
Kr. părnèn xòttèt, păarnen xo.ttet 'ein Sternbild' (734a); DN.

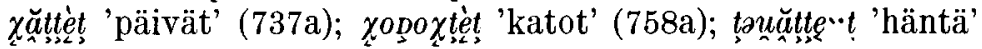
(778a, 1086b); sustęt', Ts. su'stèt '[Bandwürmer]' (881b-2a);

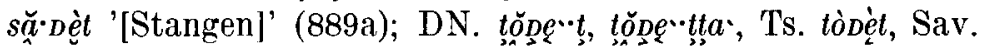
tòvèt 'von dort, von da' (976a); DN. tăptệ! ’'[Pfeifenten]' (1083b).

\section{Tabelle XIII}

Velares $e$ in Einsilbern in den Südmundarten

\begin{tabular}{|c|c|c|c|c|c|c|c|c|c|}
\hline DN. & DT. & Koš. & $\mathrm{Kr}$. & $\mathrm{DN}$ & $\mathbf{K r}$. & Ost & Bedeutung & OL. & $\mathrm{KT}$. \\
\hline$s e^{\prime} \chi$ & & & $\operatorname{se\chi }$ & & & V.-Vj. Trj. six & Ballen & 177 & $833 b$ \\
\hline$t e \cdot x$ & & & $t e \cdot \%$ & $t^{\prime} e \cdot y$ & $t e \cdot \gamma$ & Trj. ti. & dicht, fest & 178 & $899 a$ \\
\hline rę & $r e ̨ \%$ & & & rèy. & & & Schmutz & $\cdots$ & $795 b$ \\
\hline$\because n \eta$ & & ètuz & $e \eta \chi^{1}$ & - & - & V.Vj. $i \eta_{k}^{k}$ & Rebhuhn & - & $36 \mathrm{a}$ \\
\hline
\end{tabular}

${ }^{1} \mathrm{KO}$ ist vermutlich ein Druckfehler statt $\mathrm{Kr}$. 


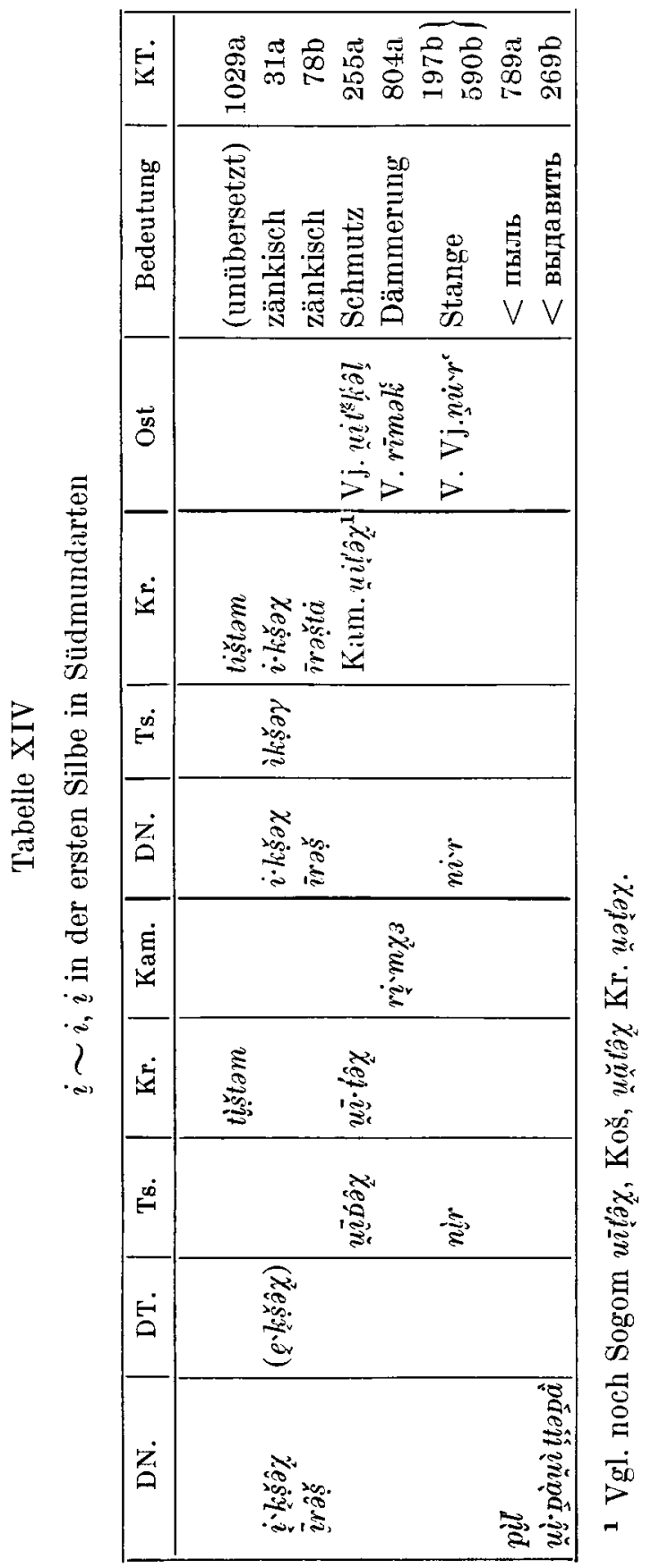




\section{Belege zu Tabelle XII}

-ięu: D. mèràièu (pl. l p. ps.) (536a), s. jedoch S. 46.

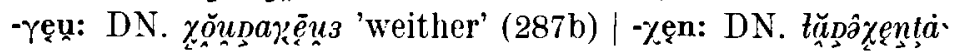

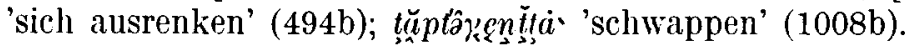

-łęn: DN. pặałę̣ 'Steck-' (766a).

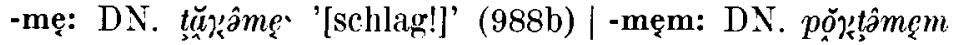
'[meine Wange]' (675a). || -nęn: Kr. sàràn[èn] 'kattila(si)' (872a).

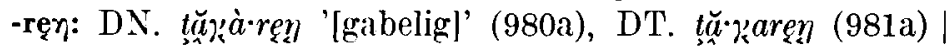

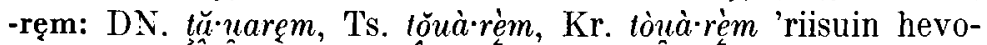
sen' (973a)|-ręt: DN. pl sọ̆) à̀-rèț 'Zwieback' (839b).

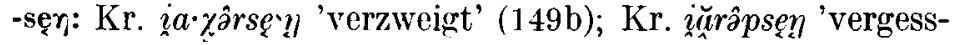
lich' (185b).

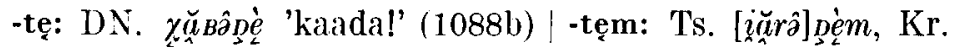
iŭrâtęm '[ich vergass]', Kr. iŭrâttęm '[ich vergesse]' (185b); Ts. iŭuâà̀m 'ich vergass' (1023b) Kr. xürâttèm '[ich lösche aus]', xüuâtèm (pret.) (339a); Ts. lünxânèm '[ich spaltete]' (479b);

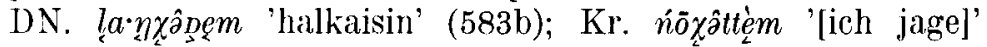
(611b); Ts. nórâpèm '[ich drückte es nieder]' (635b); DN. pakț-

\section{Tabelle XV'}

Konsonantenumgebung von $i(i)$ in der zweiten Silbe

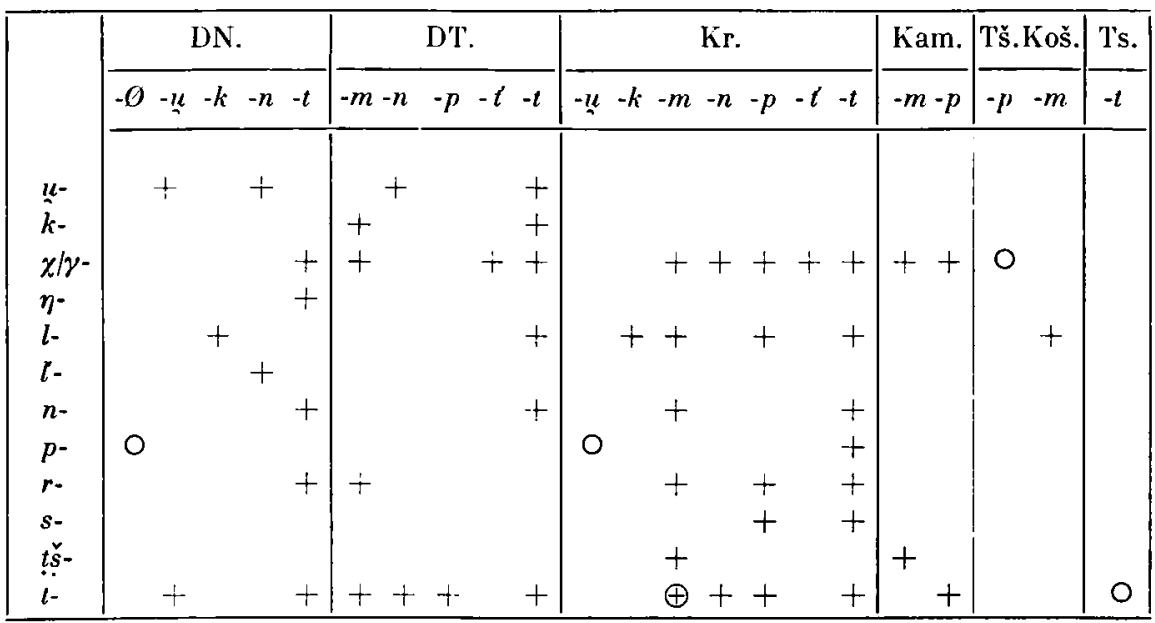

$1 \mathrm{Vgl}$. .. 47-54; Relege «u den Tabellen IX-XI. 
ămà̀ Dęem '[meine Fähigkeit zum Dulden]' (681b) | -Dęn: Ts.

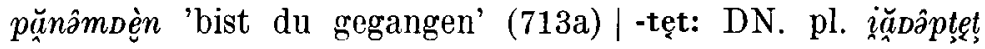
'Flicklappen' (199a); D. ặmàDèt 'fähig' (800b).

\section{Belege zu Tabelle XV}

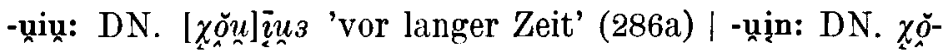

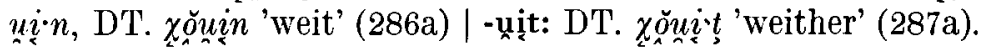

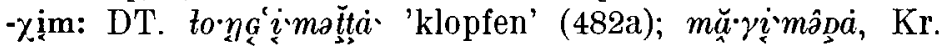

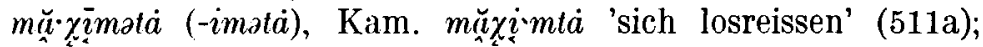
Kr. tanximata 'sich spalten' (479b); tonxiz 'angebrannt' (905b);

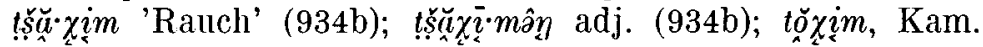

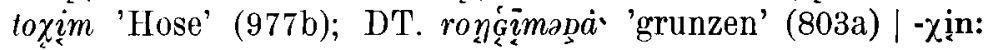

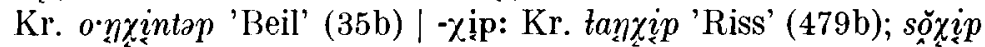

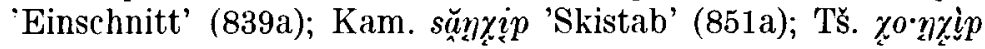

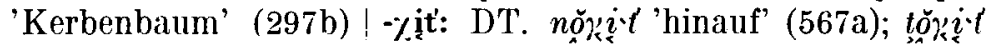
'dorthin' (975b); Kr. náă

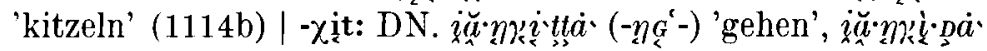

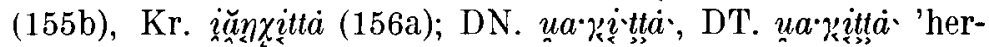
beirufen' (216b); DN. xon)ipam (i), Kr. xonxitta '[herumklet-

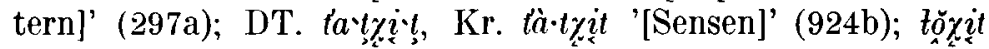

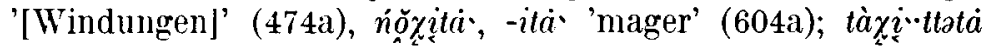
'zerreissen' (988a).

-

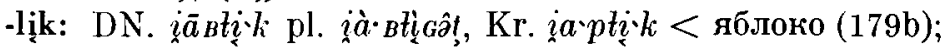

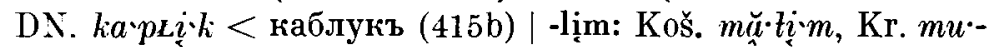

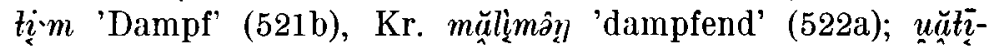
mata 'aufblicken' (224b) | -lip: Kr. $\breve{a} \cdot m t i c \cdot p$ 'Wuhne' (48a) |-lįt:

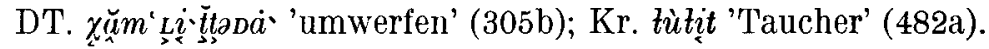

-lịn: DN. svali ‘na 'Lauf (der Flinte)' (894b).

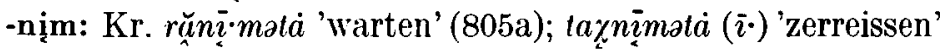

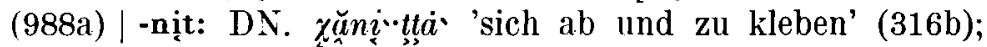

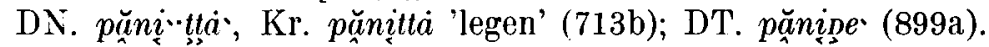
-pị: DN. sțobi 'jospa' (854b; 966b) |-piux: Kr. numpīuz, -īus 'von oberhalb' (568b) | -pịt: Kr. tă ·pịt '[Späne]' (1007a).

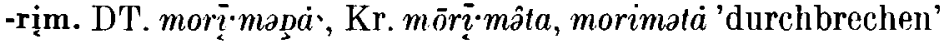

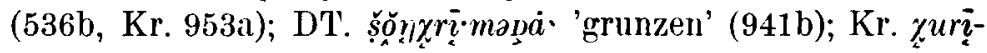

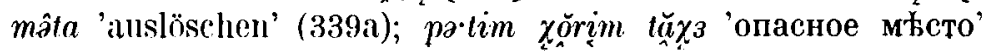


Tabelle

\begin{tabular}{|c|c|c|c|c|c|c|}
\hline \multirow[b]{3}{*}{$\begin{array}{l}\text { A) } \\
-o i\end{array}$} & \multirow{2}{*}{ DN. } & \multirow{2}{*}{ DT. } & \multicolumn{4}{|c|}{ I r tys h } \\
\hline & & & Ts. & Koš. & Fil. & Sogom \\
\hline & $\begin{array}{l}\text { x̧oi } \\
\text { soi }\end{array}$ & & $\begin{array}{l}(\chi u i) \\
\text { soi }\end{array}$ & 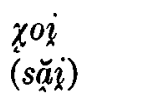 & $\chi \grave{o} i$ & $(\chi u i)$ \\
\hline $\begin{array}{l}\text { B) } \\
-o i^{-}\end{array}$ & 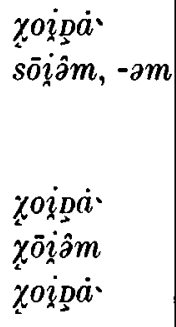 & & $\begin{array}{l}\text { sōizam } \\
\text { sōizap } \\
\chi \chi \bar{o} \not \partial a m\end{array}$ & & $\begin{array}{l}\text { sōiam } \\
\text { xōiam }\end{array}$ & 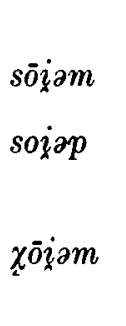 \\
\hline 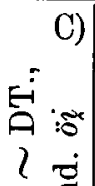 & $\begin{array}{l}\text { moi } \\
\text { poi } \\
\text { poi }\end{array}$ & $\begin{array}{l}m o i \\
p o i \\
p o i \\
p a i\end{array}$ & $\begin{array}{l}\operatorname{moi} i^{1} \\
p o i\end{array}$ & poi & $\begin{array}{l}\text { moi } \\
\text { poi }\end{array}$ & $\begin{array}{l}m o i \\
\text { poi }\end{array}$ \\
\hline $\begin{array}{ll}z_{a}^{-1} & \\
& \\
D)\end{array}$ & $\begin{array}{l}u \bar{o} i_{3} \\
\text { mōizpts } 2 \\
\text { moipe.k }\end{array}$ & 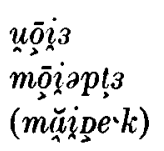 & $\begin{array}{l}u \overline{o ̄ i z} \\
(m \bar{u} i \partial p t 3)\end{array}$ & $\begin{array}{l}\text { uōiz } \\
\text { mọjapts } \\
\text { (måztèk) }\end{array}$ & $\begin{array}{l}\text { (mūįapls) } \\
\text { moịpèk }{ }^{3}\end{array}$ & $\begin{array}{l}u \underline{p o ̄ i 3} \\
\text { moìtèk }\end{array}$ \\
\hline$-o \dot{n}-$ & & & 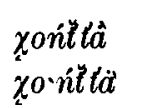 & & & \\
\hline$-o t-$ & sòtt'á & & & & & \\
\hline
\end{tabular}

1 Aber Ts. moi $(871 \mathrm{~b}, 1068 \mathrm{a})$.

2 Aber im KT: DN. mōitapț, mōîapț (500a).

3 Auch moingèk (501a).

4 Auch sōiap (820b). 
XVI

\begin{tabular}{|c|c|c|c|c|c|c|c|}
\hline \multicolumn{4}{|c|}{ Kon d a } & \multirow{2}{*}{ Bedeutung } & \multicolumn{3}{|c|}{ Belegstellen } \\
\hline (OL) & $\mathrm{Kr}$. & Kam. & (PD) & & $\mathrm{OL}$ & PD & $\mathrm{KT}$ \\
\hline $\begin{array}{l}\chi 0 / u i \\
\text { soi }\end{array}$ & $\begin{array}{l}(\chi u i) \\
\text { soi }\end{array}$ & TS. $\chi 0 i$ & $\begin{array}{l}(\chi \dot{u} i \dot{i}) \\
\text { sòi } \\
\operatorname{sò} i-\chi o n \bar{a} \cdot \dot{a} a \eta\end{array}$ & $\begin{array}{l}\text { (Ehe)mann } \\
\text { anas clangula } \\
\text { [rötlich . . . hell] }\end{array}$ & $\begin{array}{c}99 \\
88,94\end{array}$ & $\begin{array}{r}548 \\
2206 \\
2207\end{array}$ & $\begin{array}{l}279 a \\
817 a\end{array}$ \\
\hline $\begin{array}{l}\text { xoi- } \\
\text { sōįam } \\
\text { sōiop }\end{array}$ & 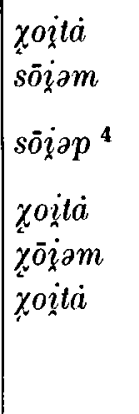 & tŞōọiat & 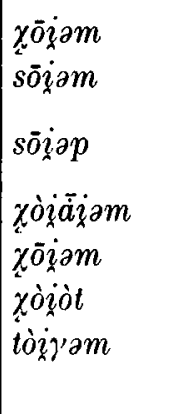 & $\begin{array}{l}\text { treffen } \\
\text { Bach } \\
\text { Netzgewebe } \\
\text { [Bärenschlund] } \\
\text { welken } \\
\text { Asche } \\
\text { kosten } \\
\text { schwingen } \\
<\text { syrj. Geld }\end{array}$ & $\begin{array}{l}94 \\
94 \\
94 \\
- \\
- \\
- \\
- \\
-\end{array}$ & $\begin{array}{r}479 \\
2209 \\
2211 \\
2210 \\
476 \\
478 \\
481 \\
2566 \\
-\end{array}$ & $\begin{array}{l}281 \mathrm{a} \\
819 \mathrm{~b} \\
820 \mathrm{~b} \\
283 \mathrm{~b} \\
281 \mathrm{~b} \\
283 \mathrm{~b} \\
- \\
925 \mathrm{a}\end{array}$ \\
\hline 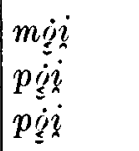 & $\begin{array}{l}m \dot{q} i \\
p \dot{q} i \\
p \dot{q} i \\
p \dot{q} i\end{array}$ & & $m \dot{m o i}$ & $\begin{array}{l}\text { Hochzeit } \\
\text { Espe } \\
\text { Schutzbrett }\end{array}$ & $\begin{array}{l}94 \\
94 \\
94\end{array}$ & $\begin{array}{r}1275 \\
1897 \\
-\end{array}$ & $\begin{array}{l}499 \mathrm{a} \\
658 \mathrm{a} \\
657 \mathrm{~b}\end{array}$ \\
\hline 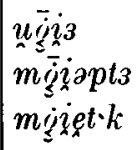 & 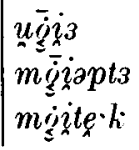 & Tร̌. $\operatorname{uop}_{n} i 3$ & $\begin{array}{l}\text { wöìz } \\
\text { mòitèk }\end{array}$ & $\begin{array}{l}\text { Tier } \\
\text { Rätsel } \\
<\text { syrj. Seife }\end{array}$ & $\begin{array}{r}94 \\
107 \\
94\end{array}$ & $\begin{array}{l}3059 \\
1276\end{array}$ & $\begin{array}{l}204 \mathrm{~b} \\
500 \mathrm{a} \\
501 \mathrm{a}\end{array}$ \\
\hline xa'ñt- & & 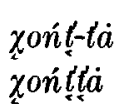 & 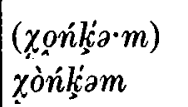 & $\begin{array}{l}\text { krank sein } \\
\text { abschälen }\end{array}$ & 88,90 & $\begin{array}{l}504 \\
505\end{array}$ & $\begin{array}{l}322 \mathrm{~b} \\
322 \mathrm{~b}\end{array}$ \\
\hline & soțta & & $s \bar{k} k \underline{\prime} \partial \cdot m$ & fangen (Ball) & - & 2225 & $883 b$ \\
\hline
\end{tabular}


Tabelle

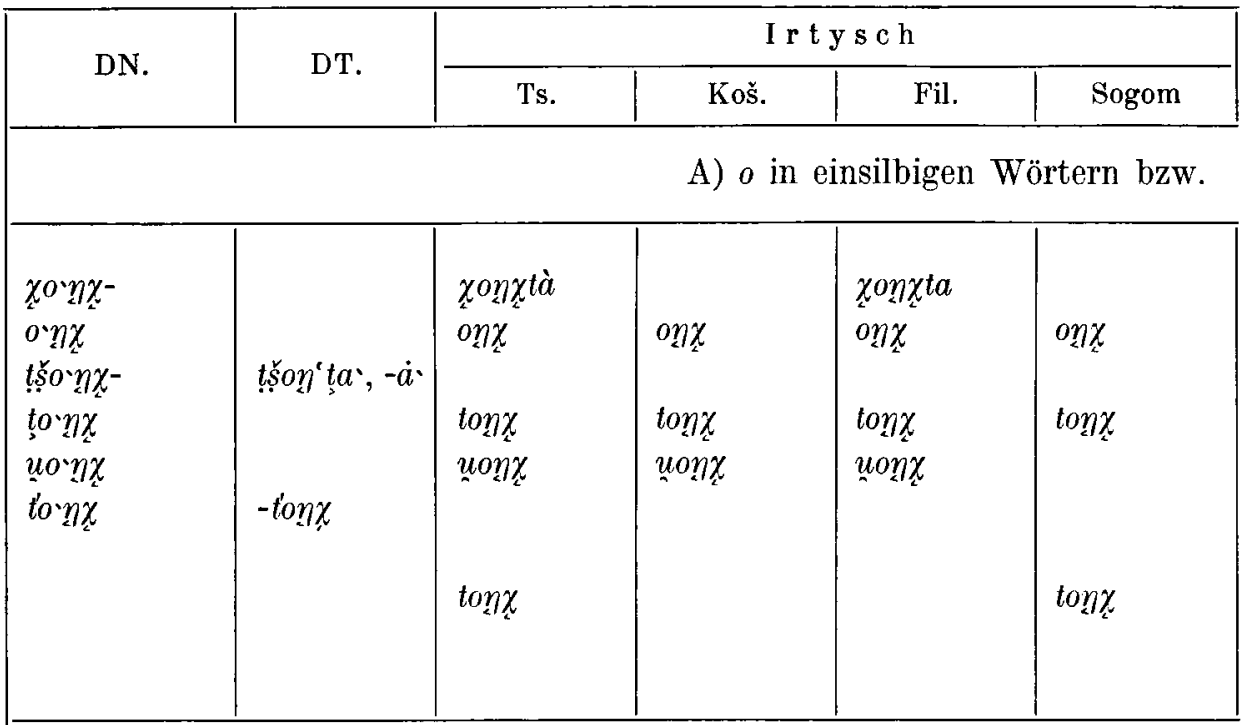

B) 0 in mehrsilbigen

\begin{tabular}{|c|c|c|c|}
\hline 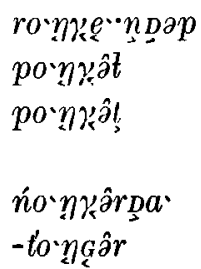 & po $\eta \chi_{r} \partial \hat{t}$ & 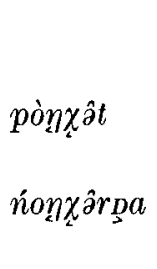 & pòy $\chi^{\partial \hat{t} t}$ \\
\hline
\end{tabular}

C) DN. $-o \eta-\sim \mathrm{DT}$.

\begin{tabular}{|c|c|c|}
\hline 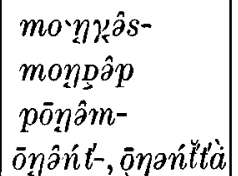 & 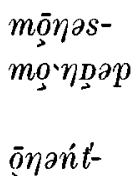 & $m o \cdot \eta \chi \partial \hat{s} s t a$ \\
\hline
\end{tabular}




\section{XVII}

\begin{tabular}{|c|c|c|c|c|c|c|c|}
\hline \multicolumn{4}{|c|}{ Kond a } & \multirow{2}{*}{ Bedeutung } & \multicolumn{3}{|c|}{ Belegstellen } \\
\hline (OL) & $\mathrm{Kr}$. & Kam. & $(\mathrm{PD})$ & & $\mathrm{OL}$ & PD & KT \\
\hline
\end{tabular}

einsilbigen Wortstämmen vor $-\eta \varkappa$

\begin{tabular}{|c|c|c|c|c|c|c|c|}
\hline po:n\% & 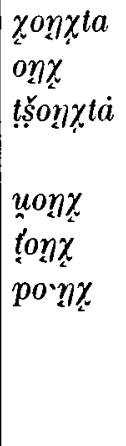 & 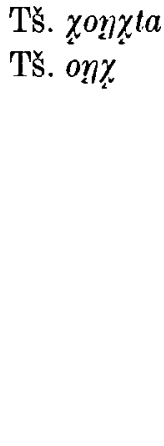 & 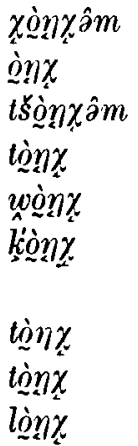 & $\begin{array}{l}\text { klettern } \\
\text { Harz } \\
\text { ausschlagen } \\
\text { Götze } \\
\text { Grube } \\
\text { Russ } \\
\text { [Netzbreite] } \\
\text { [Hufrinde] } \\
\text { [gezierte Wand] } \\
\text { Wunder }\end{array}$ & $\begin{array}{r}96 \\
96 \\
97 \\
102 \\
103 \\
105 \\
105 \\
- \\
- \\
-\end{array}$ & $\begin{array}{r}506 \\
1547 \\
2769 \\
2600 \\
3035 \\
964 \\
- \\
2598 \\
2599 \\
1034\end{array}$ & $\begin{array}{c}296 \mathrm{~b} \\
35 \mathrm{a} \\
941 \mathrm{~b} \\
1065 \mathrm{a} \\
218 \mathrm{a} \\
904 \mathrm{~b} \\
689 \mathrm{a} \\
1066 \mathrm{a} \\
-- \\
-\end{array}$ \\
\hline
\end{tabular}

Wörtern vor $-\eta \eta$

\begin{tabular}{|c|c|c|c|c|c|c|c|}
\hline 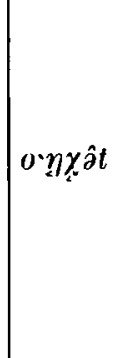 & 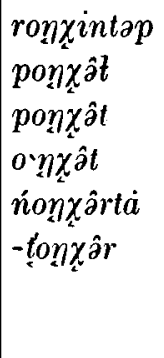 & ronxe:ntap & 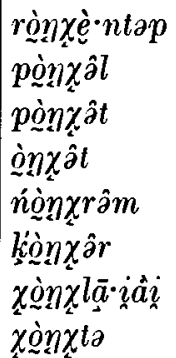 & $\begin{array}{l}\text { Glocke } \\
\text { [Baumknollen] } \\
\text { Geschwulst } \\
\text { [Bärenkopf] } \\
\text { nagen } \\
\text { Wasserjungfer } \\
\text { [dreingrimmen] } \\
\text { [stromaufwärts] }\end{array}$ & $\begin{array}{r}97 \\
100 \\
100 \\
99 \\
- \\
- \\
- \\
-\end{array}$ & $\begin{array}{r}2021 \\
1849 \\
1850 \\
1548 \\
1498 \\
965 \\
507 \\
508\end{array}$ & $\begin{array}{r}803 \mathrm{a} \\
691 \mathrm{a} \\
692 \mathrm{a} \\
38 \mathrm{a} \\
614 \mathrm{a} \\
906 \mathrm{~b} \\
-\end{array}$ \\
\hline
\end{tabular}

$-o \eta-\sim$ Kond. $-\ddot{o} \eta-(\sim o \eta \chi x)$

\begin{tabular}{|c|c|c|c|c|c|c|}
\hline $\begin{array}{l}m o \cdot \eta x^{2} s- \\
m \dot{q} \cdot \eta t \partial p \\
p \dot{q} \cdot \eta m \partial \underline{s}-\end{array}$ & 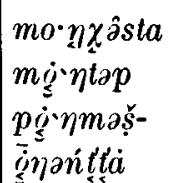 & 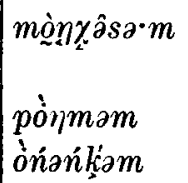 & $\begin{array}{l}\text { abwischen } \\
\text { Waschbündel } \\
\text { Zeit haben zu . } \\
\text { [schlucken] }\end{array}$ & $\begin{array}{r}98 \\
98 \\
108 \\
109\end{array}$ & $\begin{array}{l}1250 \\
1910 \\
1579\end{array}$ & $\begin{array}{r}519 \mathrm{~b} \\
519 \mathrm{~b} \\
686 \mathrm{~b} \\
33 \mathrm{a}\end{array}$ \\
\hline
\end{tabular}


(778a); tă rịm, -im 'verdorben' (921b) | -rip: Kr. nóŏxịpp 'Messer' (606b) |-rįt: DN. xŏrị๋t '[Lockvögel]' (331a); Kr. tò‘rịt '[Kraniche]' (1013a).

-sịp: Kr. ńăxsịp, -i`p 'Messer zum Schuppen' (608b)|-sịt: Kr. uă

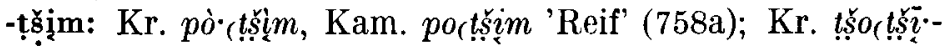
mâta, -imata 'weggiessen' (962a).

-tịu: DN. nŏrtivw' '. . Stange' (595b) |-tịm: Kr. uăttịmtà 'fremd, unbeteiligt' (270b); Kr. xă

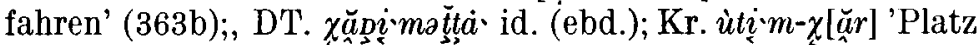

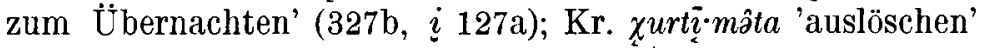
(339a); DT. ńŏD̃ $\vec{\imath} \cdot m \partial \underline{a} \dot{a}$ 'schnell ablecken' (650b) | -tịn: DT.

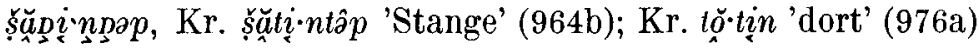

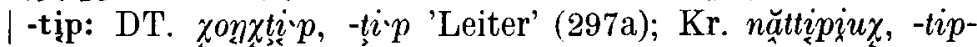
'Tragstange' (599b); poxxtip, Kam. poxtįp 'Fischgabel' (677b) | -tịt: DN. ă

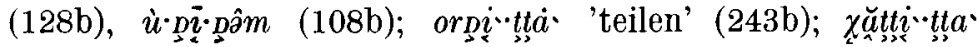

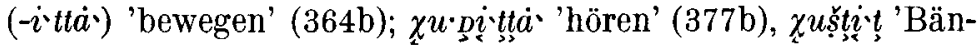

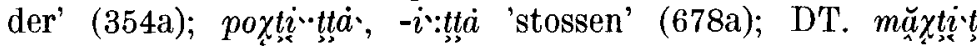

Tabelle XVIII

Konsonantenumgebung von DN., DT. $。$ in der ersten Silbe ${ }^{1}$

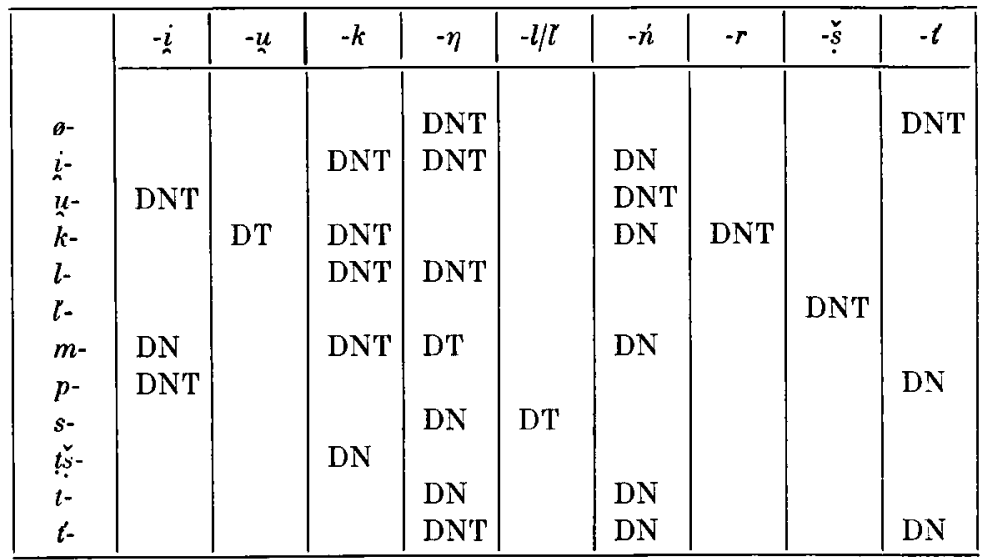

1 DNT = belegt in DN., DT.

$\mathrm{DN}=" \Rightarrow \mathrm{DN}$.

$\mathrm{DT}="$ T $\mathrm{DT}$. 
Tabelle XIX

Konsonanten um $g(j)$ in der ersten Silbe am Irtysch ${ }^{1}$

\begin{tabular}{|c|c|c|c|c|c|c|c|c|}
\hline & -0 & $-i$ & $-k$ & $-\eta$ & $-\dot{n}$ & $-p$ & $-r$ & $-t$ \\
\hline o- & \multirow{9}{*}{ TsP } & \multirow{9}{*}{ FKTs } & \multirow{9}{*}{ TsSg } & \multirow{9}{*}{$\begin{array}{l}\text { FKSgTs } \\
\text { F }\end{array}$} & & & \multirow{9}{*}{ FKSgTs } & \multirow[t]{4}{*}{$\mathrm{FSgT}$} \\
\hline !- & & & & & & & & \\
\hline$\frac{2-}{2}$ & & & & & Is & & & \\
\hline$m$ & & & & & Ts & & & \\
\hline$p-$ & & & & & & & & Ts \\
\hline$s=$ & & & & & & & & \\
\hline$t$ & & & & & & & & \\
\hline$t=$ & & & & & KSgSvTs & & & Sv'Ts \\
\hline$t^{\prime} s^{-}$ & & & & & & Ts & & \\
\hline
\end{tabular}

$1 \mathrm{~F} \quad=$ belegt in Fil.

FKSgTs = "Fil., Kos̀., Sogom, Ts.

FKTs $="$ "Fil., Koš., Ts.

FSg = "Fil., Sogom

FSgTs $="$ "Fil., Sogom, Ts.

FTs $=$ "Fil., Ts.

KSgSvTs $=$ "Koš., Sogom, Sav., Ts.

SvTs $="$ "Sav., Ts.

Ts $=$ "T's.

$\mathrm{TsP} \quad="$ "Ts. (PD)

TsSg $="$ "Ts., Sogom

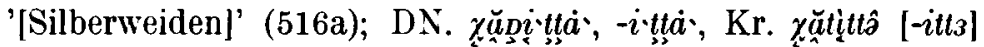

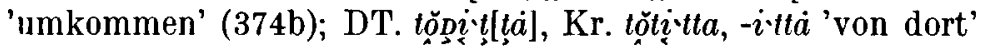

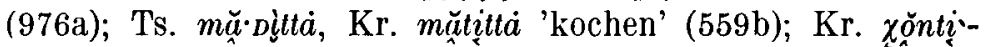
tta, -ittti' ... umherlaufen' (320a); Kr. tă ptịt '[Seerosen]' (490b).

Belegstellen zu den Tabellen XVIII-XX

S. OL $70-77$, oben S. 18-26, ausserdem (nur die Belegstellen - in Stichwörtern und in Beispielsätzen - werden angegeben):

$\ddot{o w} w$-: Kr. 15a, 18a | $\ddot{k} k-:$ Kr. 30b, PD 1570-1; | $0 \eta-:$ DN., DT., Fil, Ts., Ť., Kr. 32b; DN., Koš., Fil, Ts., Sogom, Tš., Kr. 34a; 


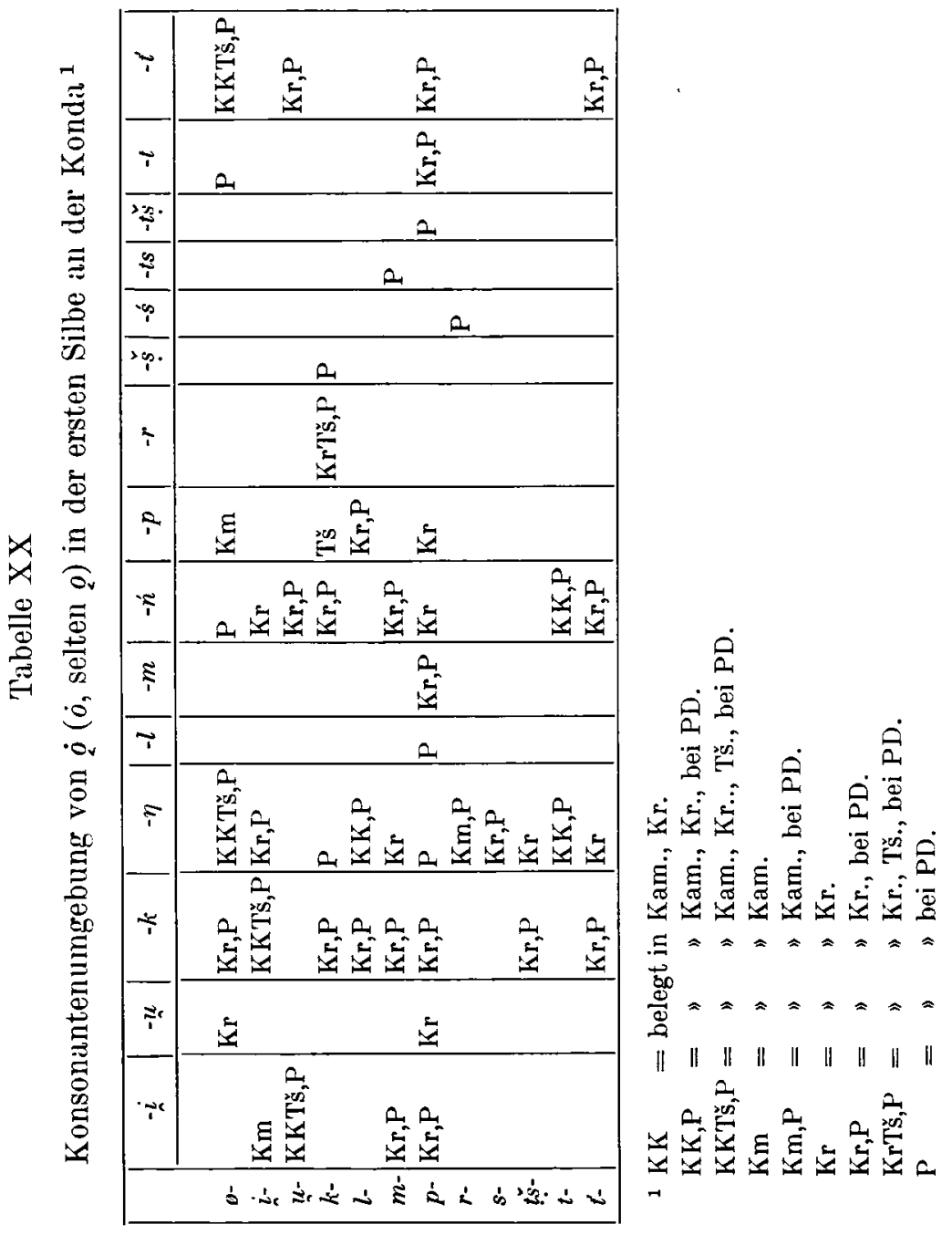


DN., Ts., Sogom, Kr. 40ab; DN., Ts., Kr., Kam. 34b; DN., Kr. 40a; DN., Koš., Ts. 359b; DN., Kr. 33a, 34a, b, 40a, 334a, 980a; DN. 32b, 91a, 284a, 316a, 658b, 708b, 741a, 763a, 860a, 900a, 916b, 983a, 1009a; Kr. 400b; PD 1580-5| ön-: PD 1579 | $\ddot{p} p-:$ Kam. 20b, | $t^{\prime-}:$ DN., Fil, Ts., Sogom, Tš., Kr., Kam. 92ab; DN., DT., Kr. 32b; DN., Kr., Kam. 131b; Sogom, Kr. 93a; DN., Kr. 29b; Kr. 96a, 133a, 871b, 924a, 1028a; PD 1573-6; | öt-: PD 1587.

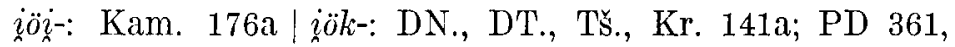
$364 \mid$ iör $-:$ DN., DT., Kr. 80a, DN., Fil., Kr. 156b, DN., DT. 266b, 404b, 647b; DN., Kr. 401a, 1019b, DN. 9b, 111b, 361a, 371a, 436a, 522b, 649a, 1082b, Kr. 497b, 1043b, PD 365 | iön-: DN., Kr. 176a.

ü̈i-: s. oben, S. 19-20, DN., Kr. 12b; PD 3058-60| иön-: DN., DT., Kr. 232a (DN. o), DN. 71a, 527b, Ts. 795b; PD 3062-3|ütt: Kr. 253ab; PD 3061.

köu-: DT. 389b | kök-: DN., DT., Kr. 49b, 391a, DT. 214a, Kr. 354b, PD 812 | kö -: PD 838 | kön-: DN., Fil., Ts., Kr. 413b, PD 835 | köp-: Tš. 414b | kör(-): DN., DT., Koš., Fil, Sogom; Tš., Ts., Kr. 416b-7a; DN., Ts. 130a, DN., Kr. 433b, 764a, DN. 75a, 623b, 868a, 934b, 1038b, DT. 865a, Ts. 129b, Kr. 209a, 794a, 904a; DN., Fil., Kr. 429a; DN., Ts., Kr. 432b; Ts. 432a, 813a; PD 842-3, 848, $850 \mid$ köš- PD 858.

lök(-): DT., Kr. 471a; 211a, DT., Kr. 471b;475b; DN. 472b, $473 \mathrm{a}, 136 \mathrm{~b}, 140 \mathrm{a}, 144 \mathrm{~b}, 150 \mathrm{a}, 218 \mathrm{~b}, 459 \mathrm{a}, 488 \mathrm{a}, 660 \mathrm{~b}, 722 \mathrm{~b}$, $728 \mathrm{a}, 739 \mathrm{a}, 1031 \mathrm{a}, ? 127 \mathrm{~b}, 155 \mathrm{~b}, \mathrm{DT} .79 \mathrm{~b}, 475 \mathrm{~b}, 477 \mathrm{~b}, 1018 \mathrm{~b}$; Kr. 98b, 475b, 476a, 838a, 902b, PD lök(-): 1076--7, 1080-1। lö $\eta$-: DN., DT., Kr. 480b, Kr., Kam. 480a, Kr. 805b, PD 1082 | -l/löp-: Kr. 469a, PD $778 \mid$-l’öss: DN., D'T. 383b.

mö̈(-): s. oben, S. 20, DN. 58a, 737a, Ts. 871b, 1068a, DN. $(\sim 0)$, Koš., Kr. 500a, DT., Kr. 499a; DN. $(\sim 0)$ 499b; DN. 500a, Kr. 500ab, 583a; Fil. $(\sim 0)$, Kr. 501a; PD 1275-6| mök-:

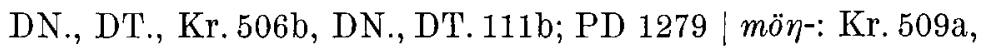
DT., Kr. 519b | möń-: DN., Kr. 531ab, Ts., Kr. 531b, 886a, Ts. 497b ( o 531a), PD $1280 \mid$ möts-: PD 1284-5.

pöi-: DT., Kr. 657b, 658a; DN. ( o 657b), DN., Kr. 813a; DN., Kr. 660b; PD 1897-1900, 1866 | pöu-: Kr. 663a, 978b pök-: Kr. 670a, PD 1903 | pöq-: PD $1910 \mid$ pöl-: PD $1907 \mid$ 
pöm-: Kr. 704b; PD 1908 | pöni-: Kr. 716b | pöв-: Kr. 718b | pötš-: PD 1913 | pöt-: DN., Kr. 746b, 1)N. 325b, 459a, 1024b; Ts. ( o 746b) 639a, Kr. 163a, 1018b; PD 1906 |pöt-: Kr. 814a, PD 1911-2.

röij-: Kam. 802b, PD 2029 | röś-: PD 2031.

sö· Ts.: PD 2256 |söı(-): DN., Ts., Kr. 848a, DN., Kr. 1030b, DN. 52a, 344b, Kr. 847b; PD 2255 | sölll'-: DT. 854ab. tšök-: DN., Ts., Sog., Kr. 929b-30a, DN. 585b, 586a, 1028b, T's. 534a, Kr. 679b; PD 2776, 2778, $2781 \mid$ tšön-: Kr. 940a.

tö $\eta$-: DN., Kr., Kam. 993a, Kr. 991b; PD 2639 | tön s. oben, S. 24, PD 2634-5.

tök-: Kr. 899a, PD 969 |-tör-: DN., DT., Kr. 455b, DN., DT. 266b, 361a, 371a, 647b, DT., Kr. 9b, DN. 1031a; tönSav. 50a s. oben S. 23-4, PD 973-5|töt:- s. oben S. 24, PD 970-1 || t's̈ö $B^{-}$: Ts. $233 \mathrm{~h}$.

\section{Belegstellen zu den Tabellen XXI-XXIII}

S. OL 78-87, oben S. 26-31, ausserdem (nur die Belegstellen - in Stichwörtern und in Beispielsätzen - werden angegeben):

ŏu(-): Kr., Kam. 20a; Kam. 15a, PD 1589| ök-: PD 1572 |

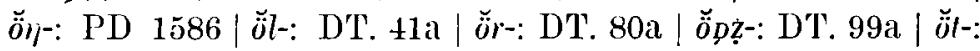
PD 1588 | ŏt'-: DN., D'T. 133b, PD 1578.

i DT. $187 \mathrm{~b}||$ ü̆ $\gamma-:$ DN. $217 \mathrm{ab}$.

kö̆-: DN. 383a, 384a, 385a; 410b, 493a, Kr. 980a; PD 800-2 | kö̆u-: DN., DT. 387ab, DN. 160b, DT. 387a; PD $869 \mid k \ddot{o ̈ k}(\gamma \mid-)$ : DN., DT., Kr. 29b; DN., Kr. 391b, Kr. 391b; PD 813-5 । kö̈n-: Ts., Kr. 448b; PD 836-7| köll(l')-: DN., Kr. 397a, Ts. 399b, Kr. 397ab, 400a, 779a, 966a; PD 820-3 | kô̆m-: DT., Koš., Ts., Kr. 404a, DN., Kr. 406a, DN. 403a, DT. 404a; PD 824-31 kö̆n-: DN., DT., Koš., Fil., Ts., Sogom, Tš., Kr. 408a, DN. 475a, 945b, Ts. 412b; PD 832-4|kön-: DT. 413b|köp-: DT., Kr. 414b; Koš. 760a ( $\sim 413 \mathrm{~b})$; DT., Kr. 414b, Kr. 413b, 414b, Kam. 414a; PD 839-41|kör(-): DN., DT., Ts., Kr., Kam. 418b-420b; DN., DT., Kr. 420b, DN., 29b; DN., Kr. 423b; DN., Ts., Kr. 424a; 426ab; Ts., Kr., Kam. 435b; 


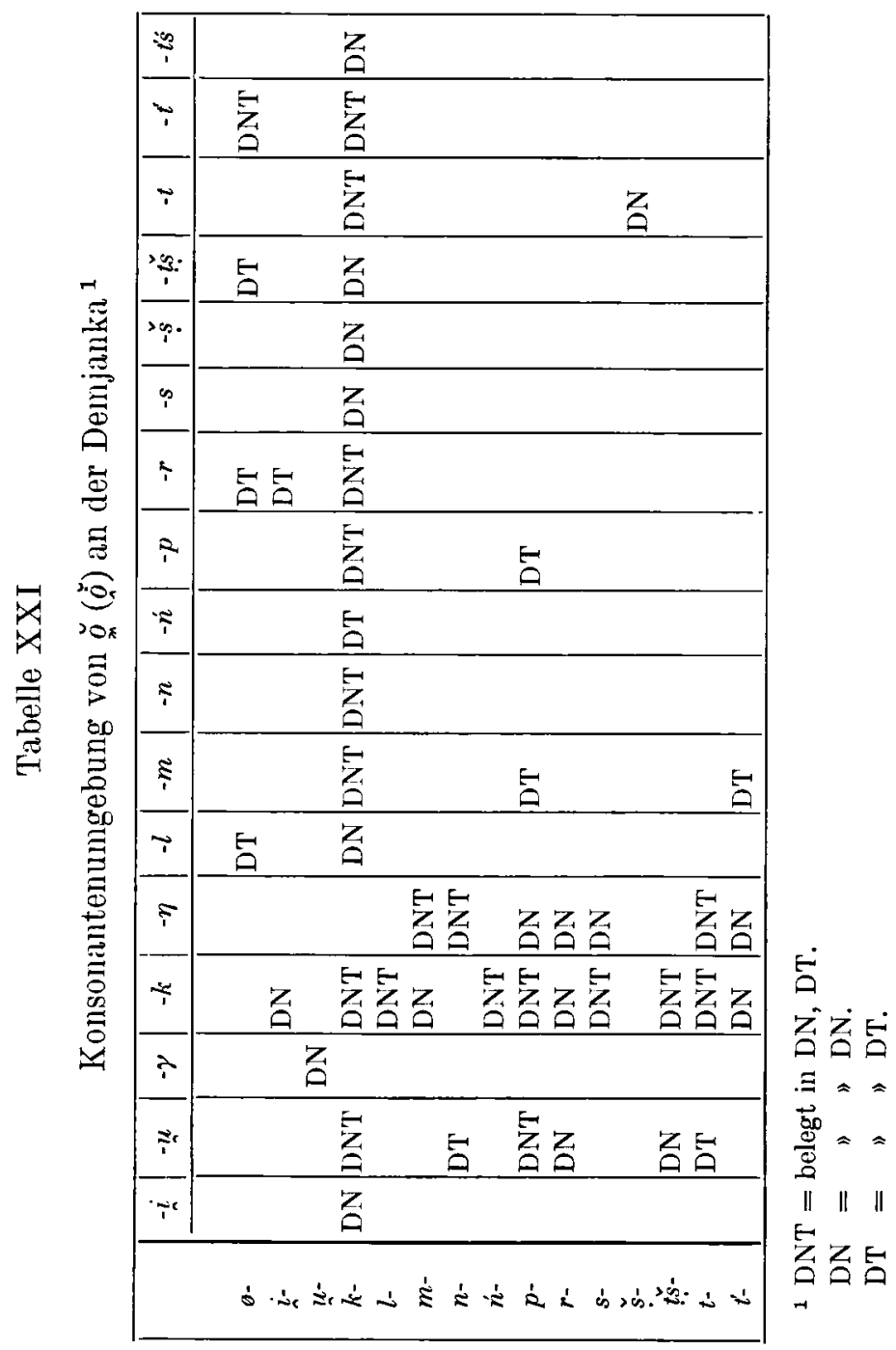


Tabelle XXII

Konsonantenumgebung von $\underset{x}{\circ}$ am Irtysch $^{1}$

\begin{tabular}{|c|c|c|c|c|c|c|c|c|c|c|c|}
\hline & $-k$ & $-\eta$ & $-l$ & $-m$ & $-n$ & $-p$ & $-r$ & $-\stackrel{s}{s}$ & $-\underline{t}$ & $-t$ & $-t$ \\
\hline$\frac{i}{k}$ & $\mathrm{Ts}$ & Ts & Ts & KTs & FKSgTs & K & SgTs & FK & Ts & Ts & KTs \\
\hline$\dot{n}-$ & Ts & & & & & & & & & & \\
\hline$p-$ & & $\mathrm{K}$ & & & & & & & & & \\
\hline$t-$ & $\mathrm{Ts}$ & $\mathrm{Sv}$ & & & & & & & & & \\
\hline$t-$ & Ts & & & & & & & & & & \\
\hline
\end{tabular}

1 FK = belegt in Fili., Koš.

FKSgTs = " Fili., Koš., Sogom, T's.

$\mathrm{K}="$ "Koš.

KTs $=$ " Koš., Ts.

SgTs $="$ Sogom, Ts.

$\mathrm{Sv}="$ "Sav.

Ts $="$ "Ts.

DN., DT. 870a, 943b; DN., Sogom923b; DN., Kr. 423b, 426a, $433 \mathrm{ab}, 685 \mathrm{~b}, 745 \mathrm{a}, 764 \mathrm{a}, 867 \mathrm{~b} ;$ DN. 7b, 29b, 140a, 196a, 253a, 254b, 293a, 296b, 415a, 424a, 460b, 593b, 631a, 714b, 721b, 728a, 864b, 982a, 1084a, DT. 697a; Ts. 158b, 780a; Kr. 361a, 433a, 435b, 455b, 712a, 752a, 974b; PD 844-7, 853-5 | kŏss-: DN., Kr., Kamı. 436b; PD 803, 805, 856|köš(-): DN., Koš., Fil., Tš. 448b; DN., Kr. 450a; Kr., Kam. 447b; DN.

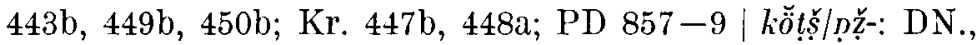
Ts., Kr. 450a; DN., Kr. 447b, Kr. 447a, 449b, 450b, PD 865-8| kốt(-): DN., DT., Ts., Kr. 452a; DN., Ts., Kr., Kam. 461b; DN., Ts., Kr. 450b-1a, 144b, 685b; DN., DT., 451b; DN., Kr. 456b; DN. 100b, 108a, 135b, 214a, 255a, 356b, 636b; 692a, 855a, 931a, 971a, 1023b, 1070a; Ts. 1039a; Kr. 141b, $454 \mathrm{~b}, 712 \mathrm{a}$; PD 806-11, $860 \mid k o ̈ t^{\prime}(-):$ DN., Koš., Ts., Kr., Kam. 441ab; DN., DT., Ts., Kr. 440ab; DN., Kr. 441b-2a, 468a; DT., Kam. 467b, DN. 968a, 1054b; Salym 218a; DT. 440b, Kam. 441a; PD 816-9, 862 | kô̆t's/b́ź-: DN., Kr., Kam. $441 \mathrm{~b}, \mathrm{DN} .759 \mathrm{a}$.

l/rök(-): DN., D'T., Kr., Kam. 477a; DN., Kr. 474b; DN. 


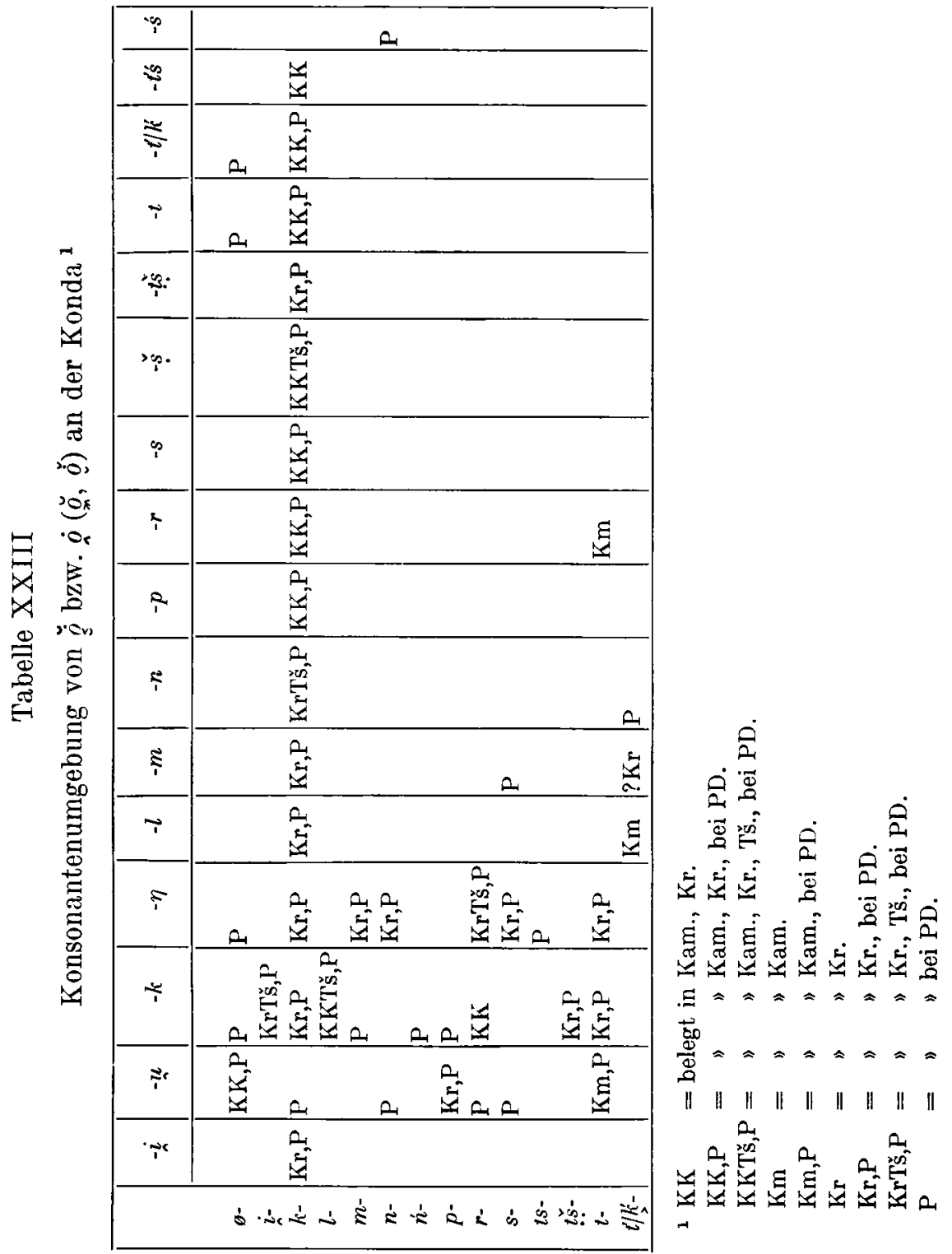


90a, 384b, 475a, 490a, 966a; Tš., Kr. 682b; Kr. 67b; Kam. 479a; PD 1078-9, in der zweiten Silbe 2892.

$m \check{o ̈ k} / G-:$ DN. 509b; PD $1277 \mid m \ddot{o n}(-)$ : DN. (58a, 100a, 385at usw.), DT., Kr. MSFOu 128: 37, 75, PD 38, 484 usw. 1282.

nö̀u-: DT. 565b, PD 1382-4|nönl(-): DN., Kr. 469a; IN. 58a, 167b, 287a, 469a usw. D'., Kr. MSFOu 128: 38, 75, Kr. 449b, PD 712, 1380 usw. | nŏśs- PD 1381.

ńö̌k/G-: DN., DT., Ts. 604a-5a; PD 1505.

pö́u-: DN., DT., Kr. 661a, 662a; DN., Kr. 661b-2a, D'T. 661b; PD 1914--20|pök-: DN., D'T. 666a; DN. 669b, 834b; PD 1901-2, 1904- $5 \mid$ pö̀-: DN., Koš. 687a, DN. 41a, 282b, 813a pö̈m(-): DT. 703b. pöp-: D'T. (OL 85).

rŏ́u-: DN. 839a; PD 2032-4|rŏk-: DN., Kr., Kam. 795a | rö̆ı-: DN., TŠ., Kr. 803b; PD 2030.

ső́w(-): $\mathrm{PD}$ 2257-9|sŏk(-): DN. 833b; DT. 839b|sŏ̋(-): $\mathrm{DN}$., Kr. 846b, 848a, DN. 850b, 5̄55a; PD 2254|sŏm-: $\mathrm{PD}$ 2252 .

šŏt-: DN. 964a.

tsö̈r-: PD 2716.

țş̌ôn-: DN. 928a | țșōk/G-: DN. 930b-1a, DT., Kr. 902b; Kr. 931a; PD 2777, 2779.

tŏıc-: DT. 466a; Kam. 972b; PD 2648 usw., 2649-55 tök-: D., DT., Ts., Kr. 1061b; Kr. 986b; PD 2633 |tö̆ $-:$ DN., DT., Kr. 990b-1a; 1063b-4a, 1070b; D., Sav. 991a; DN., DT. 1070ab, DN., Kr. 995a, 1062b, 1063a DN., Kr., Kam., 1066b; DN. 246a, Kr. 86a, 994a; PD 2636-8, 2640-7|tör-: Kam. 1039a.

tö̌k/G-: DN., Ts. 902b; DN. 901a | tô̆ $-:$ DN. 900b | kุŏ́l-: Kam. 907a | tô̆m-: DT. ?? Kr. 911a|tô̆n-: PD 972.

Edith Vértes 